University of Tennessee Health Science Center UTHSC Digital Commons

\title{
Development and Evaluation of Therapeutics in the Setting of Radiation Injury
}

\author{
Karin Emmons Thompson \\ University of Tennessee Health Science Center
}

Follow this and additional works at: https://dc.uthsc.edu/dissertations

Part of the Medicinal and Pharmaceutical Chemistry Commons, Natural Products Chemistry and Pharmacognosy Commons, and the Pharmaceutics and Drug Design Commons

\section{Recommended Citation}

Thompson, Karin Emmons, "Development and Evaluation of Therapeutics in the Setting of Radiation Injury" (2010). Theses and Dissertations (ETD). Paper 257. http://dx.doi.org/10.21007/ etd.cghs.2010.0316.

This Dissertation is brought to you for free and open access by the College of Graduate Health Sciences at UTHSC Digital Commons. It has been accepted for inclusion in Theses and Dissertations (ETD) by an authorized administrator of UTHSC Digital Commons. For more information, please contact jwelch30@uthsc.edu. 


\title{
Development and Evaluation of Therapeutics in the Setting of Radiation Injury
}

\begin{abstract}
The possibility of an incident involving radiation exposure is at the forefront of public attention in the current global environment. With a greater number of entities possessing nuclear weapons and increased demand for nuclear power, the concern for uncontained exposure to radiation is increasing. In light of these developments, the need for treatments that may be easily stored and administered to large numbers of people who have been exposed to high doses of total body irradiation (TBI) is more urgent than ever before.
\end{abstract}

Our goal was to identify and develop novel, drug-like small molecules that would exhibit radiomitigating activity for use in Acute Radiation Syndrome (ARS). Hot water extracts of Cat's Claw containing quinic acid have been shown to possess activity in increasing circulating white blood cells as well as promoting DNA repair. Our group previously synthesized and evaluated a series of quinic acid derivatives. Quinic acid derivative KZ-41 was identified and described as highly water soluble and orally available. We have, therefore, identified a novel quinic acid derivative, KZ-41, as our initial lead molecule for evaluation, characterization, and development in the setting of ARS.

Initial efficacy was tested in vivo by evaluating the impact KZ-41 had on the primary endpoint of mortality in lethally irradiated C57BL/ 6 mice 30 days after exposure. To investigate the potential mechanisms related to the enhanced survival following TBI, we investigated the effect of KZ-41 on parameters important to initial wound healing. We developed an imaging model to specifically investigate the effect of KZ-41 on clotting following TBI and vascular injury. This model also allowed us to gain insight into "combined injury" which is also of critical importance when discussing the "real-life" implications of ARS. .

To further evaluate mechanisms of effect, we developed an in vitro model using human monocytes (U937) cells. Using the in vitro model developed, we characterized the effect KZ-41 had on intrinsic and extrinsic apoptotic cell death, focusing on the impact in the "bystander effect" model. We also utilized the in vitro model to identify potential more potent analogues and next-generation compounds. Initial preclinical pharmacokinetic characterization of a next-generation compound was also conducted.

We have shown KZ-41 to be effective in improving survival of lethally irradiated mice, enhancing the critical wound healing events of initial thrombus formation and in restoring vascular flow in combined injury. In an in vitro bystander effect model, KZ-41 can mitigate apoptosis when administered after the radiation event. Initial, identification, and pharmacokinetic characterization of a potent next-generation compound for use in ARS is also described herein.

\section{Document Type}

Dissertation

\section{Degree Name}

Doctor of Philosophy (PhD)

Program

Pharmaceutical Sciences

Research Advisor

Charles R. Yates, Pharm.D., Ph.D. 


\section{Keywords}

Acute Radiation Syndrome, HEM-ARS, Radiomitigant

\section{Subject Categories}

Medicinal and Pharmaceutical Chemistry | Medicine and Health Sciences | Natural Products Chemistry and Pharmacognosy | Pharmaceutics and Drug Design | Pharmacy and Pharmaceutical Sciences

\section{Comments}

Two year embargo expired December 2012 
DEVELOPMENT AND EVALUATION OF THERAPEUTICS IN THE SETTING OF RADIATION INJURY

\author{
A Dissertation \\ Presented for \\ The Graduate Studies Council \\ The University of Tennessee \\ Health Science Center \\ In Partial Fulfillment \\ Of the Requirements for the Degree \\ Doctor of Philosophy \\ From The University of Tennessee
}

By

Karin Emmons Thompson

December 2010 
Copyright (C) 2010 by Karin Emmons Thompson All rights reserved 
For Chris, my friend and forever love. 


\section{ACKNOWLEDGMENTS}

The task of obtaining my Ph.D. would have been insurmountable without the encouragement and support of many people. First, I would like to express deepest respect and gratitude to my mentor, Dr. Ryan Yates, for his guidance and enthusiasm for research and discovery. It was extremely rewarding to be trained under such an excellent scientist (and eternal optimist) who continuously fostered the importance of the "big picture". I would also like to thank my committee members, Dr. Duane Miller, Dr. Bernd Meibohm, Dr. Waleed Gaber, and Dr. Scott Sinclair for all of their helpful advice and suggestions.

Special thanks to my colleagues, past and present, who assisted in data generation and helpful scientific discussions including Dr. Wenlin Deng, Dr. Les Stuart, Dr. Janice Zawaski, Dr. Christy Wilson, Fei Ma, Wenbo Ge, Kui Zeng, and Chaela Presley.

I would like to express heartfelt appreciation to my parents, Alan and Donna Emmons. Thank you for starting an inquisitive young mind out with a microscope and dissection kit; and for following it up with unwavering encouragement and support. You have never doubted me, even when I have. The example of love and integrity you have set is one I strive to follow.

To Chris, my biggest supporter, all my thanks and love. Throughout the course of this graduate work, we have built our family together. Somewhere along the way, my dream became your dream. Your support and encouragement for me to pursue my goals has made the difference when things became difficult. A huge part of this belongs to you. I would not be who or where I am without you.

Thank you to all of those who helped me throughout this journey both named and unnamed.

This research was supported by The University of Tennessee College of Pharmacy, RxBio, Inc., EDLabs, Inc., and the National Institute of Allergy and Infectious Disease grant number 5R21AI080534. 


\begin{abstract}
The possibility of an incident involving radiation exposure is at the forefront of public attention in the current global environment. With a greater number of entities possessing nuclear weapons and increased demand for nuclear power, the concern for uncontained exposure to radiation is increasing. In light of these developments, the need for treatments that may be easily stored and administered to large numbers of people who have been exposed to high doses of total body irradiation (TBI) is more urgent than ever before.

Our goal was to identify and develop novel, drug-like small molecules that would exhibit radiomitigating activity for use in Acute Radiation Syndrome (ARS). Hot water extracts of Cat's Claw containing quinic acid have been shown to possess activity in increasing circulating white blood cells as well as promoting DNA repair. Our group previously synthesized and evaluated a series of quinic acid derivatives. Quinic acid derivative KZ-41 was identified and described as highly water soluble and orally available. We have, therefore, identified a novel quinic acid derivative, KZ-41, as our initial lead molecule for evaluation, characterization, and development in the setting of ARS.

Initial efficacy was tested in vivo by evaluating the impact KZ-41 had on the primary endpoint of mortality in lethally irradiated C57BL/6 mice 30 days after exposure. To investigate the potential mechanisms related to the enhanced survival following TBI, we investigated the effect of KZ-41 on parameters important to initial wound healing. We developed an imaging model to specifically investigate the effect of KZ-41 on clotting following TBI and vascular injury. This model also allowed us to gain insight into "combined injury" which is also of critical importance when discussing the "reallife" implications of ARS. .
\end{abstract}

To further evaluate mechanisms of effect, we developed an in vitro model using human monocytes (U937) cells. Using the in vitro model developed, we characterized the effect KZ-41 had on intrinsic and extrinsic apoptotic cell death, focusing on the impact in the "bystander effect" model. We also utilized the in vitro model to identify potential more potent analogues and next-generation compounds. Initial pre-clinical pharmacokinetic characterization of a next-generation compound was also conducted.

We have shown KZ-41 to be effective in improving survival of lethally irradiated mice, enhancing the critical wound healing events of initial thrombus formation, and in restoring vascular flow in combined injury. In an in vitro bystander effect model, KZ-41 can mitigate apoptosis when administered after the radiation event. Initial identification and pharmacokinetic characterization of a potent next-generation compound for use in ARS is also described herein. 


\section{TABLE OF CONTENTS}

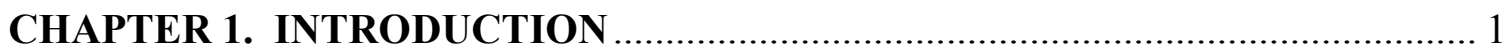

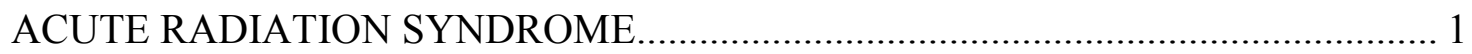

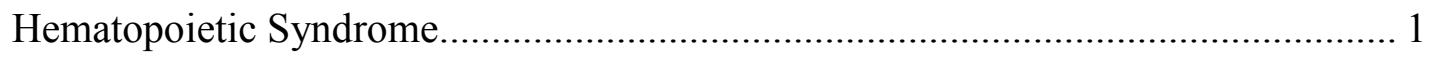

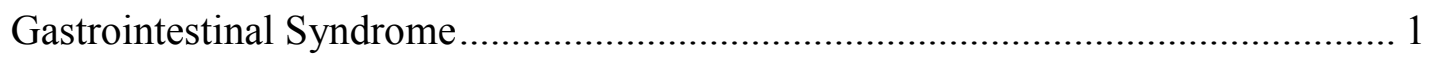

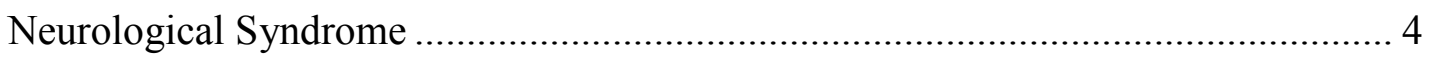

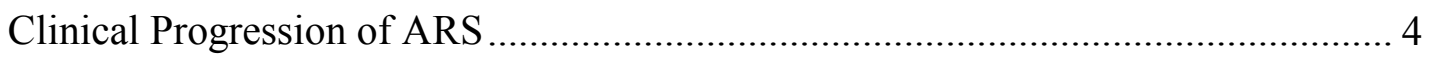

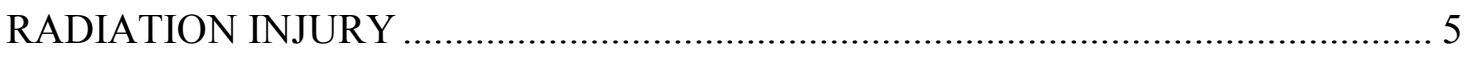

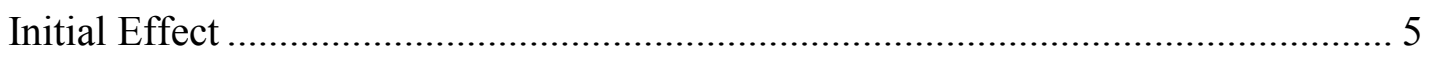

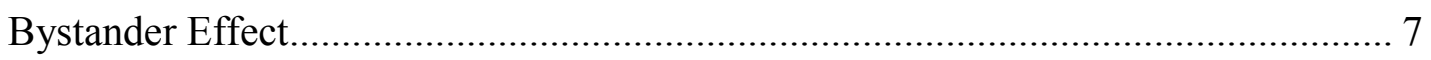

Mechanisms of Radiation-Induced Cell Death .......................................................... 7

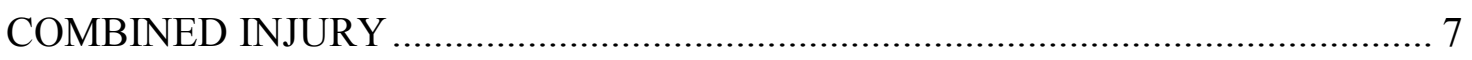

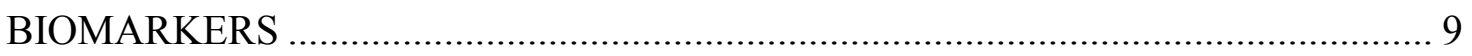

Circulating Blood Counts ............................................................................... 9

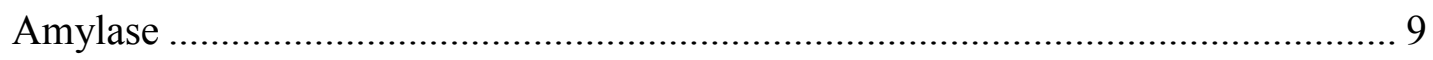

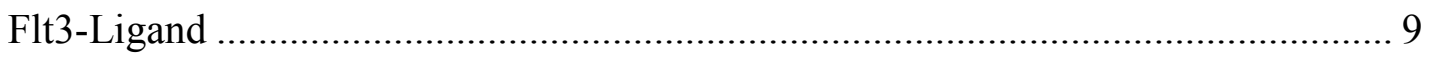

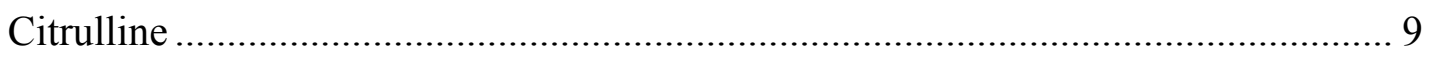

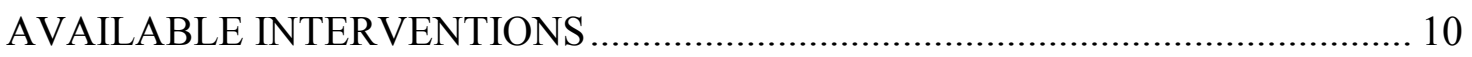

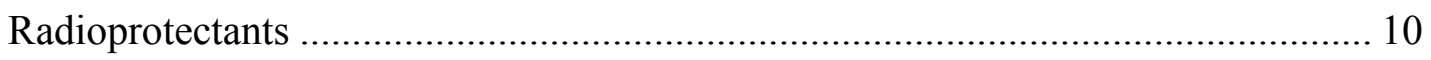

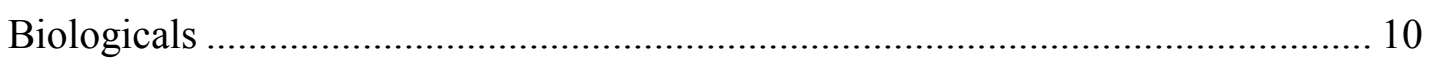

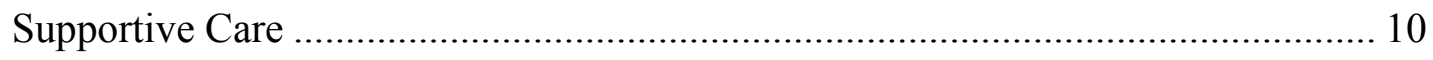

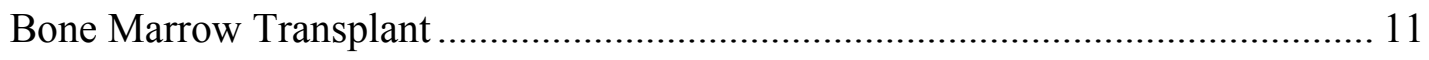

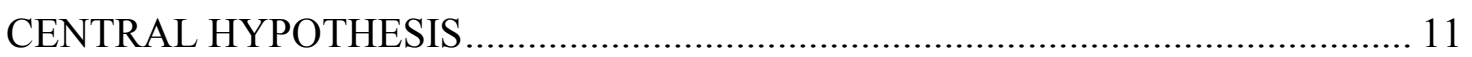

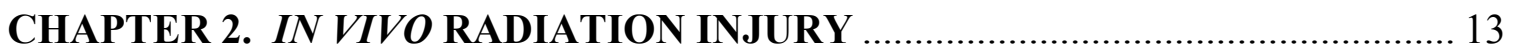

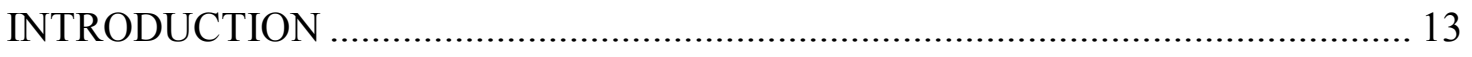

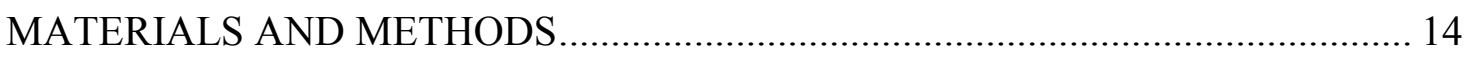

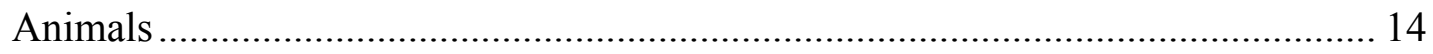

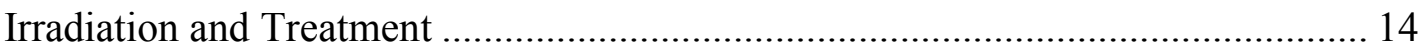

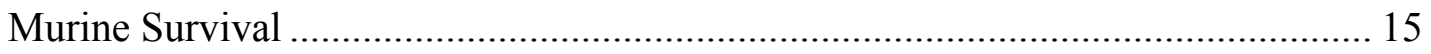

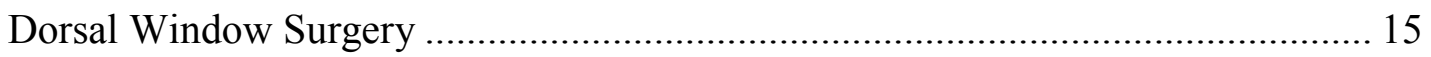

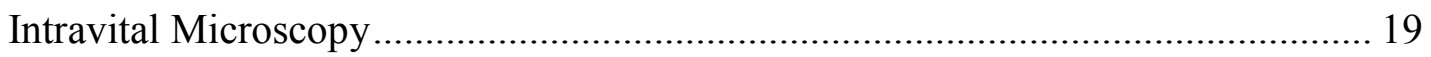

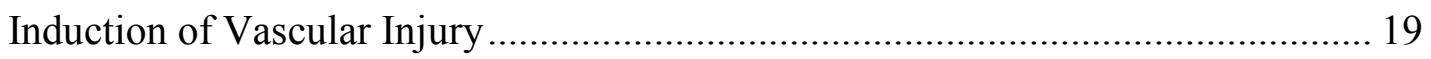




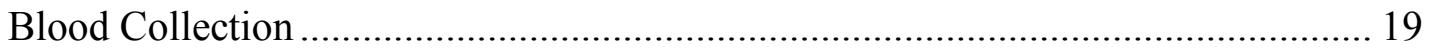

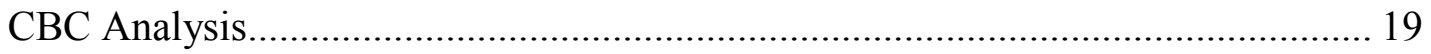

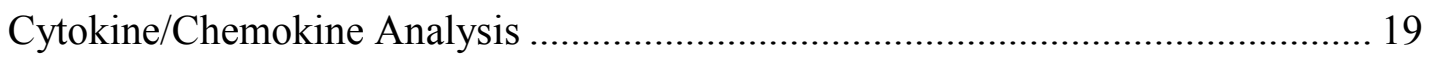

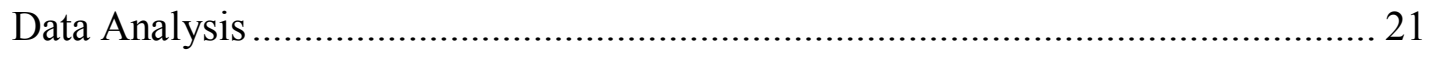

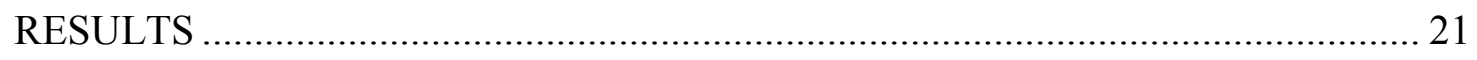

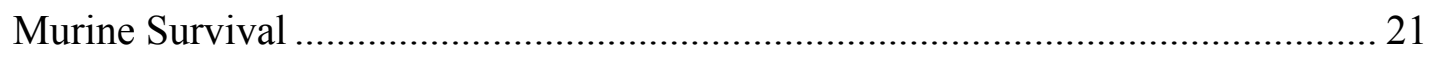

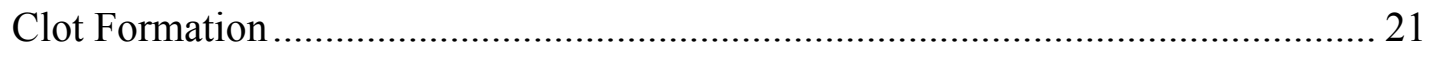

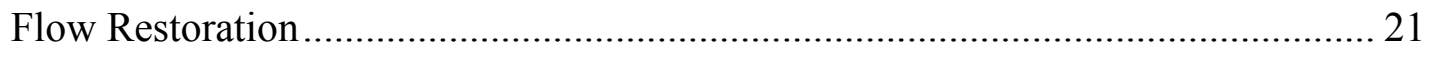

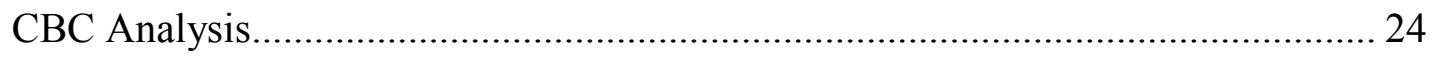

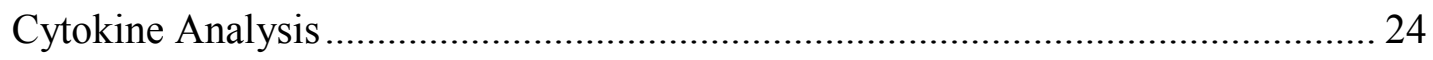

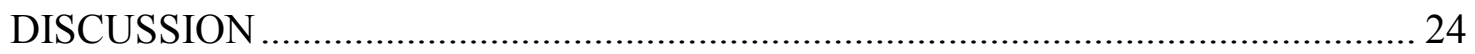

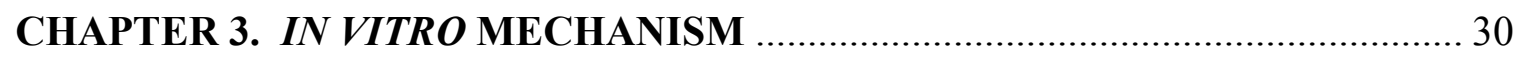

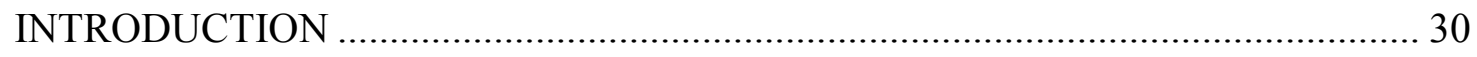

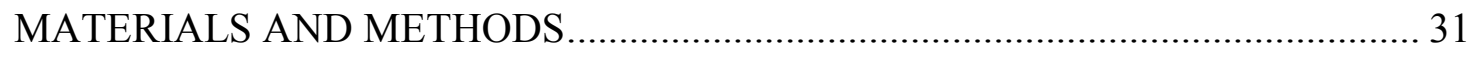

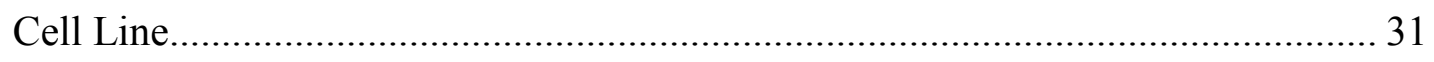

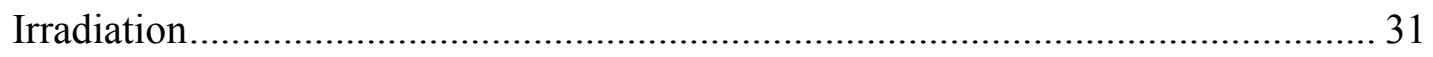

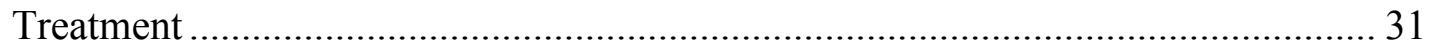

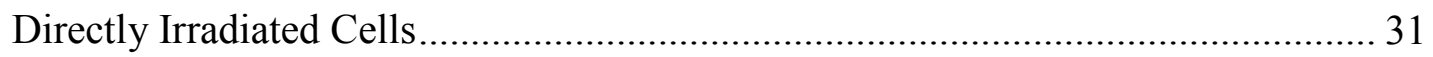

Bystander Effect Cells ................................................................................ 31

Apoptosis ……

DNA Damage

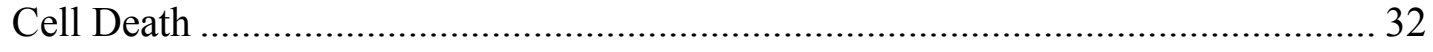

Death Receptor Ligand Quantification ………………….................................. 32

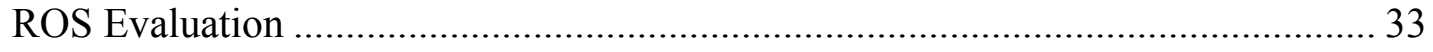

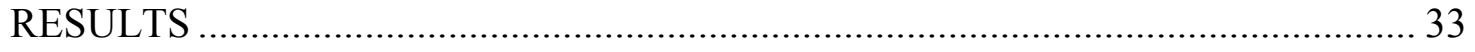

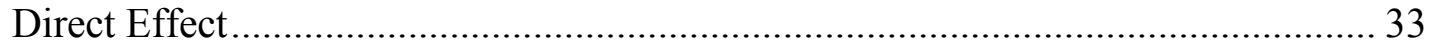

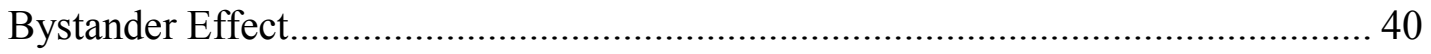

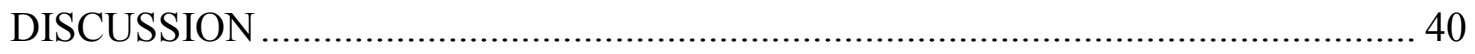

\section{CHAPTER 4. PHARMACOKINETIC MODELING OF NEXT GENERATION}

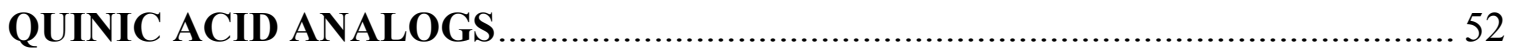

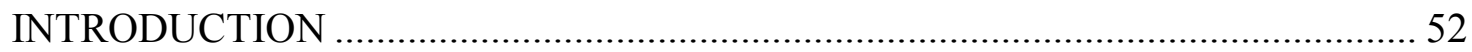

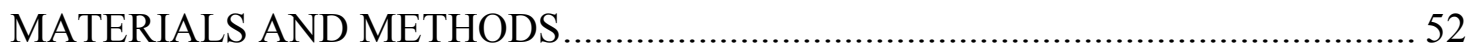

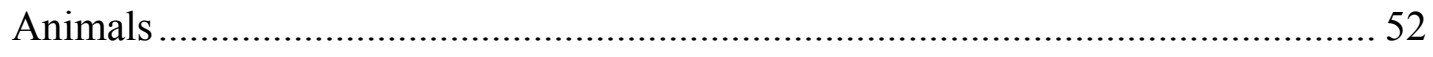




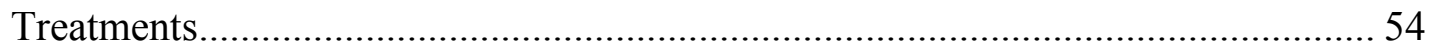

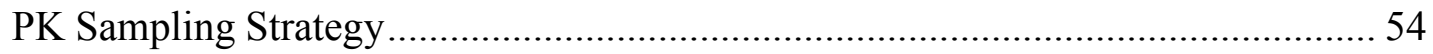

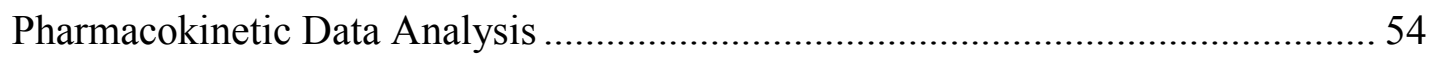

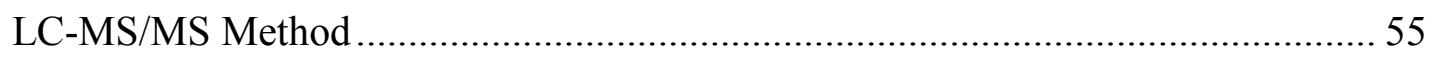

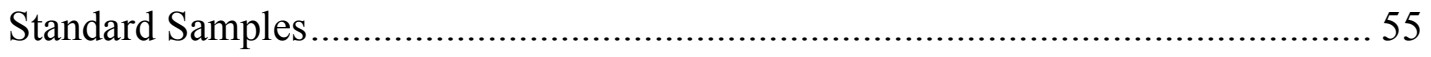

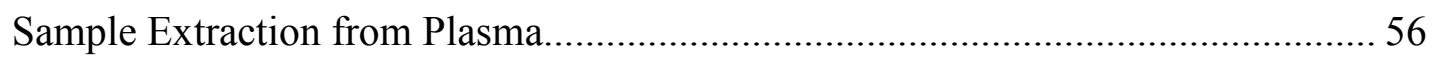

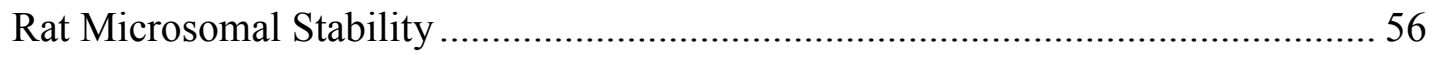

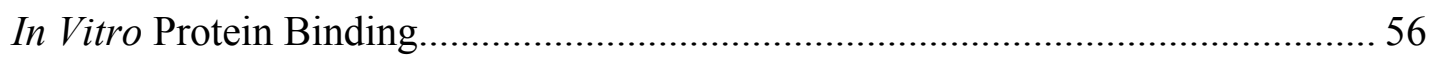

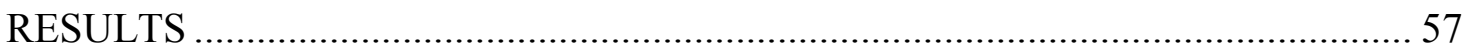

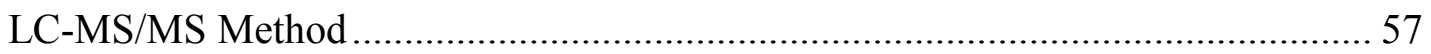

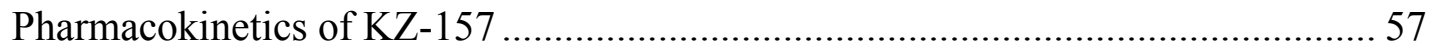

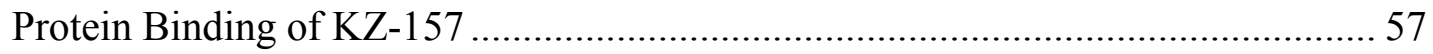

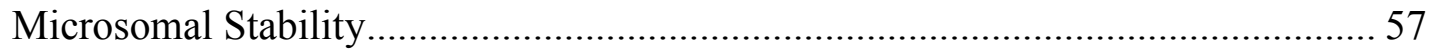

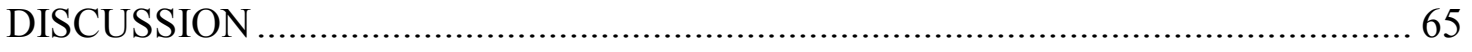

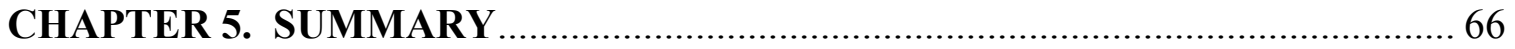

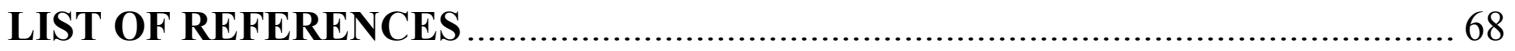

VITA 


\section{LIST OF FIGURES}

Figure 1-1 Radiation Doses Associated with Acute Radiation Syndrome ..................... 2

Figure 1-2 Differientation of Hematopoietic Progenitor Cells.................................... 2

Figure 1-3 Effect of Radiation on Bone Marrow Cellularity ....................................... 3

Figure 1-4 Characteristic Lymphocyte Kinetics after Varying Radiation Doses ........... 6

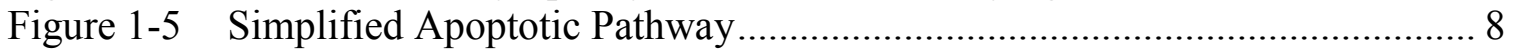

Figure 2-1 Delivering TBI for Survival Study ...................................................... 16

Figure 2-2 Delivering TBI for Imaging Studies .................................................. 17

Figure 2-3 Dorsal Skinfold Window Apparatus...................................................... 18

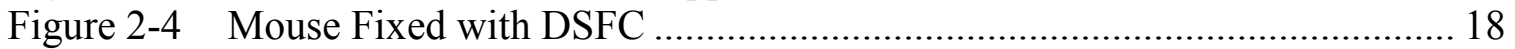

Figure 2-5 Low Magnification Image of Dermal Vasculature ..................................... 20

Figure 2-6 Imaging Study Timeline ........................................................................ 20

Figure 2-7 Effect of KZ-41 on Murine 30 Day Survival............................................ 22

Figure 2-8 Effect of KZ-41 on Radiation-Induced Clotting Abnormalities ................. 23

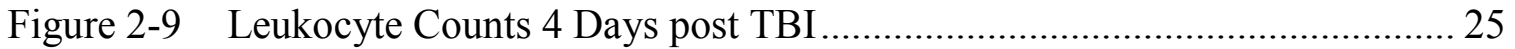

Figure 2-10 Platelet Counts 4 Days post TBI............................................................. 26

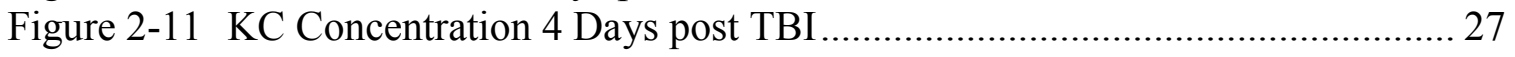

Figure 2-12 TNF- $\alpha$ Concentration 4 Days post TBI ................................................. 28

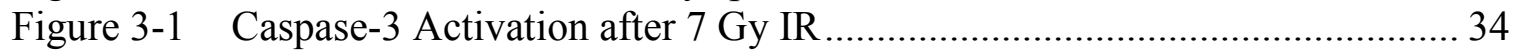

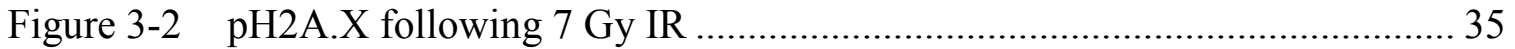

Figure 3-3 Apoptotic Cell Death 24 Hours following 7 Gy IR ................................. 36

Figure 3-4 Effect of KZ-41 at 24 Hours on Radiation-Induced Direct Damage........... 37

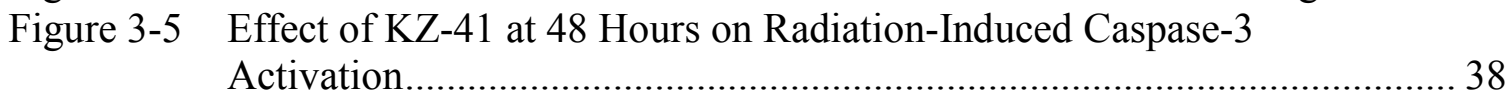

Figure 3-6 Effect of KZ-41 on Apoptotic Cell Death 24 Hours post Radiation

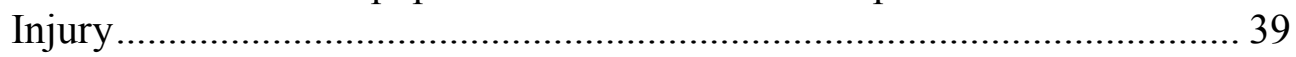

Figure 3-7 TNF- $\alpha$ Concentration following 7 Gy Radiation Exposure........................ 41

Figure 3-8 Effect of KZ-41 on TNF- $\alpha$ Production 48 Hours post Radiation ................ 42

Figure 3-9 Effect of KZ-41 on ROS 24 Hours post Radiation.................................. 43

Figure 3-10 Caspase-3/7 Activity 24 Hours post CM Transfer...................................... 44

Figure 3-11 Stability of Caspase-3/7 in Cell Culture Medium....................................... 45

Figure 3-12 Soluble Caspase-3/7 24 Hours post CM Transfer ..................................... 46

Figure 3-13 Cellular Caspase-3/7 from Bystander Cells 24 Hours post CM Transfer.... 47

Figure 3-14 Effect of KZ-41 on the Radiation-Induced Bystander Effect ..................... 48

Figure 3-15 Chemical Structure of KZ-157 .......................................................... 49

Figure 3-16 Next-Generation Analog Screening ..................................................... 50

Figure 4-1 Chemical Structure of Chlorogenic Acid Derivative KZ-157 .................... 53

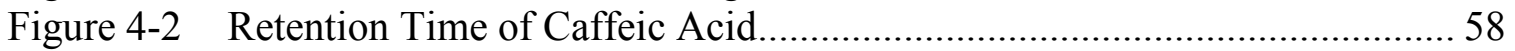

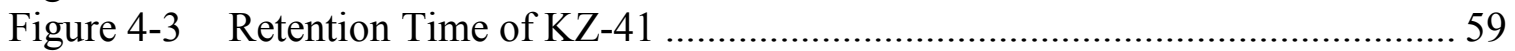

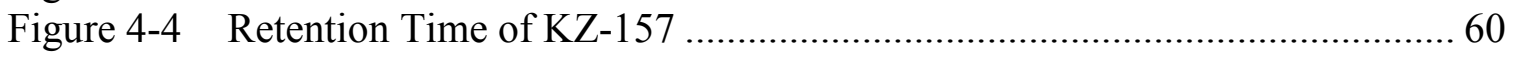

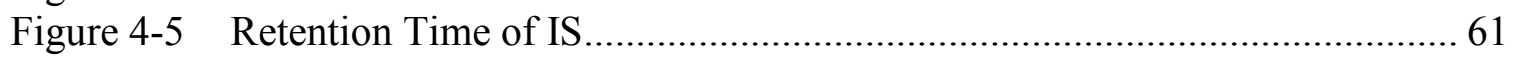

Figure 4-6 Simultaneous Detection of KZ-157 and Primary Metabolites..................... 62 
Figure 4-7 Concentration-Time Profile of KZ-157 after Intravenous

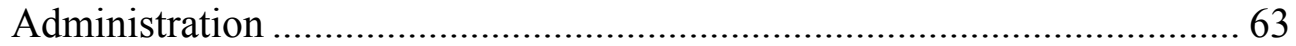

Figure 4-8 Concentration-Time Profile of KZ-157 after Oral Administration ............. 63 


\section{LIST OF ABBREVIATIONS}

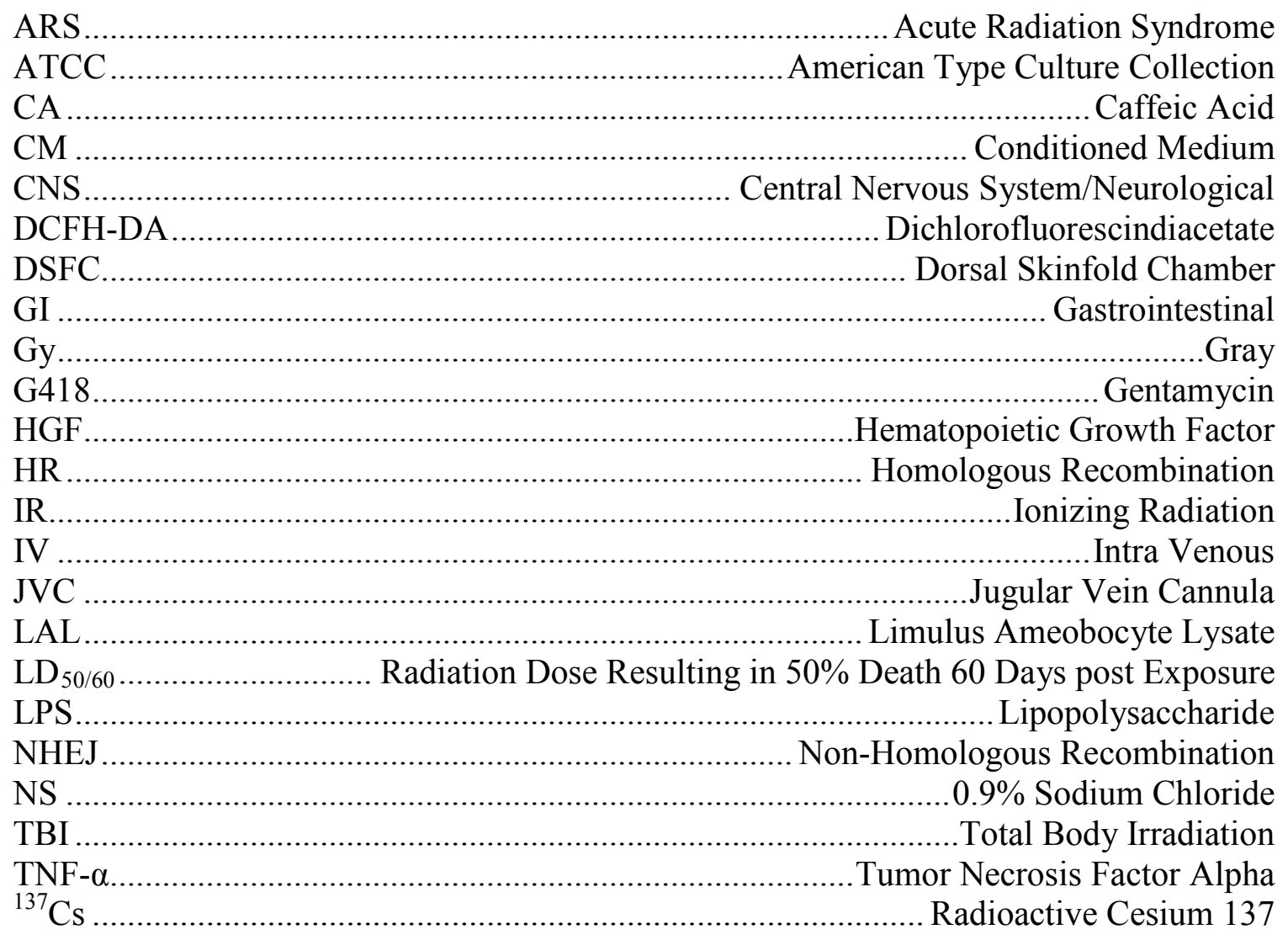




\section{CHAPTER 1. INTRODUCTION}

\section{ACUTE RADIATION SYNDROME}

There is growing concern that the potential for nuclear attack is increasing. Small scale attacks could involve hundreds to thousands of people exposed to total body irradiation (TBI) [1]. Acute radiation syndrome (ARS) results from radiation exposures greater than $1 \mathrm{~Gy}$, to a significant portion of the body delivered at a relatively high dose rate. Such radiation exposure can be from a nuclear accident (e.g., Chernobyl), from a military event (e.g., Hiroshima and Nagasaki), or through a terrorist incident. ARS encompasses overlapping syndromes including hematopoietic (Hem-ARS), gastrointestinal, and neurological syndromes (Figure 1-1). Victims whose TBI dose ranges from 2-6 Gy, known as the hematopoietic syndrome, will be most likely to be saved with treatment, and it is estimated that nearly all persons exposed to doses greater than 6 Gy will die within 30 days of exposure if not treated [2].

\section{Hematopoietic Syndrome}

Hematopoietic stem cells are extremely vulnerable to $\gamma$-irradiation whose damage is the cause of Hem-ARS which occurs at the lowest radiation doses and is observed with increasing severity proportional to radiation dose (Figure 1-2). Symptoms of moderate Hem-ARS is due to exposure to levels of ionizing radiation greater than 1-2 Gray, and manifests as bone marrow damage (Figure 1-3) [3]. Severe Hem-ARS is seen at ionizing radiation doses from 4 Gy $[2,4]$. It is estimated that without supportive care (antibiotics and transfusions), a radiation dose of approximately 3.5-4.5 Gy will result in death of $50 \%$ of persons exposed within 60 days $\left(\operatorname{LD}_{50 / 60}\right)[3,5,6]$. While ARS can affect many organ systems (e.g., dermal, cardiovascular) primary causes of morbidity and mortality in Hem-ARS are related to pancytopenia. Death resulting from sepsis results from a decrease in innate immunity, both the numbers of circulating leukocytes and possibly the function $[7,8]$. Hemorrhage associated mortality can be correlated with a significant decrease in circulating platelet levels and magakaryocyte precursors. In the event of a nuclear accident or attack, there will be other injuries in addition to the radiation exposure. It has been estimated that as many as $60-70 \%$ of ARS patients will have other injuries, and these patients will have a significantly lower $\mathrm{LD}_{50 / 60}$ [5]. Additional injuries increase the probability of introduction of infection that a compromised hematopoietic system cannot combat. Hematopoietic recovery must be attained or the patient will have no chance to recover from other injuries [9]. Therefore, hematopoietic support is essential at all radiation doses relevant to induce ARS.

\section{Gastrointestinal Syndrome}

Total body doses above approximately 8 Gy will involve serious injury to the gastrointestinal (GI) system, contributing to mortality [10]. Radiation doses from around 


\begin{tabular}{|cccc||}
\hline $\begin{array}{c}\text { Hematopoietic } \\
\text { (HEM-ARS) }\end{array}$ & $\downarrow$ & $\begin{array}{c}\text { Gastrointestinal } \\
\text { (GI-ARS) }\end{array}$ & $\downarrow$ \\
\hline $\begin{array}{c}\text { Radiation Dose } \\
\text { (Human, Gy) }\end{array}$ & 6 & 10 & CNS \\
\hline
\end{tabular}

Figure 1-1 Radiation Doses Associated with Acute Radiation Syndrome.

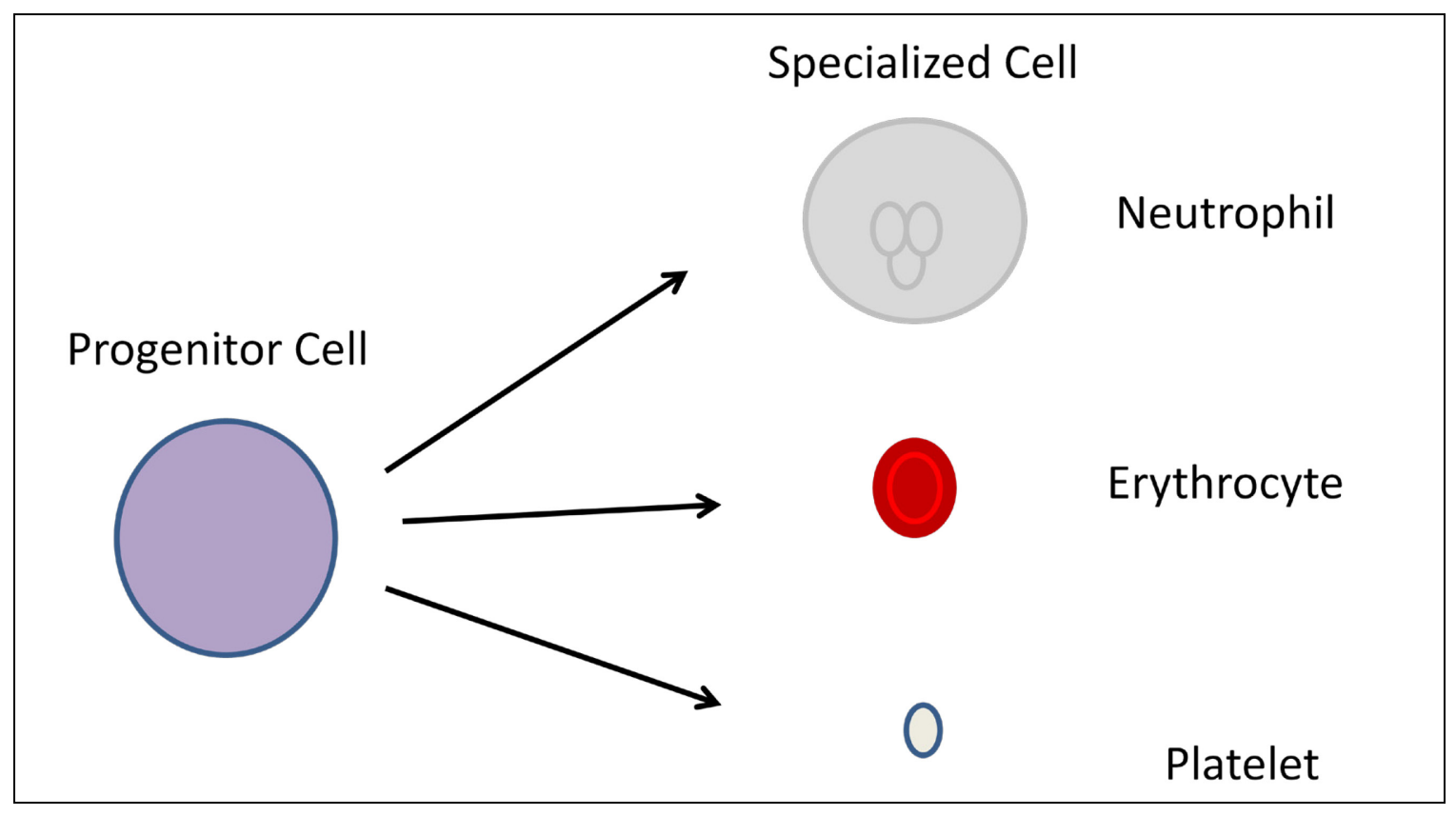

Figure 1-2 Differientation of Hematopoietic Progenitor Cells. 


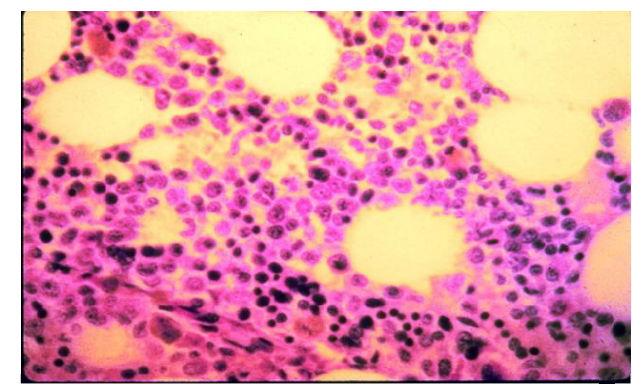

Normal

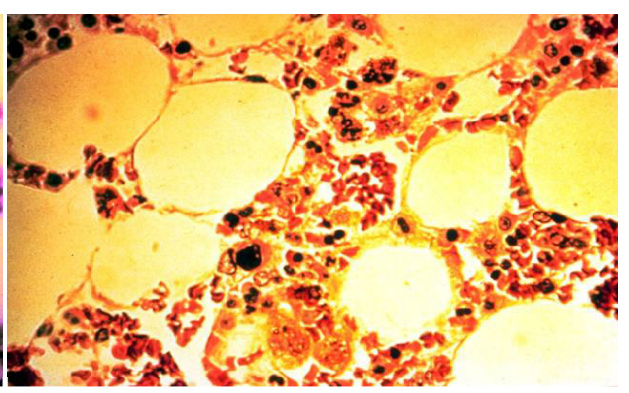

Reduced After IR

Figure 1-3 Effect of Radiation on Bone Marrow Cellularity.

Normal bone marrow cellularity is dense as demonstrated by the panel on the left. The panel on the right indicates reduced cellularity in the bone marrow after high dose irradiation. 
8-30 Gy elicit effects known as the GI syndrome. Damage to intestinal mucosal stem cells is the hallmark of the GI syndrome. The result of the damage to the intestine is decreased barrier function and abnormal absorption. Clinical symptoms of GI-ARS include nausea, vomiting, and diarrhea in addition to those of the hematopoietic syndrome, impaired immune function being of particular importance [2]. Since ARS sub-syndromes overlap, patients faced with the GI syndrome are still highly susceptible to hemorrhage and infections due to a depressed immune system. Decreased intestinal epithelial barrier function results in an increased risk of bacterial translocation into the blood, and coupled with a diminished ability to combat infection, results in an increased incidence of sepsis and death and a marked shift in the mortality curve.

\section{Neurological Syndrome}

Total body doses above approximately 30 Gy will elicit the neurological (CNS) syndrome due primarily to hypotension and cerebral edema. At CNS syndrome doses, cardiovascular damage also occurs and death occurs within hours to days [2]. Symptoms include loss of consciousness in addition to all of those experienced in the hematopoietic and gastrointestinal syndrome with a more severe and rapid onset. The CNS syndrome is the least studied due to the fact that very few accident victims worldwide have encountered this extremely high dose. It must be noted, however, that even at nonlethal ARS radiation doses neurological effects may be seen after several days manifested by lower response rates $[11,12]$. Under the current understanding of the biophysical pathways and effects elicited by these very high doses of TBI, it is unlikely that a survival advantage could be obtained.

\section{Clinical Progression of ARS}

Time to Onset. Symptoms of ARS may be seen as early as 1-2 hours after exposure. Symptoms include vomiting, diarrhea, headache, altered consciousness, fever, and hematological changes. As radiation dose increases, symptoms become more severe and appear earlier $[2,8]$. There are three phases describing the progression of ARS: (1) Prodromal, (2) Latent phase, and (3) Critical Phase. The prodromal phase occurs very soon after exposure and may include mild to moderate physical symptoms, but is rarely lethal. Following the prodromal phase, there is a latent period lasting a variable amount of time, depending on the absorbed dose. The length of the latent period ranges from 0 35 days and is inversely related to the radiation dose. The latent phase is often free from manifested symptoms. The critical phase is defined as the peak of bone marrow depression resulting in leucopenia and thrombocytopenia and occurs sooner as the dose increases. Death due to infection and hemorrhage tend to correlate with this nadir $[2,13]$.

Hematological Kinetics. The myelosupression resulting from TBI is dosedependent [13]. Irreversible injury shows initial granulocytosis and progressive decline of cell counts to nadir around day 6. The initial granulocytosis is due to rapid mobilization of granulocytes from reserve pools, and the approximate 4 day maturation 
time of granulocytes in the bone marrow. Assuming irreversible injury (all pre-cursor cells in the bone marrow have been destroyed), once these cells have appeared in the blood, the decline occurs rapidly. A normal lymphocyte count in an adult is 1.4-3.9 $\mathrm{x}$ $10^{4} / \mathrm{L}$ [14]. Lymphocytes show progressive decline and nadir within 48 hours. This rapid decline is not a result of radiation sensitivity of the lymphocyte, but rather an impaired ability to re-circulate. Therefore, lymphocyte decline is not necessarily indicative of degree of bone marrow damage, however, a 50\% decline at 24 hours after exposure may indicate a lethal radiation dose has been absorbed (Figure 1-4) [14]. Platelets show progressive decline over the first 10 days with nadir at day 10 . This linear decline in platelets over 10 days is reflective of the survival time of circulating platelets and indicative of all megakaryocyte precursors in the bone marrow having been destroyed. Reversible injury shows moderate initial granulocytosis and decline between days 4-10, an abortive recovery and nadir at days 20-30. Lymphocytes show nadir within 2 days, and slow recovery (over $\sim 25$ days). Platelets show an initial 10 day shoulder, and then decline and nadir near day 20 that lasts through day $30[3,8,9]$.

Manifestations. Symptoms of moderate Hem-ARS is due to exposure to levels of ionizing radiation around 3-4 Gray. Severe Hem-ARS is seen at ionizing radiation doses from 4 Gy $[2,4]$. While ARS can affect many organ systems (e.g., dermal, cardiovascular), acute lethality generally results from destruction of the hematopoietic system ("Hem-ARS") or related complications (e.g. sepsis).

\section{RADIATION INJURY}

Ionizing radiation (IR) may consist of particulate or electromagnetic radiation. Simulation of ARS for drug development and research purposes likely involves electromagnetic radiation. IR comes from the portion of the electromagnetic spectrum characterized by high frequency and short wavelength. Biological damage results from the absorption and deposition of energy in cells and tissues. This energy deposition occurs non-homogenously and therefore requires much less total energy to cause damage than non-ionizing radiation [15]. Damage induced by ionizing radiation encompasses two primary categories: initial damage and bystander damage. The importance of understanding the effect a novel radiomitigant has on mitigating the bystander effect may help to expand the treatment window that the compound may be first administered and retain efficacy.

\section{Initial Effect}

The two causes of initial damage from directly irradiating cells are the charged particles that directly interact with cellular structures (direct action), and free radical generation which induces double strand breaks and oxidative stress (indirect action). The energy deposited in a cell when it is irradiated is primarily absorbed by water leading to the nearly instantaneous production of radical intermediates. These intermediates are then free to react with other cellular structures. 


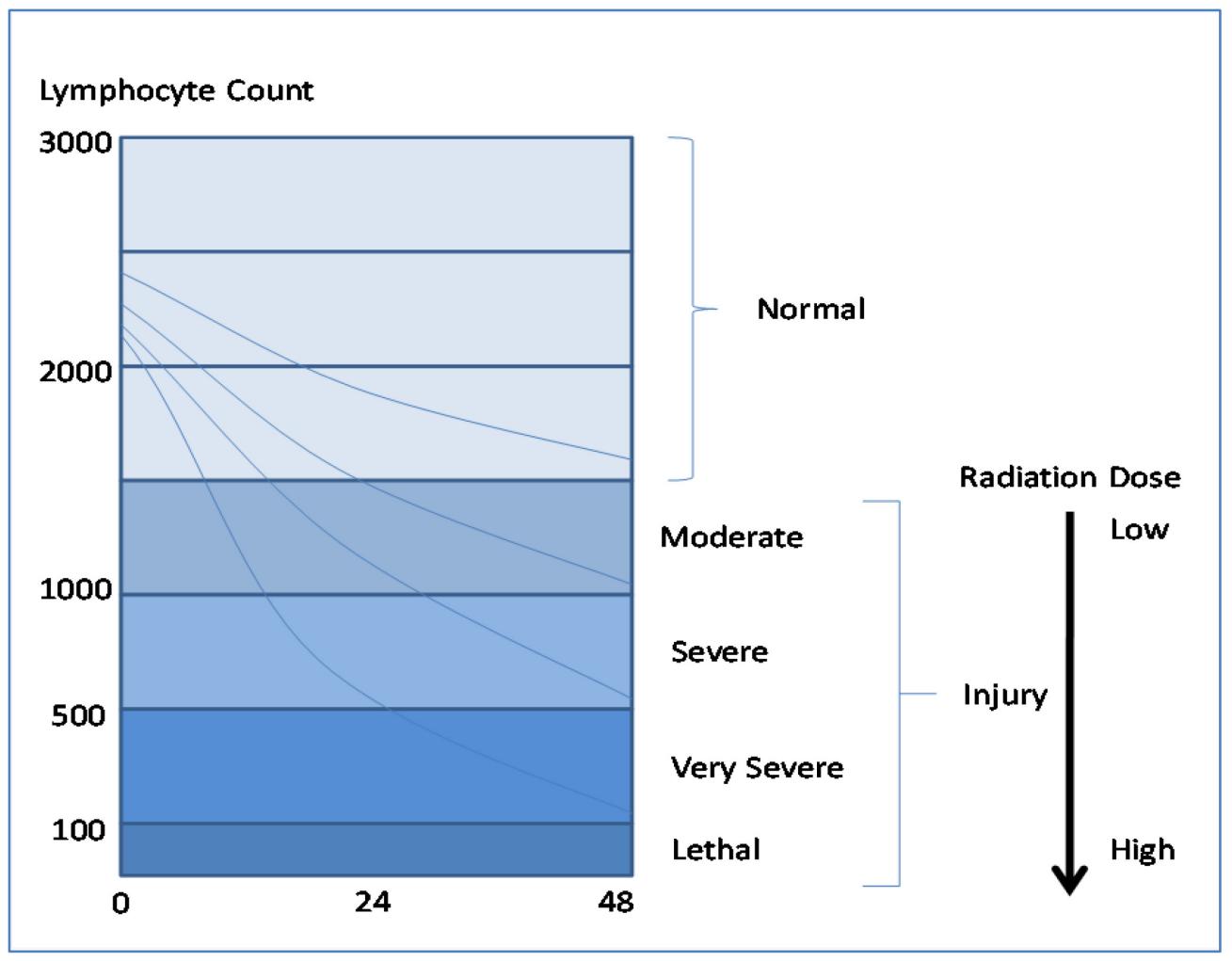

Figure 1-4 Characteristic Lymphocyte Kinetics after Varying Radiation Doses. 


\section{Bystander Effect}

Signals are produced by the initially damaged cells and are transferred through media and to a lesser extent through gap junctions. The damage caused to unirradiated cells through these signals is a phenomenon known as bystander or non-targeted effects. Generally, the term 'Bystander Effect' is used in the setting of non-therapeutic use of radiation such as in an accident or nuclear attack because there is no intended 'target' for the radiation.

\section{Mechanisms of Radiation-Induced Cell Death}

Apoptosis. The process of programmed cell death known as apoptosis plays a crucial role in the biological effects caused by ARS. It is involved in both normal cellular regulations in organs and in disease states. Organs which demonstrate high proliferative and hematopoietic activity have a higher baseline level of apoptosis.

Apoptosis is characterized by specific cellular changes including chromatin condensation, mitochondrial swelling, DNA cleavage, and loss of membrane integrity. Apoptosis may proceed via a Caspase- 8 or Caspase- 9 dependent pathway; however they converge during execution initiated by cleavage of Caspase-3 (Figure 1-5) [16].

Intrinsic. Apoptotic cell death which is initiated from within the cell results in death via the intrinsic pathway, also known as mitochondrial or Caspase-9 dependent cell death. It has recently been reported that death from Hem-ARS requires intrinsic apoptosis [17].

Extrinsic. Apoptotic cell death which is initiated outside the cell results in death via the extrinsic pathway, also known as Caspase- 8 dependent cell death or death receptor pathway. Extrinsic apoptosis occurs without mitochondrial involvement.

Mitotic Cell Death. The process known as mitotic cell death or mitotic catastrophe results when a mitotic arrest occurs during which intrinsic apoptotic pathway hallmarks become activated.

\section{COMBINED INJURY}

In the aftermath of a nuclear detonation it is feasible that there would be combination injuries involving damage from both the radiation coupled with wounds of varying severity. TBI in combination with vascular, dermal, or muscular injuries will decrease survival. Due to the importance of considering realistic scenarios, a compound being considered for an ARS indication must contemplate efficacy in a combined injury setting. 


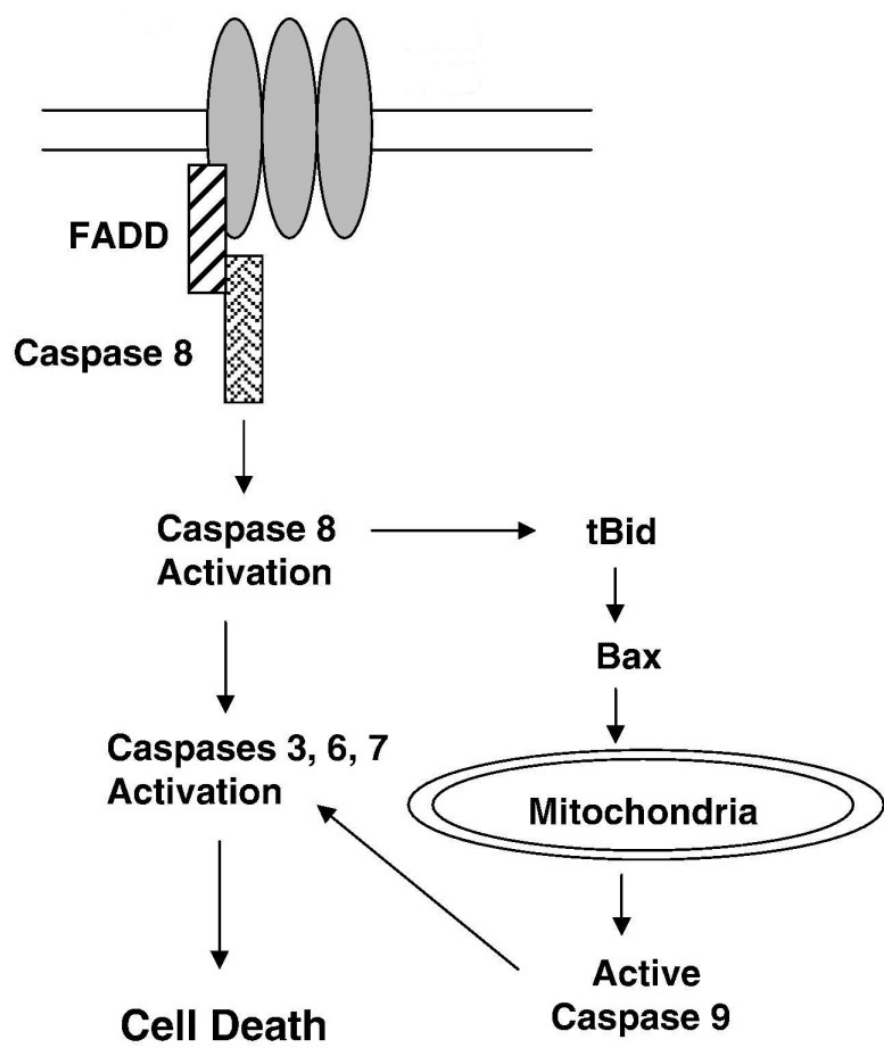

Figure 1-5 Simplified Apoptotic Pathway. 


\section{BIOMARKERS}

The use of biomarkers in the setting of ARS has been primarily investigated for use in dosimetry $[18,19]$. Renewed interest in biomarkers for use as pharmacodynamic or surrogate endpoint markers emerges in light of the quest for development of pharmaceutical treatments for ARS.

\section{Circulating Blood Counts}

The most widely used biomarker is complete blood counts (CBC). $\mathrm{CBC}$ analysis has been used both to indicate dosimetry and therapeutic response [13, 20-22]. Specifically, the kinetics of circulating blood cells (granulocytes, lymphocytes, and platelets) are used to retrospectively estimate radiation dose, extent of hematopoietic injury, and likelihood of spontaneous recovery without treatment $[9,23]$. CBC analysis is very useful, in indicating the extent of damage, however, due to reports that function may be impaired despite increased circulating numbers, $\mathrm{CBC}$ counts may not be the best way to evaluate recovery $[7,22]$.

\section{Amylase}

Serum amylase is used as an indicator of damage to the parotid gland by radiation $[24,25]$. Serum amylase is the result of interphase cell death of serous salivary cells. It must be measured within 0.5-2 days of the initial radiation event in order to correlate elevated levels with exposure $[14,24]$. In non-human primates mates, significant intersubject variability was observed when measuring serum amylase, with somewhat less variability in plasma amylase [14]. Serum amylase returns to baseline within a few days after radiation exposure, thereby limiting the use of amylase to predict efficacy of countermeasures.

\section{Flt3-Ligand}

The Flt3-ligand (FL) is a $34 \mathrm{kDa}$ cytokine which works synergistically with other hematopoietic growth factors (HGF) to induce the proliferation of pluripotent hematopoietic progenitor and stem cells $[19,26]$. The Flt3-ligand has been used in a clinical setting as late as day 20 post TBI exposure as a measure of the extent of hematopoietic exposure and correlates with hematopoietic nadir [27].

\section{Citrulline}

Citrulline is an amino acid produced by enterocytes and used as an indicator of gastrointestinal damage [27]. It may be measured in plasma by chromatographic 
methods, and has been shown to reflect actual mucosal damage rather than absolute radiation dose.

\section{AVAILABLE INTERVENTIONS}

Although there is a single product, amifostine, approved by the FDA for administration prior to radiation, there are currently no known approved drugs that can be administered after TBI exposure to enhance survival due to ARS.

\section{Radioprotectants}

Prophylactic potassium iodide administration to protect the thyroid from exposure to excess ${ }^{132}$ I, Chelating agents such as diethylene triamine pentaacetic acid ("DTPA") to decrease exposure by binding and promoting urinary excretion and similarly, laxatives, and absorbing agents such as Prussian blue to limit GI absorption or high oral doses of calcium to compete with intestinal absorption of milk contaminated with strontium 90. Amifostine is a thiol compound under investigation as a radioprotectant. It has been used to prevent mucositis caused by head-and-neck irradiation but must be administered in advance of radiation exposure. Furthermore, its side effects (hypotension) would limit its use in emergency first responders [28].

\section{Biologicals}

Growth factors are present abundantly in the circulation of people suffering from radiation injury [9]. The use of biological products such as G-CSF for the treatment of ARS is controversial. Agents that have not been approved by the FDA as radiomitigants but are under investigation as regenerators include cytokines such as granulocyte colony stimulating factor ("G-CSF") and granulocyte-macrophage colony stimulating factor ("GM-CSF"). Both must be given parenterally in a sophisticated medical setting as with bone marrow transplant. Various lipopeptide agonists of Toll-like Receptors (e.g., TRL2/TRL6) are under investigation as an aid to bone marrow transplantation. Other TRL agonists are under investigation for treatment of Hem-ARS and oral mucositis following head and neck therapeutic irradiation.

\section{Supportive Care}

The use of supportive care is critical for optimum survival outcome in ARS, particularly at low TBI doses. Supportive care includes administration of antibiotics, fluids, blood products, isolation from secondary infections, and regulation of body temperature. 


\section{Bone Marrow Transplant}

With regard to Hem-ARS, bone-marrow transplants (BMT) have been used for irradiated victims without success [1-3]. 27 of the 31 patients that received BMT died. The four survivors rejected the transplant and spontaneously repopulated with their own stem cells $[1,4]$. Furthermore, bone marrow transplants would not be practical after a large scale disaster involving large numbers of patients requiring treatment.

\section{CENTRAL HYPOTHESIS}

The ideal treatment for ARS would be a physically stable compound that could be easily and rapidly administered to large numbers of people exposed to a range of radiation doses. The treatment should retain effectiveness when administered as a single dose no sooner than 24 hours after exposure. The treatment should result primarily in improvement in survival and secondarily in alleviation or improvement in associated morbidities.

A drug approved for use in the treatment of ARS must attain approval through the path set forth by the so-called "Animal Rule" because ethical considerations prevent preapproval efficacy testing in human subjects. The "Animal Rule" is encompasses four primary pillars: (1) understanding the mechanism of the toxicity of the substance (radiation) and its prevention or substantial reduction by the product (2) demonstrating the effect in more than one animal species that is expected to react with a response that is predictive for humans (3) demonstrating efficacy in the animal study endpoint that is clearly related to the desired benefits in humans, and (4) characterizing the pharmacokinetics and pharmacodynamics in such a way that allows selection of an effective human dose.

Specific aim 1 (Chapter 2) focused on identifying primary endpoints in established and novel in vivo radiation models. In this aim we tested our lead compound, KZ-41, for efficacy on our primary endpoint- 30 day mortality. We also developed an in vivo model to evaluate the effect of radiation on vascular injury, specifically clotting abnormalities. We tested the efficacy of KZ-41 in restoring thrombus formation after radiation in this novel model.

In specific aim 2 (Chapter 3), we wanted to focus on the pathways involved in indirect damage caused by radiation. Our aim was to develop a model that would allow testing of agents administered at least 24 hours after the initial radiation injury. To this end, we developed an in vitro bystander effect model using human U937 cells, and tested the effect KZ-41 had on pathways involved in apoptotic cell death. We also compared the effect next-generation radiomitigating compounds to KZ-41 in the bystander effect model.

Specific aim 3 (Chapter 4) focused on initial pharmaceutical profiling of nextgeneration radiomitigant KZ-157. We first developed a LC-MS/MS assay to measure 
KZ-157 and its primary metabolites in biological matrices. We then measured protein binding, susceptibility to P450 metabolism, and characterized the concentration-time profile after i.v. and oral administration to rats. 


\section{CHAPTER 2. IN VIVO RADIATION INJURY}

\section{INTRODUCTION}

There is growing concern that the potential for nuclear attack is increasing. Small scale attacks could involve hundreds to thousands of people exposed to total body irradiation (TBI) [1]. Radiation exposures greater than $1 \mathrm{~Gy}$, involving a significant portion of the body and delivered at a relatively high dose rate induces Acute Radiation Syndrome (ARS). Such radiation exposure can be from a nuclear accident (e.g., Chernobyl), from a military event (e.g., Hiroshima and Nagasaki), or through a terrorist incident. Victims whose TBI dose ranges from 2-6 Gy, eliciting the response known as the hematopoietic syndrome, will be most likely to be saved with treatment. It is estimated that without supportive care (antibiotics and transfusions), a radiation dose of approximately 3.5-4.5 Gy will result in death of $50 \%$ of persons exposed within 60 days $[3,5,6]$, and that nearly all untreated persons exposed to doses greater than $6 \mathrm{~Gy}$ will die within 30 days of exposure [2]. The primary causes of death of persons suffering from Hem-ARS are hemorrhage and infection due to the dose-dependent damage to the hematopoietic stem cells.

There is an urgent unmet therapeutic need for radiomitigating (administered at least 24 hours after radiation injury) and radioprotecting compounds (administered less than 24 hours after radiation injury) which produce an improvement in survival.

Although the endpoint of improving survival seems to be clear-cut, in situations such as nuclear accident or attack, there will likely be additional injuries secondary to the radiation exposure. It has been estimated that as many as $60-70 \%$ of ARS patients will have other injuries, and these patients will have a significantly lower $\mathrm{LD}_{50 / 60}$, as secondary injury negatively impacted survival $[5,29]$. Additional injuries increase the probability of introduction of infection that a compromised hematopoietic system cannot combat. Hematopoietic recovery must be attained or the patient will have no chance to recover from other injuries [9]. Therefore, hematopoietic support is essential at all radiation doses relevant to induce ARS.

Little is known about the effects of vascular injury in the setting of high doses of total body irradiation simulating a nuclear accident or attack. Total body irradiation delivered in large doses, to most or all of the body in a short period of time induces ARS and causes widespread effects in the exposed organism. The hematopoietic syndrome occurs even at the lowest doses that cause ARS, at approximately an $\mathrm{LD}_{50 / 30}$ in mammals [30]. The hematopoietic syndrome encompasses two sub-syndromes characterized by leucopenia and thrombocytopenia. Our focus in the combined radiation model is to evaluate the initial steps of wound healing in the vasculature primarily early thrombus formation, translating to subjects presenting with the hematopoietic syndrome who die from hemorrhage caused by thrombocytopenia [30]. 
Radiation injury models are often established where they are routinely implemented due to additional variables that must be characterized including type of electromagnetic radiation ( $\gamma$-ray vs. $\mathrm{x}$-ray), source $\left({ }^{60} \mathrm{Co}\right.$ vs. ${ }^{137} \mathrm{Cs}$ ), dose rate, and normal bacterial flora found in the local vivarium. It is nearly impossible in a non-GLP facility to directly implement an established model without minor modifications. Therefore, it is of critical importance to clearly outline and account for as many variables as possible when designing a radiation model.

Unique challenges are presented when developing and characterizing a combined injury model in addition to those discussed for radiation injury models. The desire to produce the most robust model must be delicately weighed against approaching relevant radiation doses and endpoints. Also, it is widely accepted that excessive handling and manipulations cause a shift in both the radiation dose required to cause lethality and the time to reach such an endpoint. This is of particular importance when dealing with smaller model types, such as mice. Special consideration must be taken when choosing the timing of both insults, radiation dose used, supportive care required, extent of secondary insult, and timing (in relation to injuries) of the endpoint measurement. All of these factors are intimately connected, and changes in one parameter must not be made after the model is established without measuring the impact on all other relevant factors.

\section{MATERIALS AND METHODS}

\section{Animals}

Animals were purchased from Jackson Labs (Bar Harbor, ME). Animals were maintained under specific pathogen-free conditions with controlled temperature $\left(22^{\circ} \mathrm{C}\right)$ and humidity (50\%) and on 12-hr light/dark cycles. Animals were allowed a minimum acclimation period of 72 hours from time of arrival prior to subjection to any procedure. Standard laboratory food and tap water were available ad libitum.

Survival Study. Female C57/BL/6 mice at least 12 weeks old were used. These studies were reviewed and approved by the Animal Care and Use Committee of the Veterans Hospital, Memphis, TN.

Imaging Study. Male C57/BL/6 mice weighing 25-30g were used. These studies were reviewed and approved by the Animal Care and Use Committee of the University of Tennessee Health Science Center.

\section{Irradiation and Treatment}

A radioactive cesium $137\left({ }^{137} \mathrm{Cs}\right)$ source was used for irradiation. $\mathrm{KZ}-41$ was prepared as a solution in $0.9 \%$ Sodium Chloride at a concentration of $10 \mathrm{mg}$ per $\mathrm{mL}$. KZ-41 was synthesized as previously described in Dr. Duane Miller's laboratory [5]. 
Survival Study. Animals were irradiated at a dose rate of approximately 3 Gy/minute. A dose of 6.5 Gy was delivered uniformly to the entire body of each animal. Animals were irradiated un-anesthetized in groups of 5 on a rotating platform (Figure 2-1). Treatments were administered as a single dose 25 hours after TBI. The "treatment" group received KZ-41 (100 mg/kg, s.q.), the "vehicle" group received $0.9 \%$ Sodium Chloride (10 $\mu \mathrm{L}$ per 1 gram body weight, s.q.).

Imaging Study. Animals were irradiated at a dose rate of approximately 8 Gy/minute. A dose of 6 Gy was delivered uniformly to the entire body of each animal. Animals were irradiated individually in the upright position on a rotating platform while anesthetized (Figure 2-2). Animals were divided into 3 groups for treatment. Treatment began 12 hours after TBI, and continued every 24 hours for the duration of the study. The "treatment" group received KZ-41 (100 mg/kg, i.p.), the "vehicle" group received $0.9 \%$ Sodium Chloride ( $10 \mu \mathrm{L}$ per 1 gram body weight), and the "sham" group received no injection.

\section{Murine Survival}

After irradiation, and administration of treatement 26 hours after TBI, animals were monitored closely. The number of animals surviving in each group was documented for a period of 30 days.

\section{Dorsal Window Surgery}

Surgery was carried out as described previously [3, 4] with modifications. All surgical procedures were carried out aseptically. After the animal was anesthetized, a suitable area of skin on the dorsum was marked for hair removal with a clipper followed by use of hair remover lotion. The surgical area was disinfected with alcohol and betadine. A thin dorsal skin fold was lifted from the back and sandwiched between two titanium plates (Figure 2-3). The titanium plates were firmly fixed with three screws and nuts through the holes made in the fold. The skin was sutured to the top of the plates for added stability. A circular section of the skin on one side of the skin fold along with its cutaneous tissue and fascia was incised through the window of the dorsal skinfold window chamber (DSFC) assembly to expose the blood vessels and the striated muscle of the opposing skin of the dorsal skin fold. Antibiotic ointment containing bacitracin zinc, polymyxin B sulfate and neomycin sulfate was applied to the edges of the incised wound and a glass window fixed with a snap ring on top of the exposed area inside the DSFC

(Figure 2-4). Animals received analgesic every 12 hours for the first 48 hours after surgery. Animals were allowed 3 days to recover after dorsal skin fold chamber fixation prior to baseline measurements and radiation. 


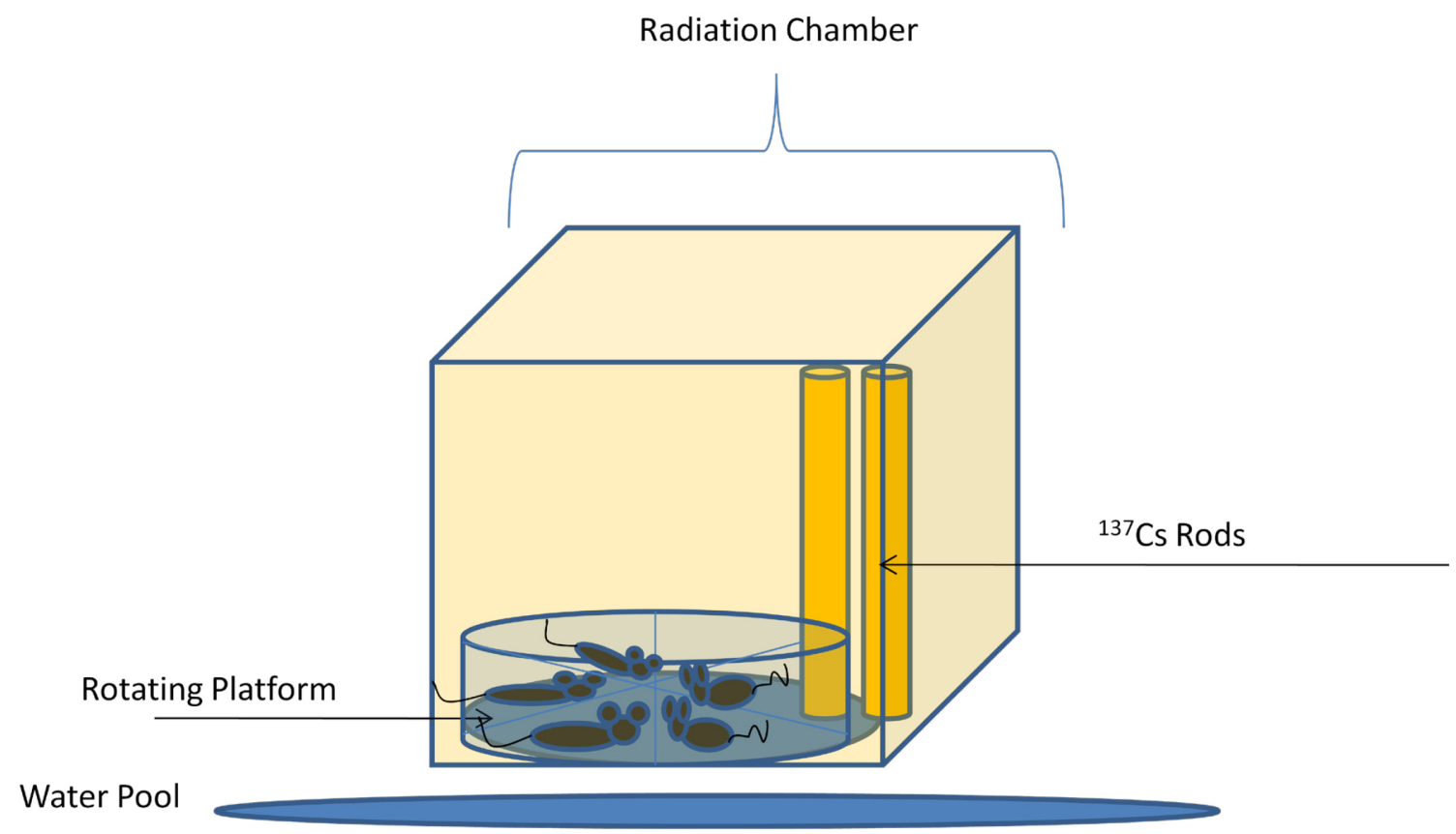

Figure 2-1 Delivering TBI for Survival Study.

Unshielded mice were irradiated unanesthetized 5 at a time on a rotating platform. The dose rate was approximately $3 \mathrm{~Gy} / \mathrm{min}$ and a total dose of $6.5 \mathrm{~Gy}$ (LD80/30) was delivered. 


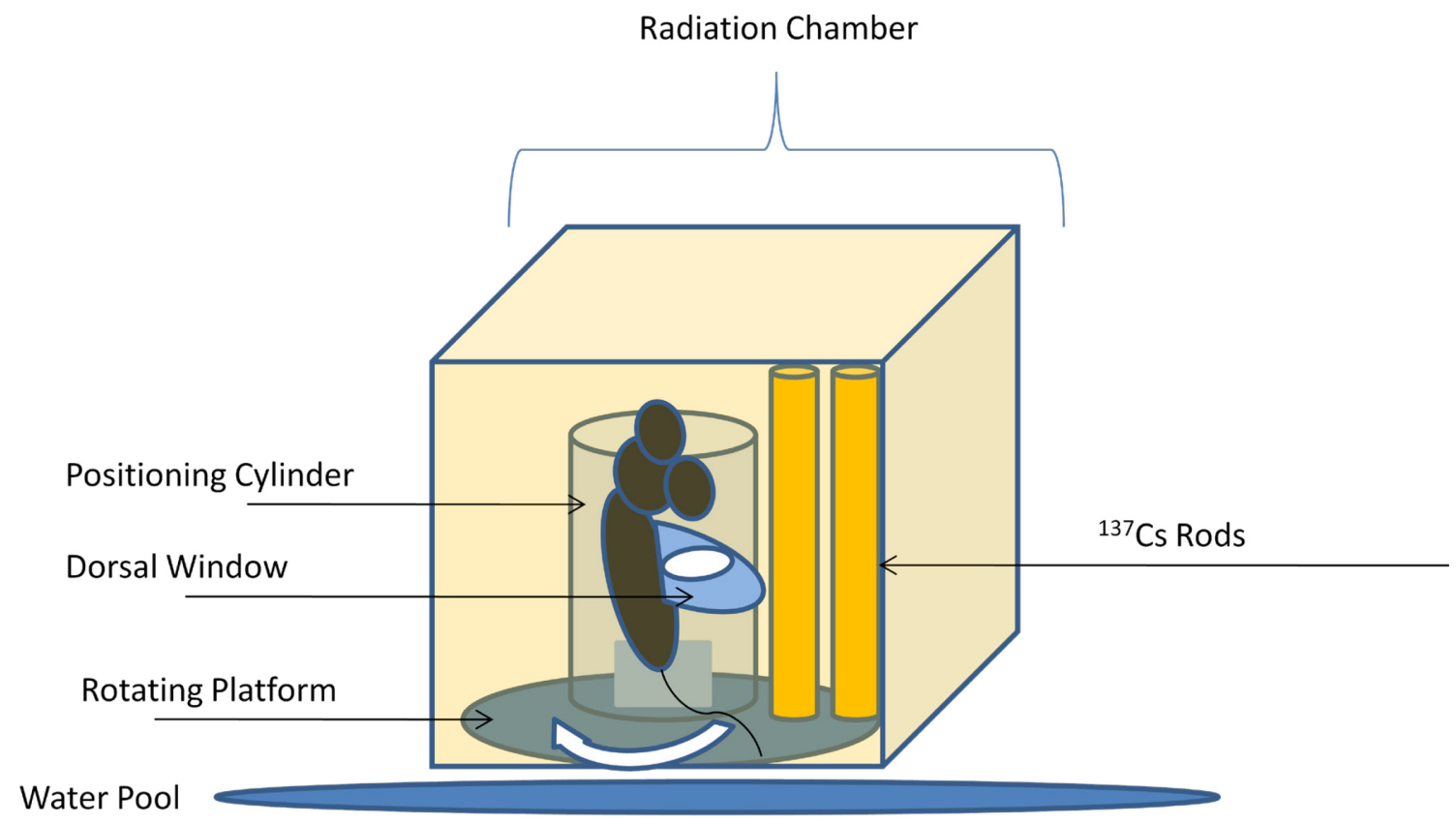

Figure 2-2 Delivering TBI for Imaging Studies.

Window bearing anesthetized mice were irradiated individually on a rotating platform without shielding. The dose rate was approximately $7 \mathrm{~Gy} / \mathrm{min}$ and a total dose of $6 \mathrm{~Gy}$ was delivered. 


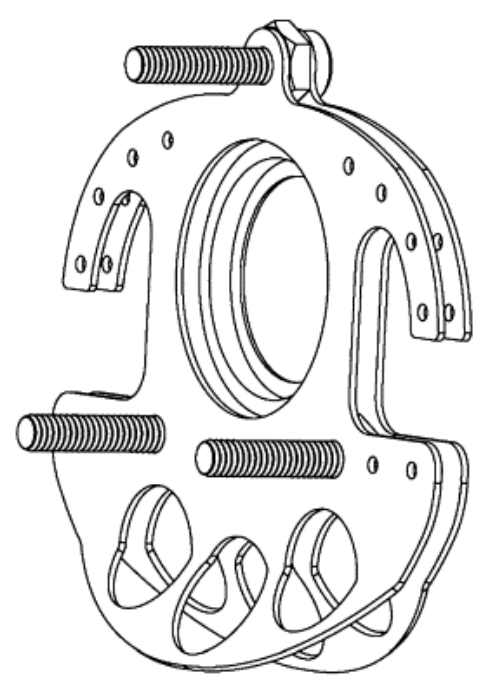

Figure 2-3 Dorsal Skinfold Window Apparatus.

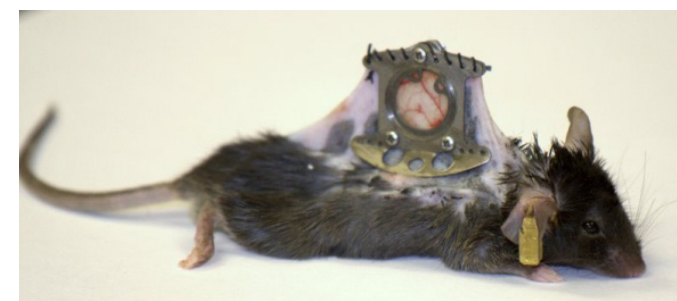

Figure 2-4 Mouse Fixed with DSFC.

C57/BL6 mice were surgically fixed with the dorsal window and allowed to recover for 3 days prior to further manipulation. 


\section{Intravital Microscopy}

Window-bearing animals were anesthetized and received an intra-orbital injection of rhodamine $6 \mathrm{G}(0.4 \mathrm{mg} / \mathrm{kg}$ body weight $)$ for leukocyte visualization. All experiments were carried out using an industrial grade microscope (Nikon MM-11) with two light sources, bright-field (Opti-Quip $75 \mathrm{~W}$ xenon) and fluorescent (Nikon $150 \mathrm{~W}$ mercury). Experiments were viewed on a video monitor and recorded for off-line processing. The video images were analyzed off-line using MetaMorph software (Universal Imaging Co.). Rolling and Adhered (stationary for $>30$ seconds) leukocytes were quantified and recorded.

\section{Induction of Vascular Injury}

The snap ring and glass window covering the dermal vasculature was removed. The tissue was slightly swabbed with sterile cotton tipped applicator. Immediately prior to injury induction animals were injected with Rhodamine 6G so that leukocytes and platelets would be visible microscopically. The vascular injury was induced by "nicking" a vessel with a sterile 30-gauage needle. Once the nick was induced, a new cover slip was placed over the tissue and secured with a metal snap ring (Figure 2-5). Animals were immediately placed on the imaging platform for intra-vital imaging. Animals were injured (vascular injury) every 24 hours beginning 24 hours after TBI to establish model parameters (Figure 2-6).

\section{Blood Collection}

Window-bearing animals were anesthetized with ketamine/xylazine (87:13, i.p.) and blood was collected via cardiac puncture into plasma separator tubes containing lithium heparin. Blood was gently mixed by inversion and immediately placed on ice until analysis and processing. Processed plasma (centrifuged at 10,000 x g 10 min $4^{\circ} \mathrm{C}$ ) was aliquoted and stored at $-80^{\circ} \mathrm{C}$ until cytokine/chemokine analysis.

\section{CBC Analysis}

Complete blood analysis was performed on heparinized whole blood with a Beckman Coulter ${ }^{\circledR}$ Ac $\bullet T$ diff ${ }^{\mathrm{TM}}$ Analyzer as quickly as possible after collection $(<1$ hour).

\section{Cytokine/Chemokine Analysis}

Following CBC analysis, blood was processed and stored as described above. Cytokine concentrations in the plasma of animals from all groups was analyzed using the Luminex platform. 


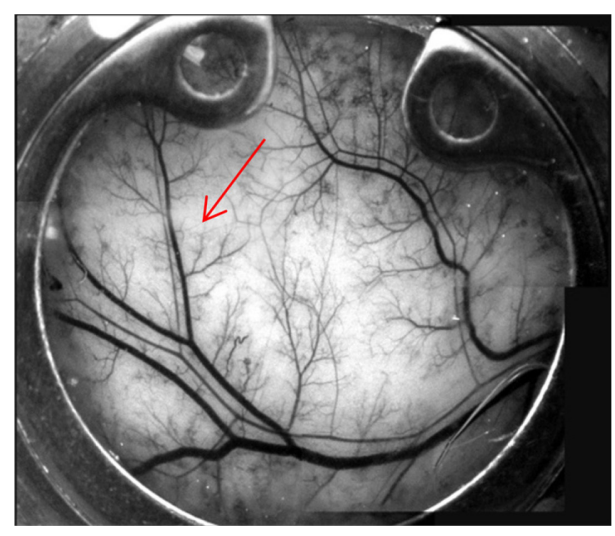

Figure 2-5 Low Magnification Image of Dermal Vasculature.

Low magnification view of the dermal vasculature following surgical implantation of DSFC. Red arrow indicates an ideal site for injury induction.

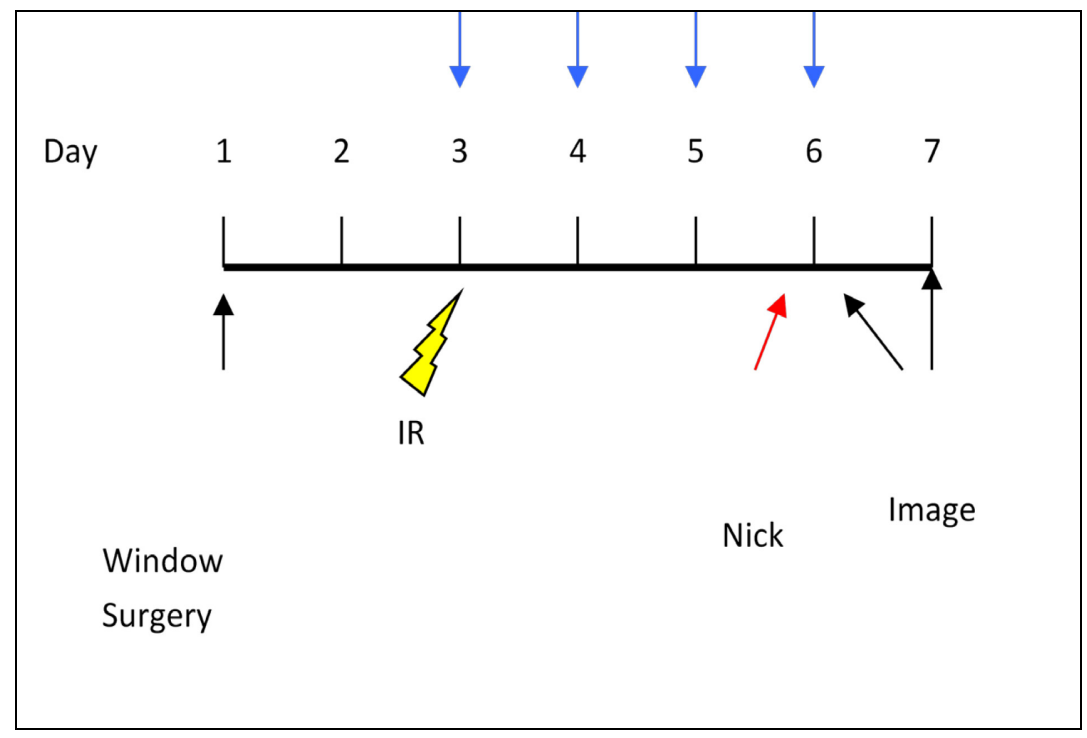

Figure 2-6 Imaging Study Timeline.

Window implantation occurred on study day 1 with radiation on day 3 . Daily treatments began 12 hours after radiation and continued through the end of the study. Vascular injury was induced on study day 6 , with imaging immediately following. A second image was collected 24 hours after injury (Study day 7) to evaluate flow restoration. 


\section{Data Analysis}

All data are expressed as mean plus or minus standard deviation. P values of $<$ 0.05 were considered statistically significant.

\section{RESULTS}

\section{Murine Survival}

The primary endpoint of this study was 30-day mortality. Animals treated with a single dose of KZ-41 26 hours after lethal total body irradiation showed an approximate $40 \%$ improvement in survival when compared with vehicle (saline) treated animals (Figure 2-7).

\section{Clot Formation}

The primary endpoint in our imaging model was clot formation. Initial clot in control (unirradiated animals) occurred within 2 minutes of injury (Figure 2-8A), and evidence of remodeling could be observed as early as 8 minutes after vascular injury. There was no difference in clot formation when vascular injury was induced 24 or 48 hours after TBI compared to control. There was no clot formation when vascular injury was induced 72 hours after TBI (Figure 2-8B). 96 hours after TBI animals demonstrated fragile vasculature and poor window quality which prevented consistent vascular injury and analysis (Table 2-1). Induction of vascular injury 72 hours after TBI was optimal for treatment evaluation in this model; therefore, all treatments were evaluated under this time scheme. In untreated animals injured 72 hours after TBI, no clot formed in 5 of 6 animals measured (Table 2-1). Animals receiving KZ-41 treatment every 24 hours beginning 12 hours after TBI, showed significant improvement in clot formation compared with untreated animals (Figure 2-8C), with clots forming in 7 out of the 8 animals measured (Table 2-1).

\section{Flow Restoration}

The restoration of flow in the injured vessel both upstream and downstream of the injury was analyzed 24 hours after vascular injury. TBI significantly decreased restoration of flow when vascular injury was induced 72 hours after radiation (1/6 TBO vs. 5/6 Control animals, Table 2-1). Three daily treatments with KZ-41 beginning 12 hours after TBI resulted in flow restoration in $43 \%$ of the treated animals $(3 / 7)$ compared with $17 \%$ of TBI alone animals, however, this improvement was not statistically significant. 


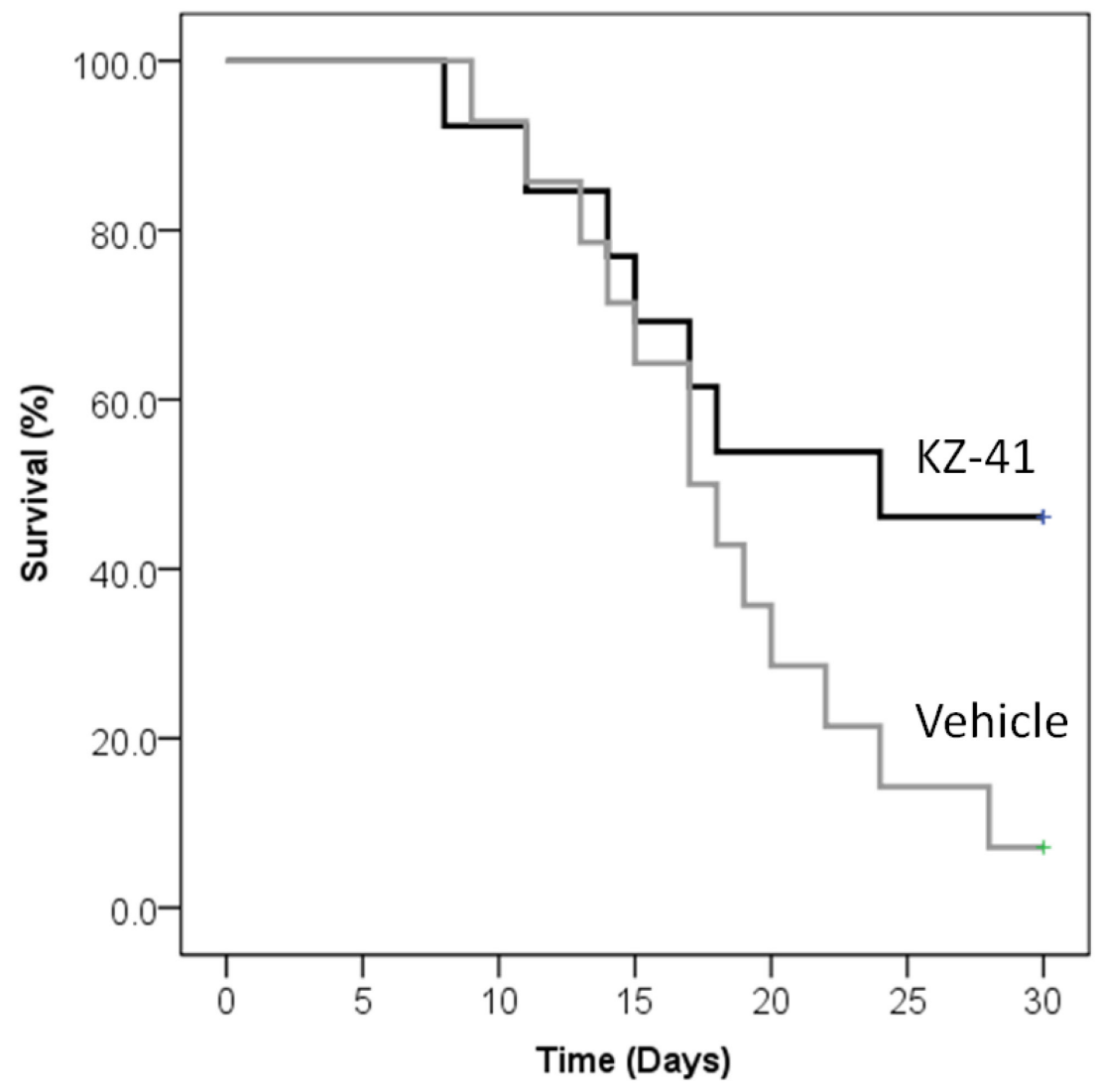

Figure 2-7 Effect of KZ-41 on Murine 30 Day Survival.

KZ-41 (100 mg/kg or Vehicle (normal saline) was administered once 25 hours after lethal total body irradiation $\left(\mathrm{LD}_{80 / 30}\right)$.

$\mathrm{n}=24-26$ per group

$\mathrm{p}<0.05$ 

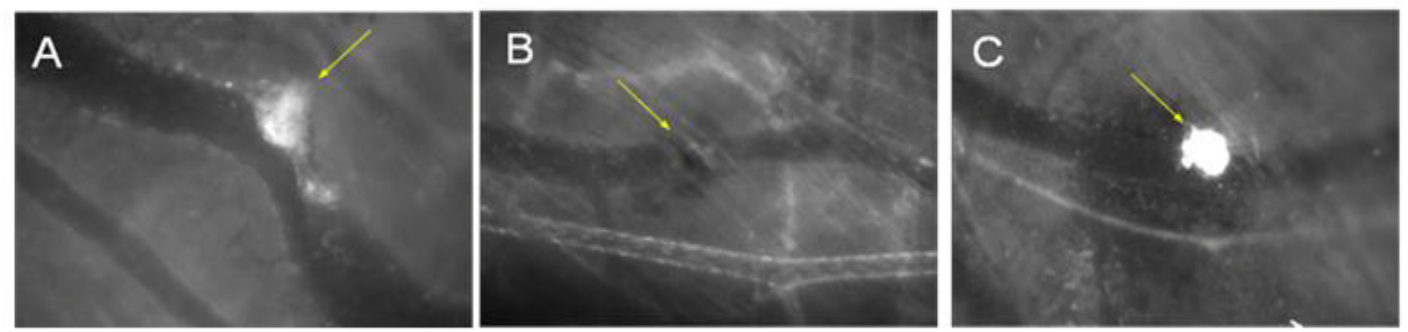

Figure 2-8 Effect of KZ-41 on Radiation-Induced Clotting Abnormalities.

Clot formation after vascular injury in (A) Unirradiated Controls (B) Irradiated + Vehicle (Normal Saline), and (C) Irradiated + KZ-41 (100 mg/kg).

Table 2-1 Effect of KZ-41 on Clot Formation.

\begin{tabular}{lll}
\hline Group & Clot & Flow \\
& Restoration & Restoration \\
\hline Unirradiated & $5 / 6(83 \%)$ & $5 / 6(83 \%)$ \\
Vehicle & $1 / 6(17 \%)^{*}$ & $1 / 6(17 \%)^{*}$ \\
KZ-41 & $7 / 8(88 \%)^{\#}$ & $3 / 7(43 \%)$ \\
\hline
\end{tabular}

Mice were irradiated (6 Gy TBI) and treated (vehicle was normal saline, $100 \mathrm{mg} / \mathrm{kg} \mathrm{KZ}$ 41 , s.q.) every 24 hours beginning 12 hours post TBI. Vascular injury was induced 3 days post TBI and cot formation was immediately assessed. Flow restoration was measured 24 hours following vascular injury.

*Unirradiated control vs. irradiated vehicle treated mice $(\mathrm{p}<0.05)$

\#KZ-41 vs. vehicle treated irradiated mice $(\mathrm{p}<0.05)$ 


\section{CBC Analysis}

Circulating platelet and leukocyte counts were measured in animals receiving TBI 72 hours prior to vascular injury. Blood was collected and analyzed at the completion of the study ( $96 \mathrm{hrs}$ post TBI). There was a significant decrease in both platelet and leukocyte numbers in the periphery (Figures 2-9, 2-10). Animals treated with KZ-41 did not have elevated platelet or leukocyte counts compared with untreated animals (Figures 2-9, 2-10).

\section{Cytokine Analysis}

Circulating $\mathrm{KC}$ and TNF- $\alpha$ concentrations were measured in animals receiving TBI 72 hours prior to vascular injury. Blood was collected and analyzed at the completion of the study ( $96 \mathrm{hrs}$ post TBI). There was a significant increase in circulating $\mathrm{KC}$ and TNF- $\alpha$ in animals receiving TBI alone compared to control animals. There was no difference in measured cytokine levels between KZ-41 and untreated animals (Figures 2-11, 2-12).

\section{DISCUSSION}

There is currently no small-molecule approved for administration after exposure to radiation doses causing acute radiation syndrome to improve survival. The primary causes of death in individuals suffering from the heme-syndrome of ARS are infection and hemorrhage. These individuals exhibit decreased levels of circulating leukocytes and platelets (pancytopenia). The complications present in patients affected by ARS at the lower heme doses are also present and problematic in higher ARS doses (GI and CNS); therefore, it is important to have in vivo models in place to evaluate and develop potential treatments for all doses of ARS.

We have demonstrated that KZ-41 significantly improves survival when administered as late as 26 hours after lethal total body radiation. KZ-41 is a novel small molecule with an attractive biopharmaceutical profile which makes it an ideal drug candidate. Although the survival study met the efficacy endpoint for ARS, we wanted to further investigate the mechanisms by which KZ-41 was exerting its effect, particularly in the setting of combined vascular injury, while simultaneously addressing an unmet need for combined injury models.

The described combined injury model was optimized using 6 Gy Total Body Irradiation coupled with vascular injury on day 3 post TBI (Figure 2-6). Complete blood analysis, cytokine measurements, and other parameters may also be studied in the same animals as the imaging when coupled with careful interpretation. Imaging endpoints in our study were clot formation and restoration of flow, however, leukocyte activity and vessel diameter at the site of injury and in adjacent (irradiated only) vessels as well as vessel permeability in uninjured vessels may also be studied. Under the conditions 


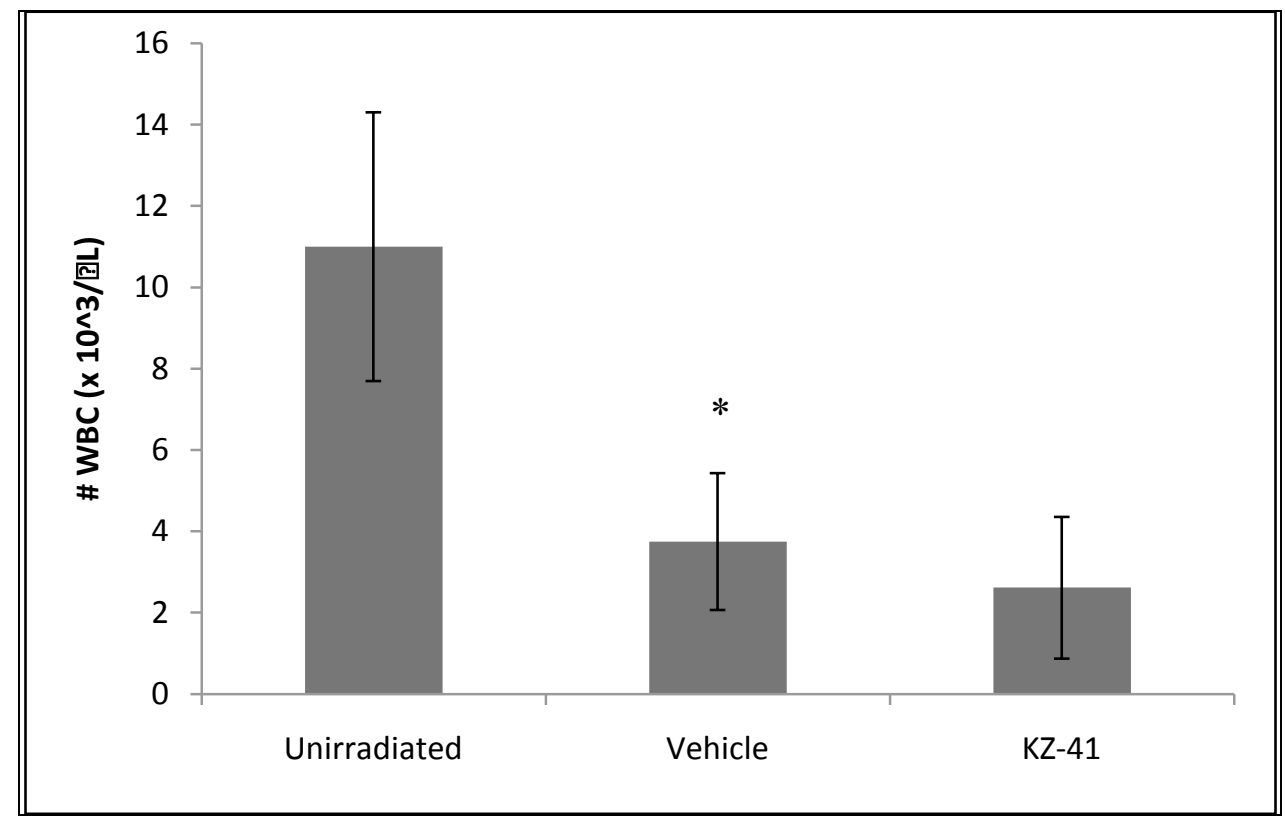

Figure 2-9 Leukocyte Counts 4 Days post TBI.

Blood was collected from window bearing mice on study day 7 and analyzed for circulating leukocytes.

*Unirradiated control vs. irradiated vehicle treated mice $(\mathrm{p}<0.05)$ 


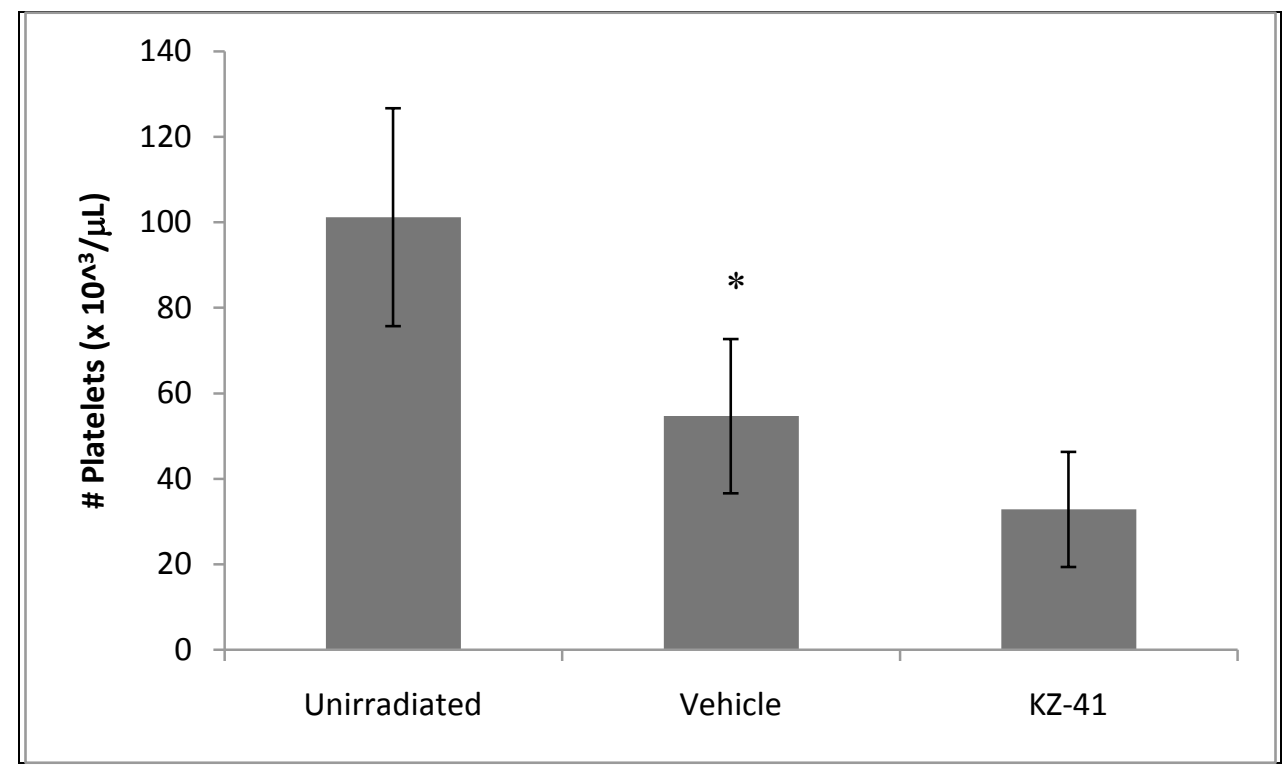

Figure 2-10 Platelet Counts 4 Days post TBI.

Blood was collected from window bearing mice on study day 7 and was analyzed for circulating platelet counts.

*Unirradiated control vs. irradiated vehicle treated mice $(\mathrm{p}<0.05)$ 


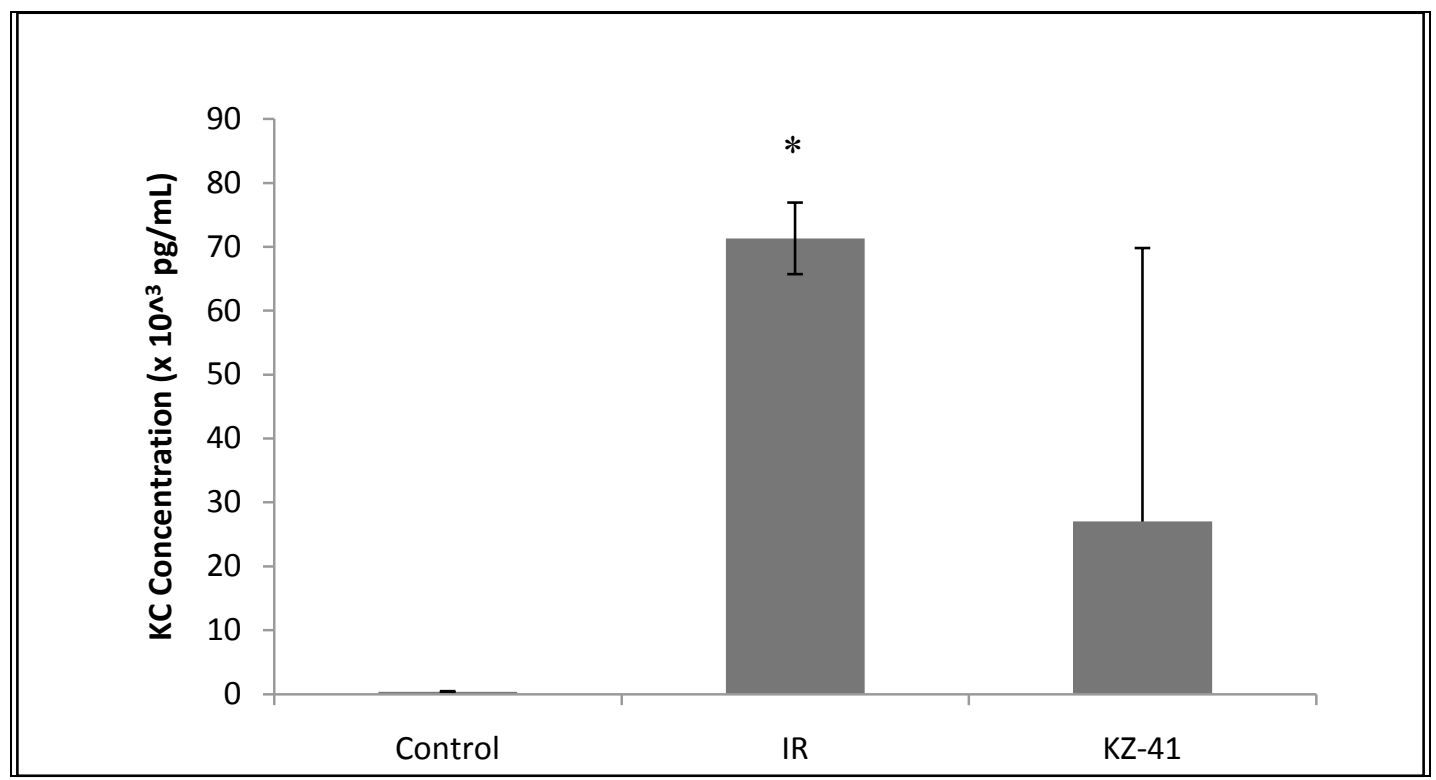

Figure 2-11 KC Concentration 4 Days post TBI.

Blood was collected from window bearing mice on study day 7 and was analyzed for circulating $\mathrm{KC}$ concentration.

*Unirradiated control vs. irradiated vehicle treated $(I R)$ mice $(p<0.05)$ 


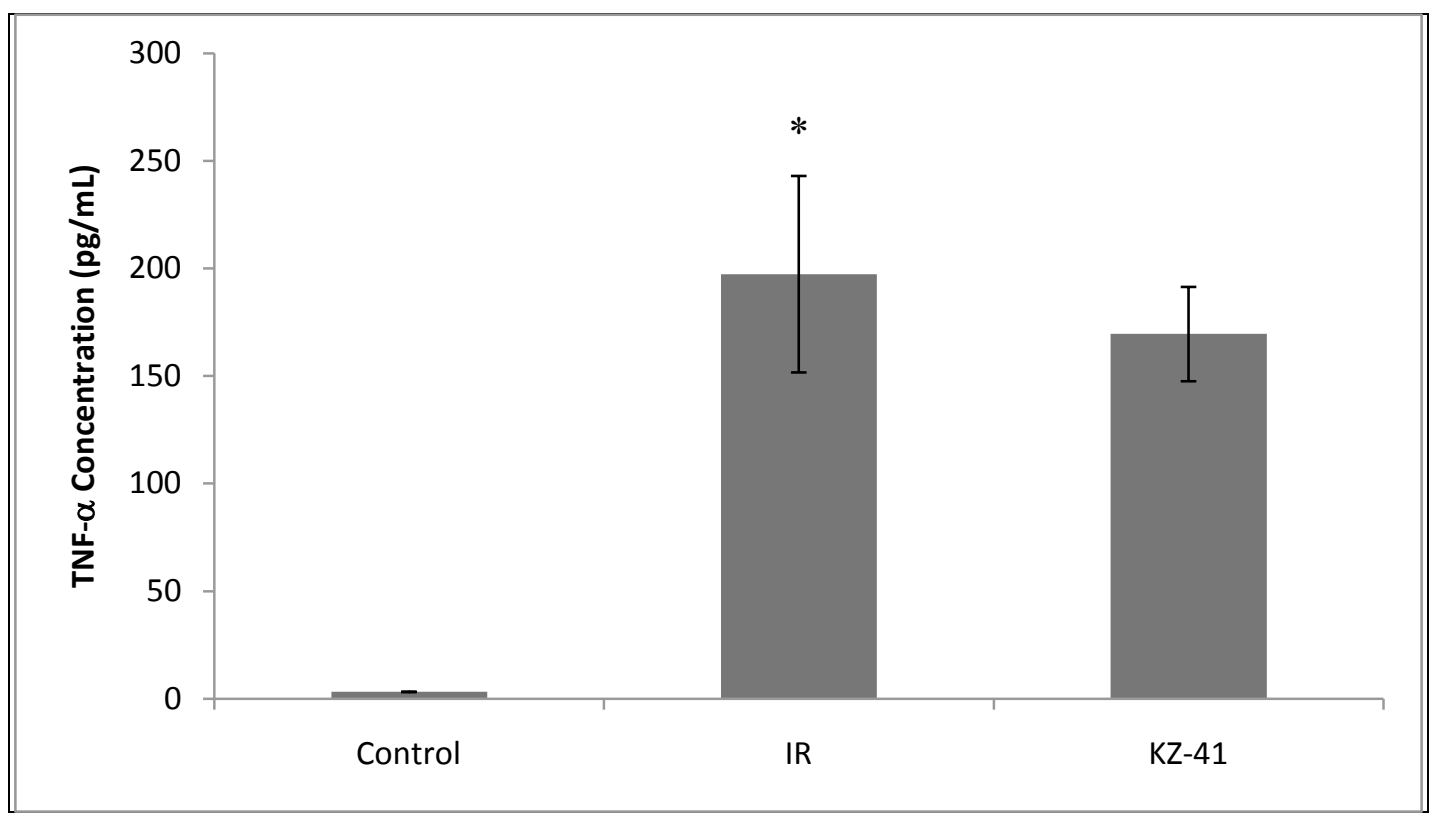

Figure 2-12 TNF- $\alpha$ Concentration 4 Days post TBI.

Blood was collected from window bearing mice on study day 7 and was analyzed for circulating TNF- $\alpha$ concentration.

*Unirradiated control vs. irradiated vehicle treated (IR) mice $(\mathrm{p}<0.05)$ 
described, there was a significant reduction in the formation of a clot immediately following vascular injury and restoration of vascular flow in the injured vessel compared with unirradiated control animals. The novel radiomitigant KZ-41 significantly restored clot formation, and improved the incidence of flow restoration although not to significance when first administered 12 hours following lethal TBI. The effect of KZ-41 on clot formation in this model was not related to circulating platelet numbers as evidenced to similar platelet counts as untreated irradiated animals. We hypothesize that the effect demonstrated in the imaging model on clot formation is due to platelet function, and further analysis is planned to test these hypothesis.

Although this model has proved invaluable in our drug evaluation studies, there are limitations that should be considered. The surgery and subsequent radiation the animals receive places them in an extremely fragile state, and in our opinion prevents escalation of the dose beyond 6 Gy outside of acute endpoints. Also due to the physical burden of the window, one must evaluate cytokine and chemokine parameters carefully. The model that we have developed and described is a unique, versatile model to study combined radiation and vascular injury. This is the first radiation combined injury model described which allows investigation of vascular response in the setting of ARS [6]. This model may be expanded or condensed based on the specific aims of the project. 


\section{CHAPTER 3. IN VITRO MECHANISM}

\section{INTRODUCTION}

Damage induced by ionizing radiation encompasses two primary categories: initial damage and bystander damage (or non-targeted effects). The two causes of initial damage from directly irradiating cells is the charged particles that directly interact with cellular structures (direct action), and free radical generation which induces double strand breaks and oxidative stress (indirect action). Signals are produced by the damaged cells and are transferred through media and to a lesser extent through gap junctions [31]. The damage caused to unirradiated cells through these signals is a phenomenon known as the bystander effect.

In the setting of ARS understanding the bystander effect is of particular interest when discussing the efficacy elicited by a radiomitigant. By definition, a radiomitigant is administered after the radiation event. Therefore, for a clear understanding of the mechanism of action of a radiomitigating compound one must investigate the downstream events that occur after the initial "trigger" radiation event. A recently published study investigated the transcriptomes of both directly irradiated and bystander (via media transfer) cells and concluded that they are very similar, suggesting a propagation of injury phenomenon [32].

Various groups have investigated the bystander effect via directly irradiating a single cell(s) by using micro beam radiation and observing the effects on the surrounding cells [33]. This experimental setup has primarily focused on the role gap junctions play in the bystander effect phenomenon. Investigations into bystander signaling using mixed populations (directly irradiated and unirradiated) of cells as well as multiple cell types have been also been conducted. The important role of death receptor signaling in the bystander effect has also been demonstrated using medium transfer experiments [32, 34, 35].

Although radiation-induced bystander effects have been demonstrated for a variety of biological endpoints, we focus on effector caspase activation as an indicator of apoptosis and bystander effect. Extracellular-initiated apoptosis signaling executes down one of two primary pathways. Mitochondrial independent apoptosis is mediated by death-receptor ligand, activation of Caspase- 8 then Caspase-3, and ending in apoptosis. Mitochondrial dependent apoptosis is mediated by Bax, Bak, Bcl-2, Cytrchrome C release, activation of Caspase-9 then Caspase-3, and ending in apoptosis. The final step (Caspase-3 activation) prior to apoptosis is common to both pathways, and is thus an attractive surrogate marker for measuring apoptosis. 


\section{MATERIALS AND METHODS}

\section{Cell Line}

Human monocytic lymphoma cells (U937) stably transfected with a gentamycin resistant gene were purchased from ATCC. Cells were grown in RPMI 1640 media supplemented with $10 \%$ and $50 \mu \mathrm{g} / \mathrm{mL}$ concentration of gentamycin in a $37^{\circ} \mathrm{C}$ incubator with $5 \% \mathrm{CO}_{2}$.

\section{Irradiation}

Cells received a dose of 7 Gy from a ${ }^{137} \mathrm{Cs}$ source at a dose rate of approximately $8 \mathrm{~Gy} /$ minute on a rotating plate to ensure uniform exposure.

\section{Treatment}

U937 cells were divided into two different treatment groups as discussed below. Treatments were dissolved into low serum (1\%) medium at a concentration of $100 \mathrm{mM}$ and serially diluted to the desired concentration. All wells were treated in triplicate.

\section{Directly Irradiated Cells}

Cells were plated in MultiScreen ${ }_{\text {HTS }}$-PCF filter plates (Millipore, Billerica, MA) approximately 18 hours prior to irradiation in growth medium containing $1 \%$ FBS and 50 $\mu \mathrm{g} / \mathrm{mL}$ at a concentration of $1 \times 10^{6}$ cells per $\mathrm{mL}$. Cells were incubated under serum starved conditions at $37^{\circ} \mathrm{C}$ with $5 \% \mathrm{CO}_{2}$. All treatments (including vehicle) were conducted immediately (approximately 0.5 hours) prior to irradiation. Treatments were dissolved in fresh medium containing $1 \% \mathrm{FBS}$ and $50 \mu \mathrm{g} / \mathrm{mL}$ gentamycin. Cells were incubated at $37^{\circ} \mathrm{C}$ with $5 \% \mathrm{CO}_{2}$ for various lengths of time following radiation at which time cells were analyzed for markers of damage, repair, and apoptosis.

\section{Bystander Effect Cells}

Actively growing (bystander) cells were plated in MultiScreen HTS $_{\text {-PCF filter }}$ plates (Millipore, Billerica, MA) in growth medium at a concentration of $1 \times 10^{6}$ cells per $\mathrm{mL}$. Growth medium was removed via vacuum filtration. Treatments were dissolved in conditioned medium obtained after a 24 hour incubation post irradiation from directly irradiated cells. Conditioned medium $+/$ - treatments were then placed on the actively growing unirradiated cells and gently mixed for 1 minute on an orbital plate shaker. Cells were incubated at $37^{\circ} \mathrm{C}$ with $5 \% \mathrm{CO}_{2}$ for an additional 24 hours (referred to as 2424 ) at which time cells were analyzed for markers of damage, repair, and apoptosis. 


\section{Apoptosis}

Markers of apoptosis and DNA damage in the samples were measured in cell lysate per manufacturer's protocol using the Beadlyte ${ }^{\circledR}$ 3-Plex Apoptosis Signaling Kit and the pH2A.X Beadmate ${ }^{\circledR}$ (Millipore, St. Charles, MO) paired with the Luminex ${ }^{\circledR}$ $200^{\mathrm{TM}}$ System (Luminex Corporation, Austin, TX). Controls and samples $(50 \mu \mathrm{L})$ were mixed with $25 \mu \mathrm{L}$ of bead solution (fluorescent beads conjugated to antibodies for Active Caspase-3, GAPDH, Cleaved PARP, and pH2A.X) and incubated overnight at $4^{\circ} \mathrm{C}$. Wells were washed and beads resuspended in assay buffer. Biotin-conjugated antibodies (anti-Active Caspase-3, GAPDH, Cleaved PARP, and pH2A.X) were added and incubated for 1.5 hours. Streptavidin-Phycoerythrin was added and incubated 30 minutes. Unbound reagents were removed and fluorescence intensity reported as the median of 100 beads per analyte in each well. Median Fluorescent Intensity (MFI) values are reported as the $\%$ change from baseline.

\section{DNA Damage}

Millipore pH2A.X Beadmate ${ }^{\circledR}$ multiplexed with the Millipore Beadlyte ${ }^{\circledR}$ 3-Plex Apoptosis Signaling Kit (above) coupled with the Luminex platform.

\section{Cell Death}

Flow Cytometry was coupled with LIVE/DEAD® Cell Vitality Assay Kit with C12-Resazurin and SYTOX ${ }^{\circledR}$ green stain to evaluate metabolically active (live), metabolically inactive, and dead cells per manufacturer's protocol (Molecular Probes). Cells in suspension were incubated with $500 \mathrm{nM} \mathrm{C12-Resazurin} \mathrm{and} 10 \mathrm{nM} \mathrm{SYTOX}$ Green dye at $37^{\circ} \mathrm{C}$ for 15 minutes in the presence of $5 \% \mathrm{CO} 2$. Cell-dye mixtures were diluted and kept on ice until analysis by flow cytometry with fluorescence emission at $539 \mathrm{~nm}$ and $575 \mathrm{~nm}$. C12-Resazurin is reduced to red-fluorescent C12-Resorufin in cells that are metabolically active (delineated as live cells from this point forward). Dead cells with compromised plasma membranes will uptake the green-fluorescent nucleic acid stain SYTOX ${ }^{\circledR}$ Green dye, emitting green fluorescence. Metabolically inactive cells emit reduced red-fluorescence due to their reduced metabolic rate, but will exclude the green dye because they have intact membranes.

\section{Death Receptor Ligand Quantification}

Media samples were collected and stored at $-80^{\circ} \mathrm{C}$ until analysis with Beadlyte ${ }^{\circledR}$ multiplex cytokine analysis kit (Millipore, St. Charles, MO) paired with the Luminex ${ }^{\circledR}$ $200^{\mathrm{TM}}$ System. Standards and samples $(50 \mu \mathrm{L})$ were mixed with $25 \mu \mathrm{L}$ of bead solution and incubated overnight at $4^{\circ} \mathrm{C}$. Wells were washed and beads resuspended in assay buffer. Multi-cytokine biotinylated antibodies were added and incubated for 1.5 hours. Streptavidin-Phycoerythrin was added and incubated 30 minutes. Unbound reagents 
were removed and fluorescence intensity reported as the median of 75 beads per cytokine in each well. Concentrations were interpolated by fitting fluorescence intensity from unknowns to the standard curves.

\section{ROS Evaluation}

The ROS assay was carried out as previously described with minor modifications[36]. Dicholorofluorescindiacetate (DCFH-DA) dye was used for this assay. U937 cells were plated at a density of 150,000 cells/well in MultiScreen HTS $_{\text {-PCF }}$ filter plates (Millipore, Billerica, MA). Plates were then irradiated with a dose of $7 \mathrm{~Gy}$. After 24 hours, media was removed via vacuum filtration. Cells were then re-suspended in $100 \mathrm{uL}$ of $10 \mathrm{uM}$ DCFH-DA dye that had been reconstituted in DMSO and diluted in PBS. The plate was then covered in aluminum foil and incubated for 5 minutes at $37^{\circ} \mathrm{C}$. Dye was then removed via vacuum filtration. Cells were reconstituted in $200 \mathrm{uL}$ phenol red free RPMI. The plate was covered in foil and incubated for 30 minutes on a plate shaker at room temperature. Cells were then transferred to a black 96-well plate, and the plate was again covered in foil. The plate was incubated 30 minutes further at $37^{\circ} \mathrm{C}$. A Beckman Coulter DTX 880 Multimode Detector was used to read plate fluorescence with excitation and emission settings at 485 and $535 \mathrm{~nm}$ respectively.

\section{RESULTS}

\section{Direct Effect}

Apoptosis after Direct Irradiation Exposure. The effect of a single dose of ionizing radiation was evaluated on the U937 cell population over time. Activated Caspase-3 was measured at 1, 4, 24, 36, and 48 hours after IR (ionizing radiation). A bimodal increase was measured 24 and 48 hours after IR (Figure 3-1). No increase in phosphorylated histone $2 \mathrm{~A}(\mathrm{pH} 2 \mathrm{~A} . \mathrm{X})$ was observed in the time points that were measured (1, 4, 24, 36, and 48 hours) (Figure 3-2). Significant apoptotic cell death was verified 24 hours post IR (Figure 3-3). The effect of KZ-41 $(0.1,1,10$, and $100 \mu \mathrm{M}$ ) treatment 30 minutes prior to IR was investigated at both 24 hours and 48 hours after IR. There was no significant effect on active Caspase-3 levels at either 24 or 48 hours after IR (Figures 3-4, 3-5). There was no reduction in apoptotic cell death by KZ-41 24 hours after IR, which correlates with the Caspase-3 measurements (Figure 3-6).

Death Receptor Ligand after Direct Irradiation Exposure. Tumor necrosis factor alpha (TNF- $\alpha)$ was measured in the medium as a function of time $(1,4,24,36$, and 48 hours) following radiation exposure. TNF- $\alpha$ levels are below detection at 1 hour after IR exposure, however, may be detected as early as 4 hours post exposure. The maximum TNF- $\alpha$ concentration detected in our experiment was observed at 24 hours after exposure and was sustained at this elevated level through the last measured time point of 48 hours after exposure (Figure 3-7). KZ-41, when administered 30 minutes prior to irradiation, 


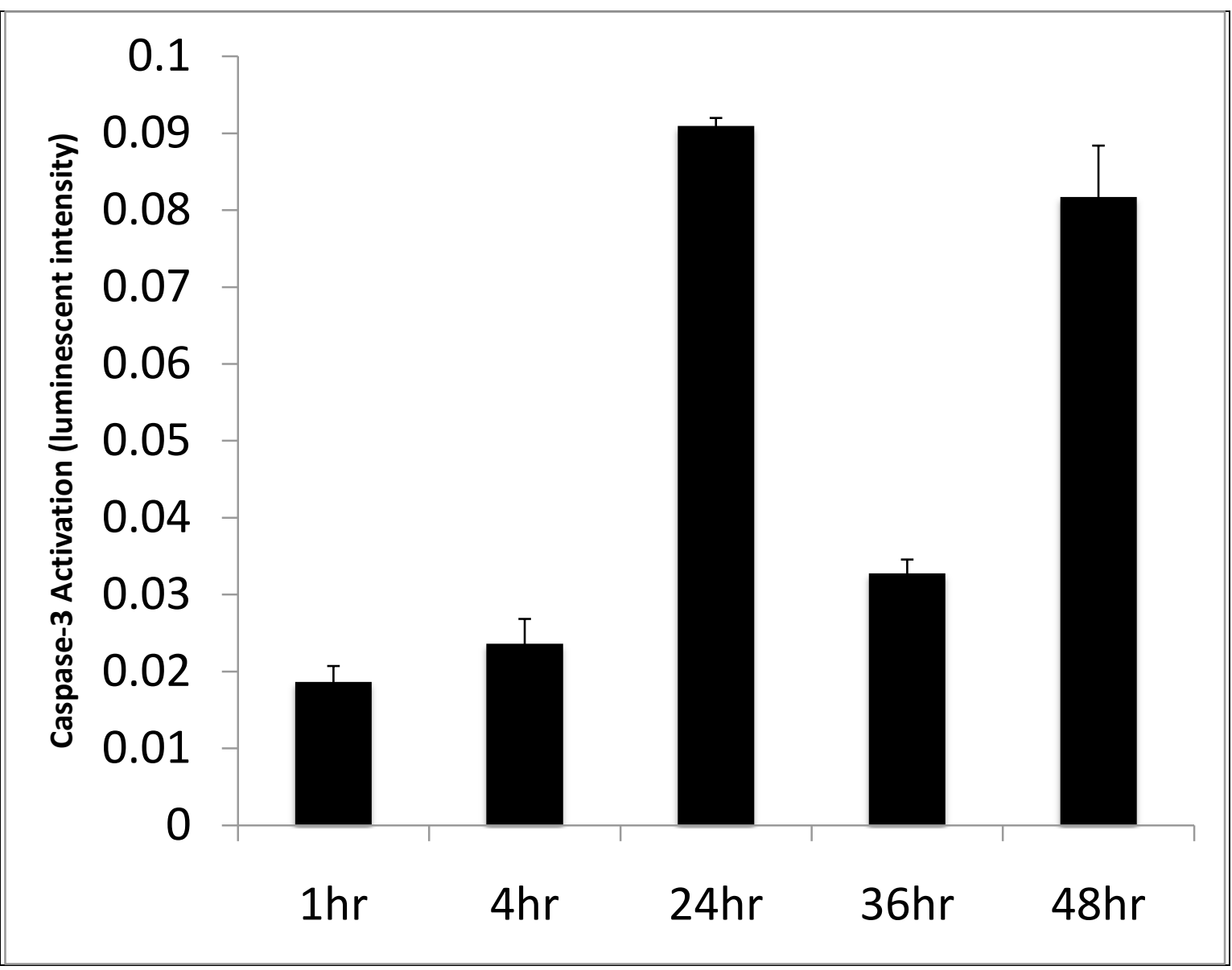

Figure 3-1 Caspase-3 Activation after 7 Gy IR.

U937 cell were irradiated (7 Gy) and Caspase-3 activation was measured using Luminex as a function of time as an indicator of apoptotic cell death. 


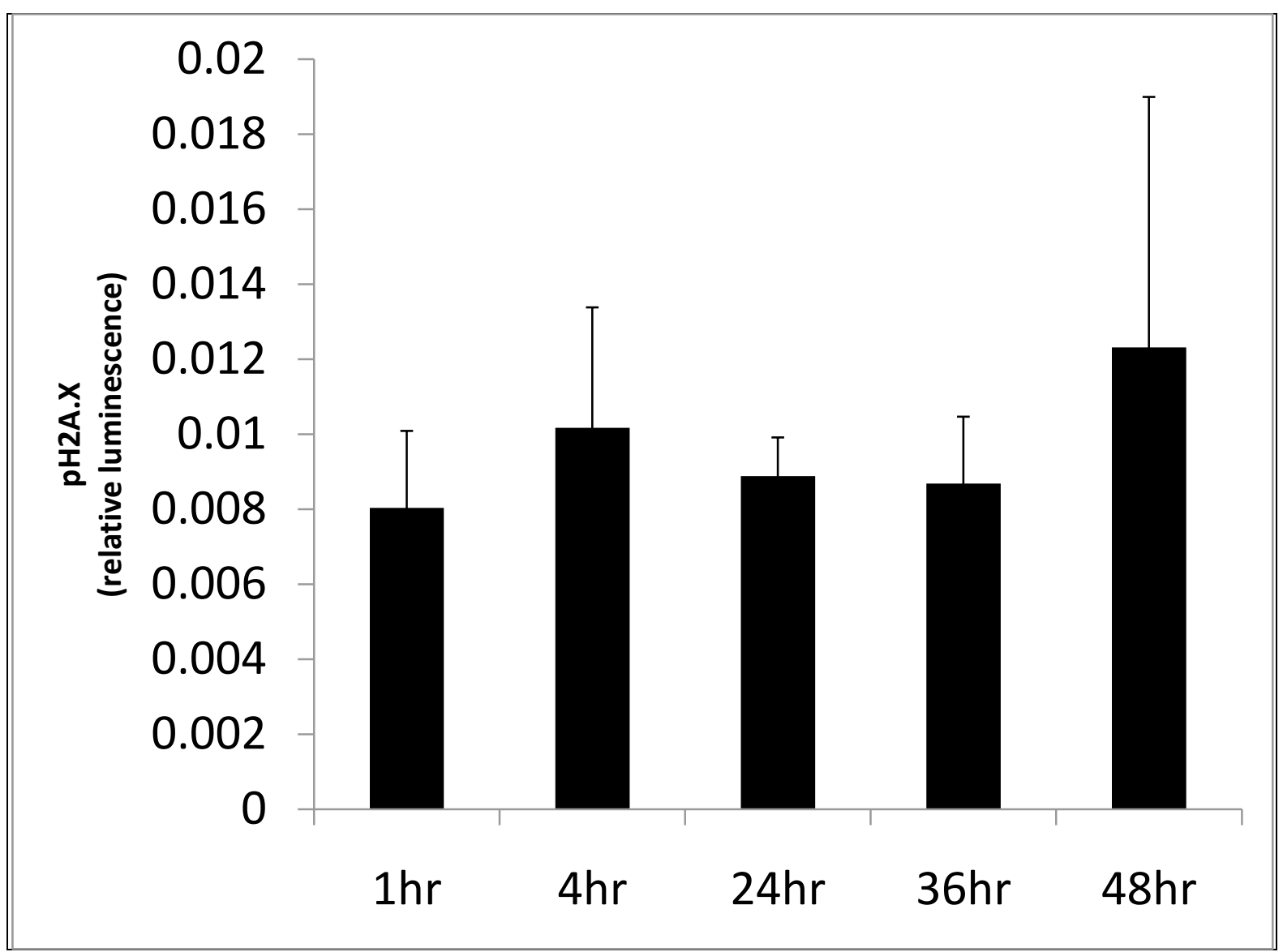

Figure 3-2 pH2A.X following 7 Gy IR.

U937 cell were irradiated (7 Gy) and DNA double strand breaks were measured as a function of time using Luminex. 


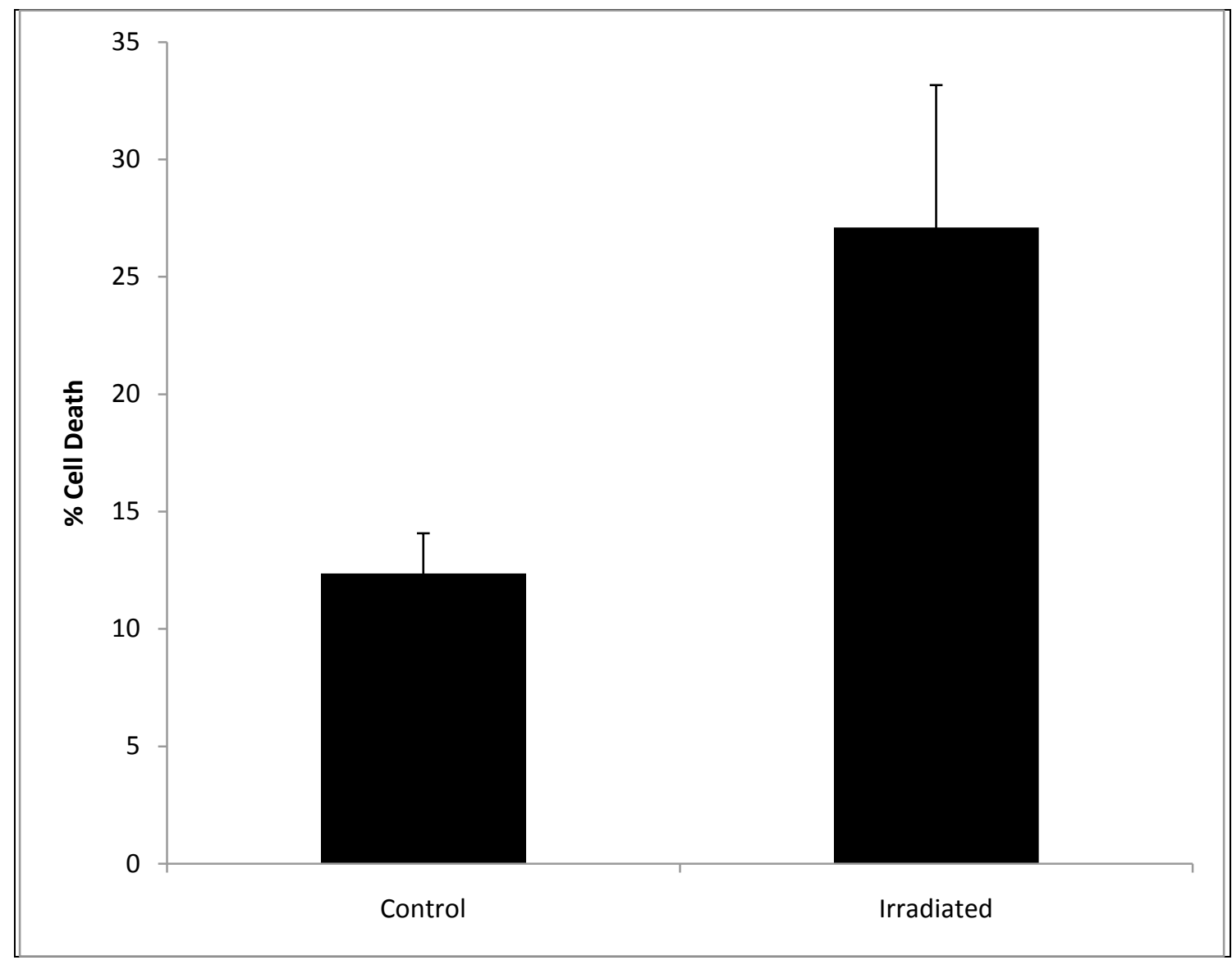

Figure 3-3 Apoptotic Cell Death 24 Hours following 7 Gy IR.

U937 cell were irradiated (7 Gy) and stained with propidium iodide 24 hours later. Apoptotic cell death was measured by flow cytometry. 


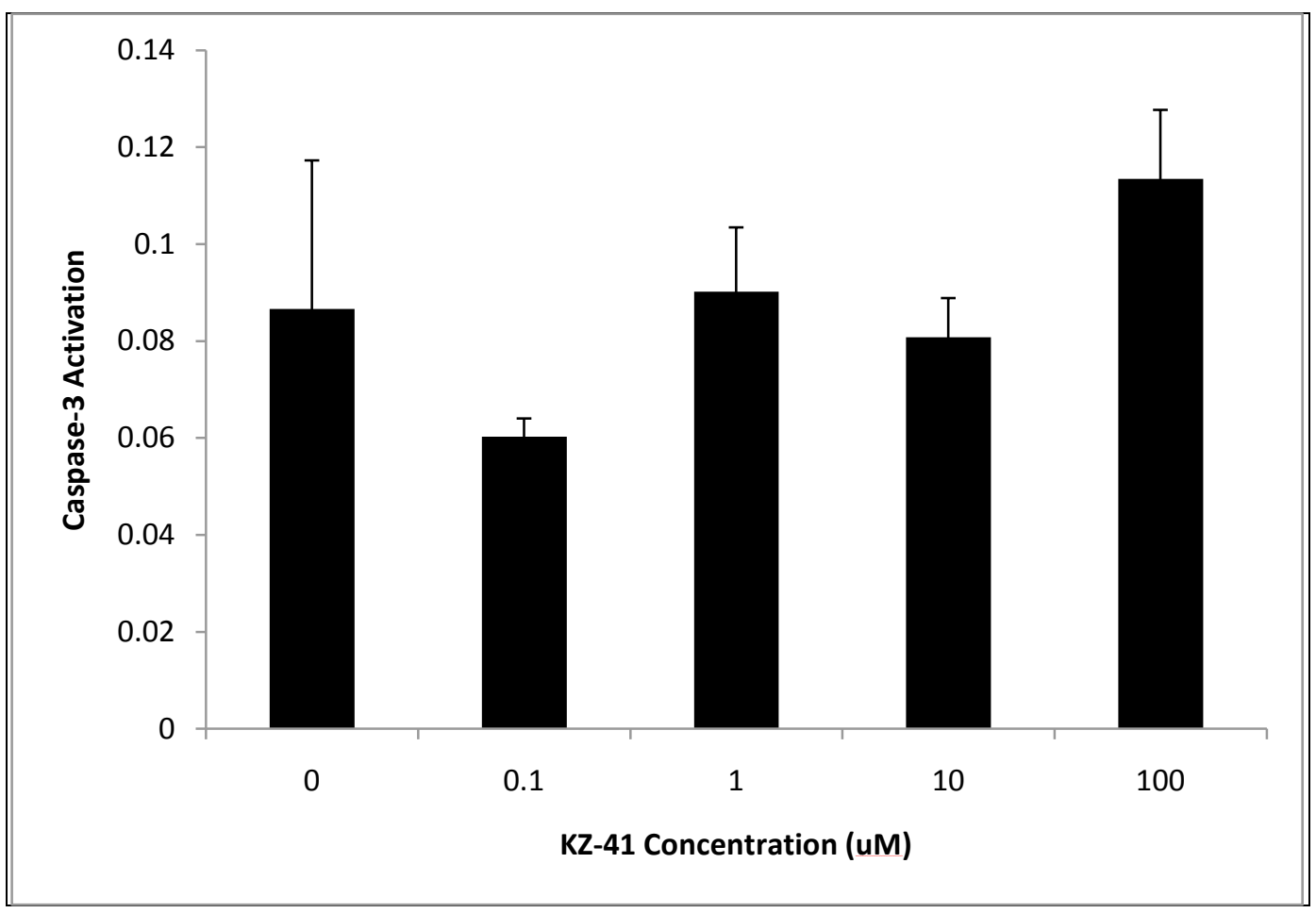

Figure 3-4 Effect of KZ-41 at 24 Hours on Radiation-Induced Direct Damage. U937 cells were treated with KZ-41 $(0-100 \mu \mathrm{M})$ and were immediately irradiated (7 Gy). 24 hours after radiation, Caspase- 3 was measured by Luminex. 


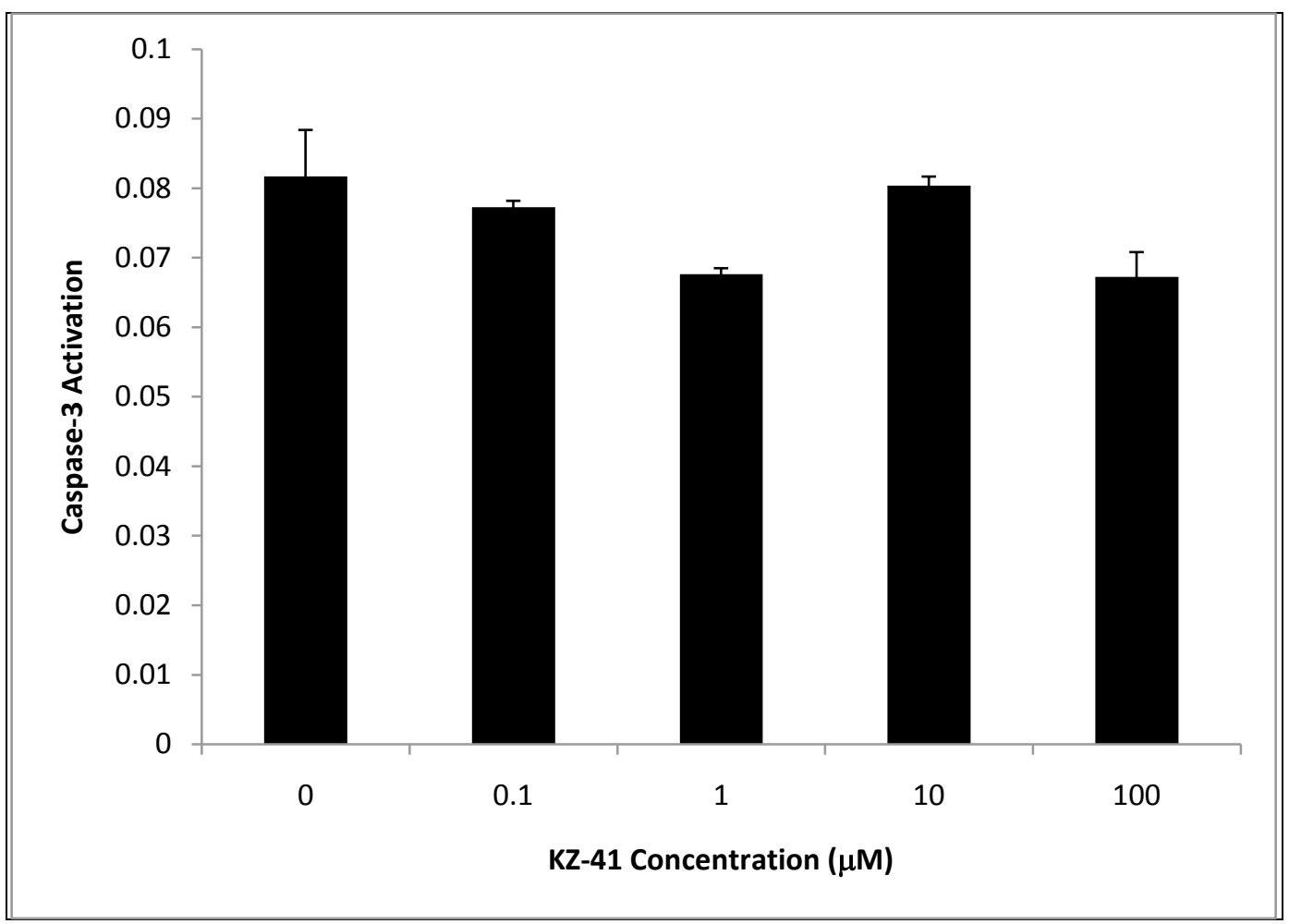

Figure 3-5 Effect of KZ-41 at 48 Hours on Radiation-Induced Caspase-3 Activation. U937 cells were treated with KZ-41 $(0-100 \mu \mathrm{M})$ and were immediately irradiated (7 Gy). 24 hours after radiation, Caspase-3 was measured by Luminex. 


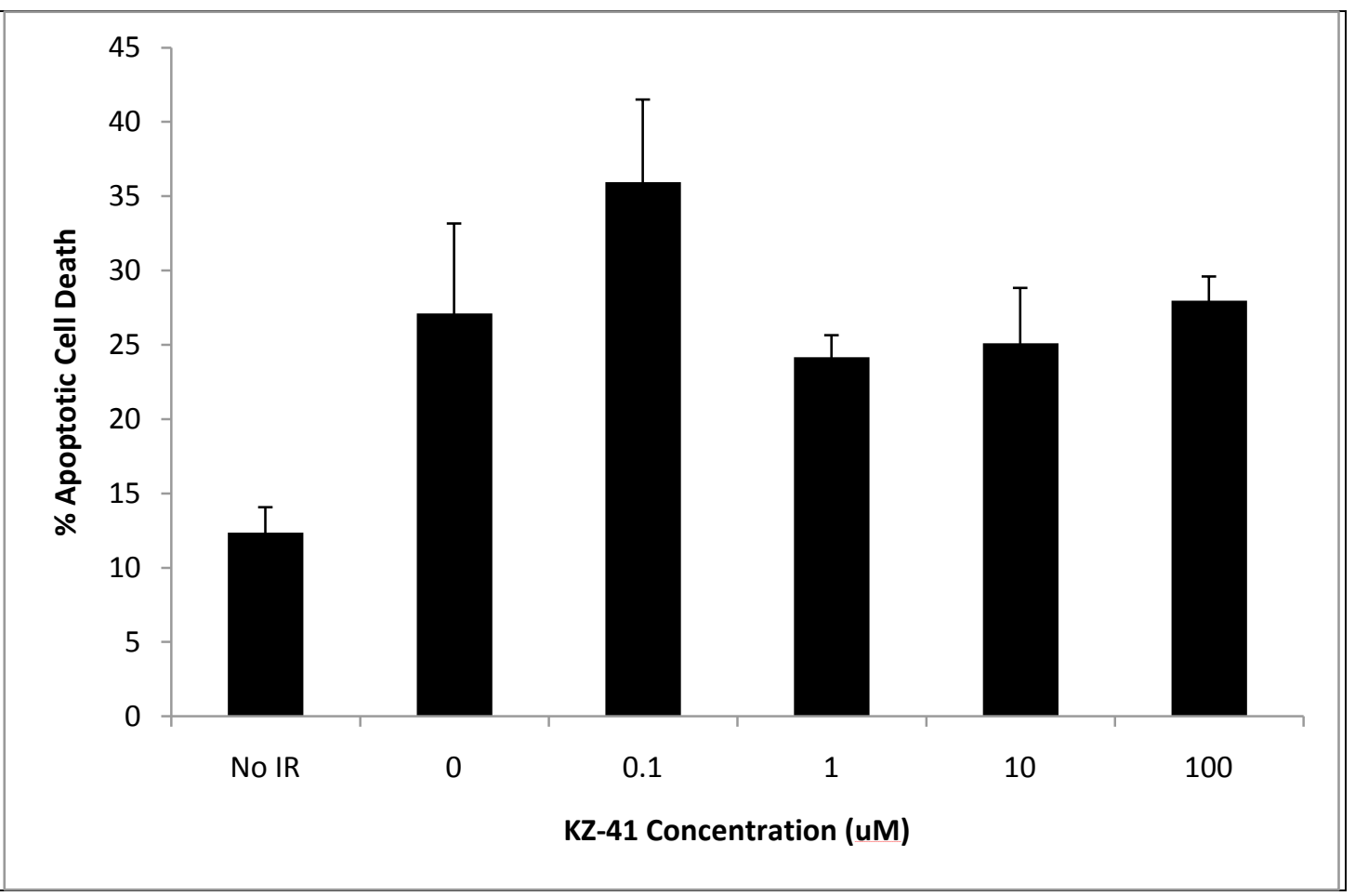

Figure 3-6 Effect of KZ-41 on Apoptotic Cell Death 24 Hours post Radiation Injury.

U937 cells were treated with KZ-41 $(0-100 \mu \mathrm{M})$ and were immediately irradiated (7 Gy). 24 hours after radiation, cells were stained with propidium iodide and apoptotic cell death was measured by flow cytometry. 
dose-dependently $(0.1-100 \mu \mathrm{M})$ reduced TNF- $\alpha$ concentration in the medium 48 hours after irradiation (Figure 3-8).

Reactive Oxygen Species. Reactive Oxygen Species (ROS) significantly increases 24 hours after irradiation, and KZ-41 $(1 \mu \mathrm{M})$ significantly reduces ROS (Figure 3-9).

\section{Bystander Effect}

Effect of Irradiation. The apoptotic effect 24 hours after a single transfer of conditioned medium (transferred 24 hours after IR), hereby referred to as 24-24, was measured via Caspase-3/7 activity to establish the possibility of a bystander effect in this model system. There is significant accumulation of Caspase-3 24 hours after IR (Figure 3-10), and degradation of Caspase- $3 / 7$ occurs when stored at $37^{\circ} \mathrm{C}$ compared with $-80^{\circ} \mathrm{C}$ for 24 hours (Figure 3-11). There are differences in total and soluble Caspase-3/7 at 2424 (Figures 3-11, 3-12), but not in cellular levels (Figure 3-13).

Effect of KZ-41 and Next Generation Compounds. KZ-41 caused a dosedependent (0.1-100 $\mu \mathrm{M})$ reduction in Caspase-3/7 in the 24-24 CM experimental setup (Figure 3-14). Next generation compounds (Figure 3-15) were analyzed at $1 \mu \mathrm{M}$ and compared to the efficacy of KZ-41 (Figure 3-16). KZ-157 demonstrated a significant improvement over KZ-41 in reducing Caspase-3/7 levels under this model system at a 1 $\mu \mathrm{M}$ concentration.

\section{DISCUSSION}

A single cell type in vitro model where both direct damage and the bystander effect may be monitored and used for drug efficacy testing in the setting of radiation injury has been presented. In the U937 cell line, we have shown that a radiation dose of 7 Gy is sufficient to induce apoptotic cell death under our experimental conditions. At 24 hours after IR, we were able to measure a significant increase in both Caspase- 3 and apoptotic cell death (Figures 3-1, 3-3), which is different from a previously published study who did not observe significant cell death prior to 30 hours after 7 Gy RI [36]. This group did not show the apoptosis data prior to 48 hours but they did mention their findings. One marked difference between the Kim group's findings and ours is serum starvation. The Kim group did not serum starve prior to radiation, and all of our data is reflective of serum starvation $(1 \% \mathrm{FBS})$ prior to radiation. This difference is an important one to note because serum starvation arrests cells in the G0/G1 phase in the cell cycle [15]. Approximately $40 \%$ of actively growing U937 cells are in S phase which is the most radiation resistant phase of the cell cycle $[37,38]$. Furthermore, although highly effective at promoting survival, we have demonstrated that our lead compound, KZ-41, has no effect on Caspase-3 or apoptosis when administered prior to radiation injury (Figures 34, 3-5, 3-6). Due to the nature of the therapeutic deficiency that we are $-\alpha$ after IR as a first step in developing a bystander effect model. We demonstrated a 


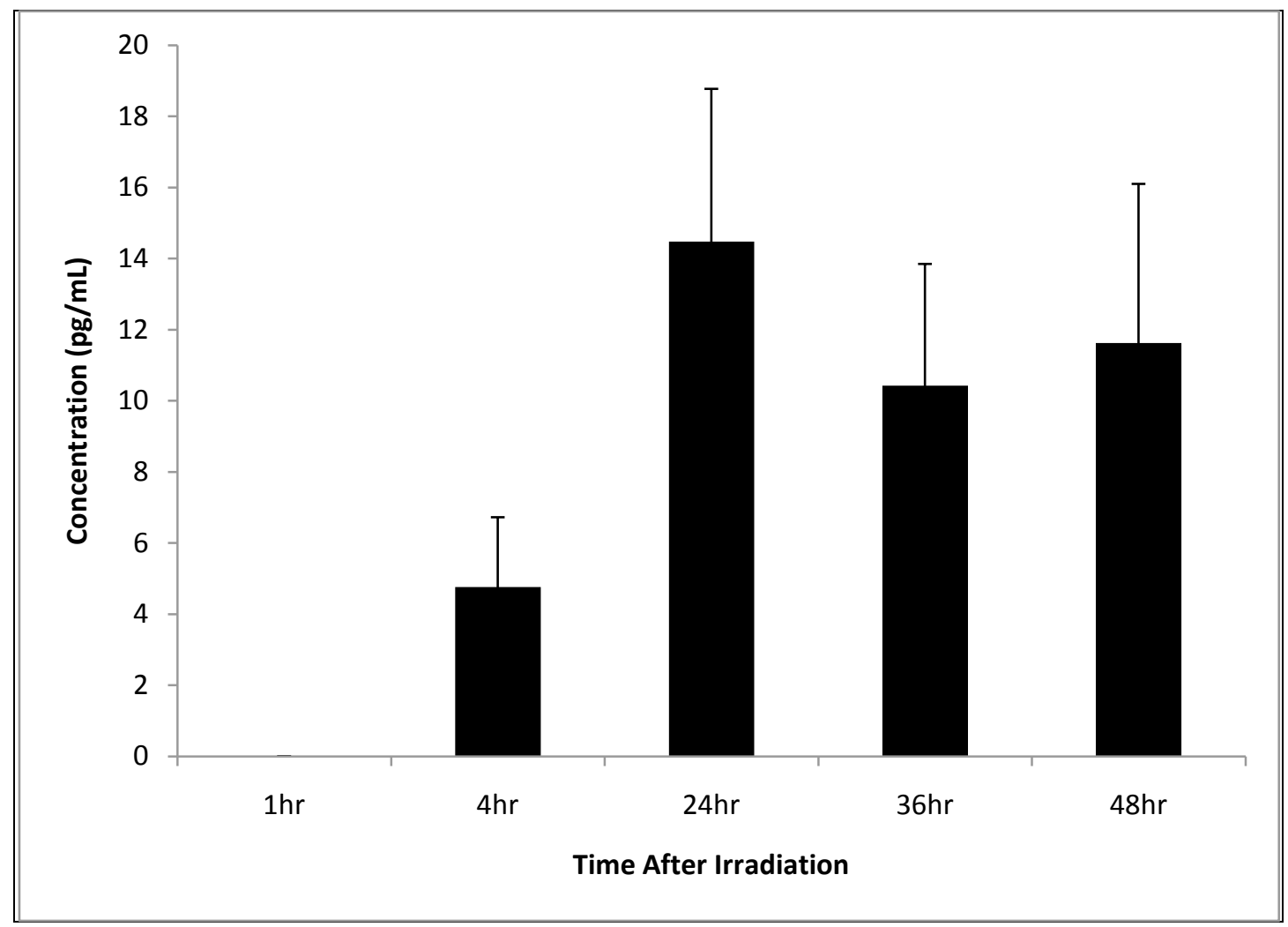

Figure 3-7 TNF- $\alpha$ Concentration following 7 Gy Radiation Exposure.

U937 cells were irradiated ( 7 Gy). TNF- $\alpha$ concentration in the medium was measured by Luminex as a function of time after irradiation. 


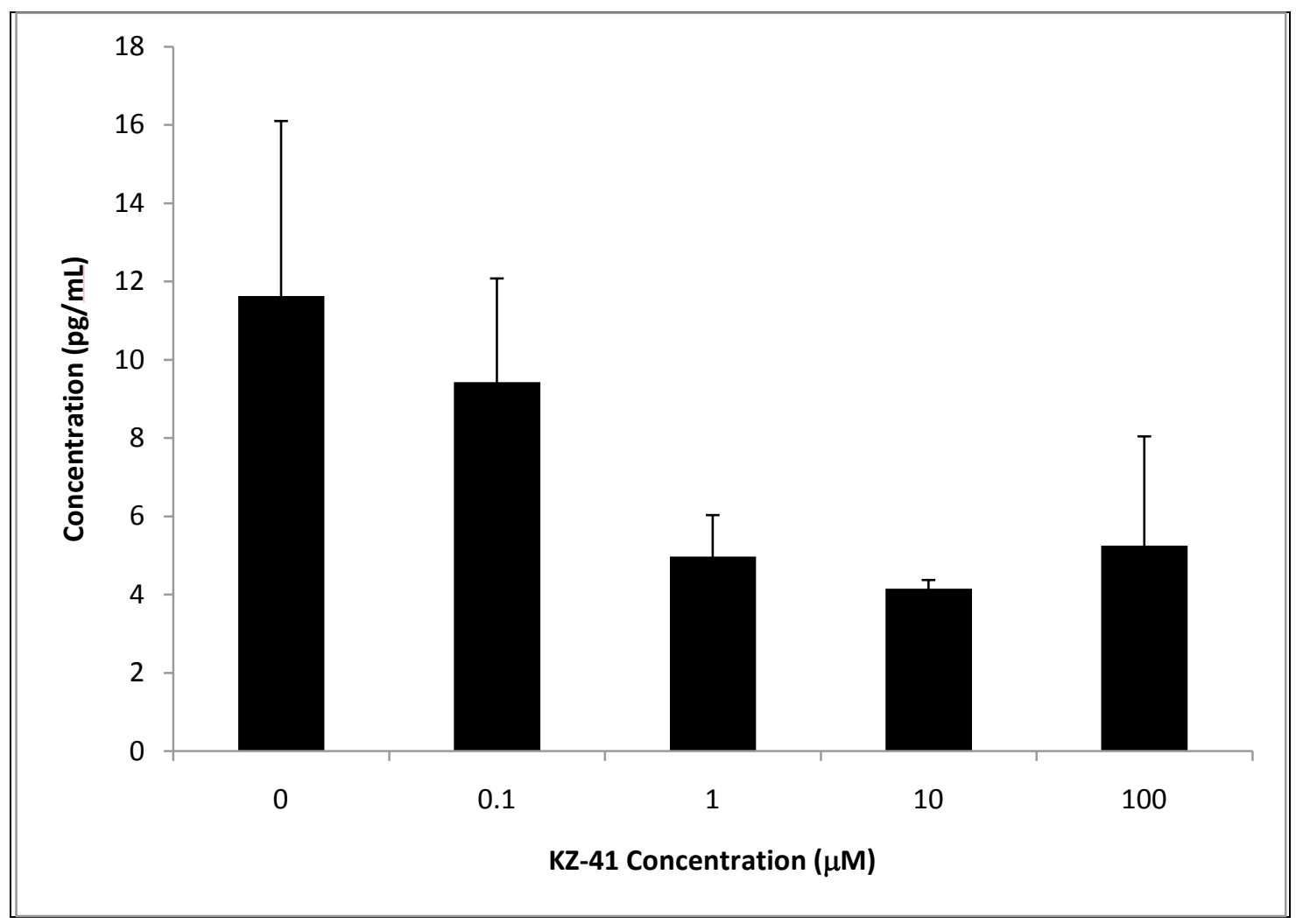

Figure 3-8 Effect of KZ-41 on TNF- $\alpha$ Production 48 Hours post Radiation.

U937 cells were treated with KZ-41 $(0-100 \mu \mathrm{M})$ and were immediately irradiated (7 Gy). 48 hours after radiation, TNF- $\alpha$ concentration in the medium was measured by Luminex. 


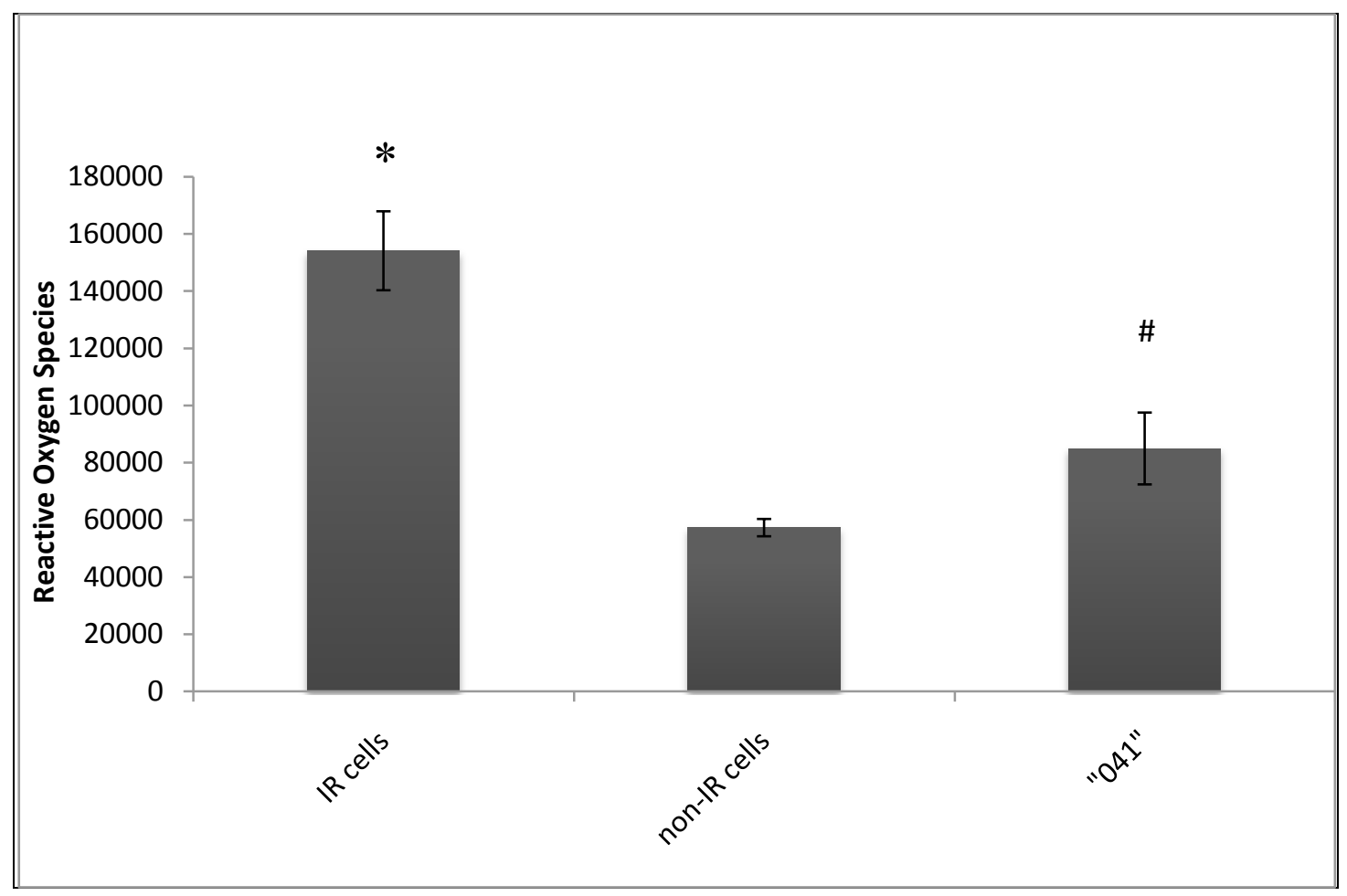

Figure 3-9 Effect of KZ-41 on ROS 24 Hours post Radiation.

U937 cells were treated with KZ-41 $(1 \mu \mathrm{M})$ immediately prior to irradiation (7 Gy). 24 hours after radiation, ROS was measured.

$* \mathrm{p}<0.05$ when compared with non-IR cells

$\# \mathrm{p}<0.05$ when compared with IR cells 


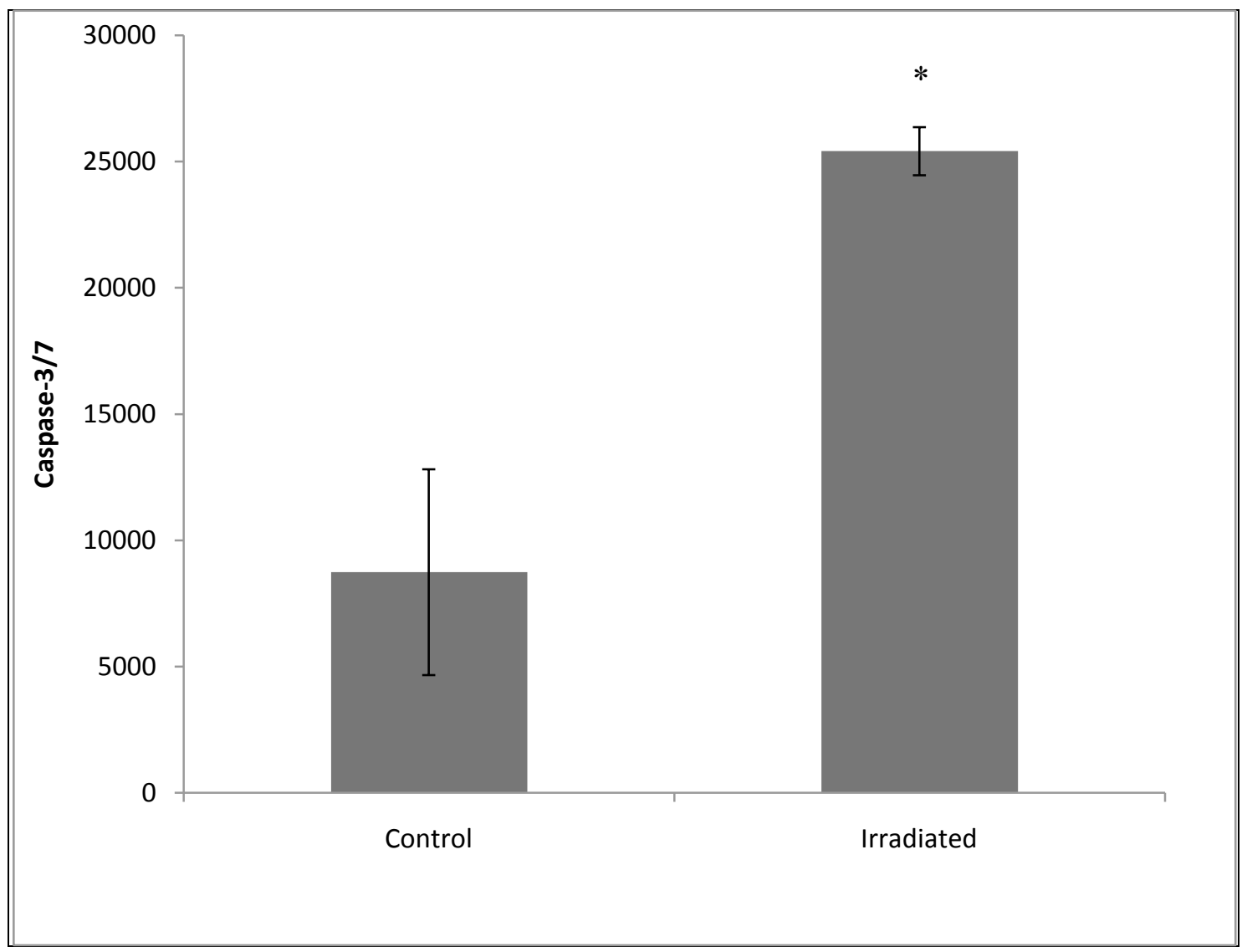

Figure 3-10 Caspase-3/7 Activity 24 Hours post CM Transfer.

U937 cells were irradiated ( 7 Gy) and allowed to incubate for 24 hours. Medium was removed from irradiated cells and placed on unirradiated bystander cells. 24 hours after medium transfer, Caspase-3/7 was measured with luminescent plate reader.

$* \mathrm{p}<0.05$ 


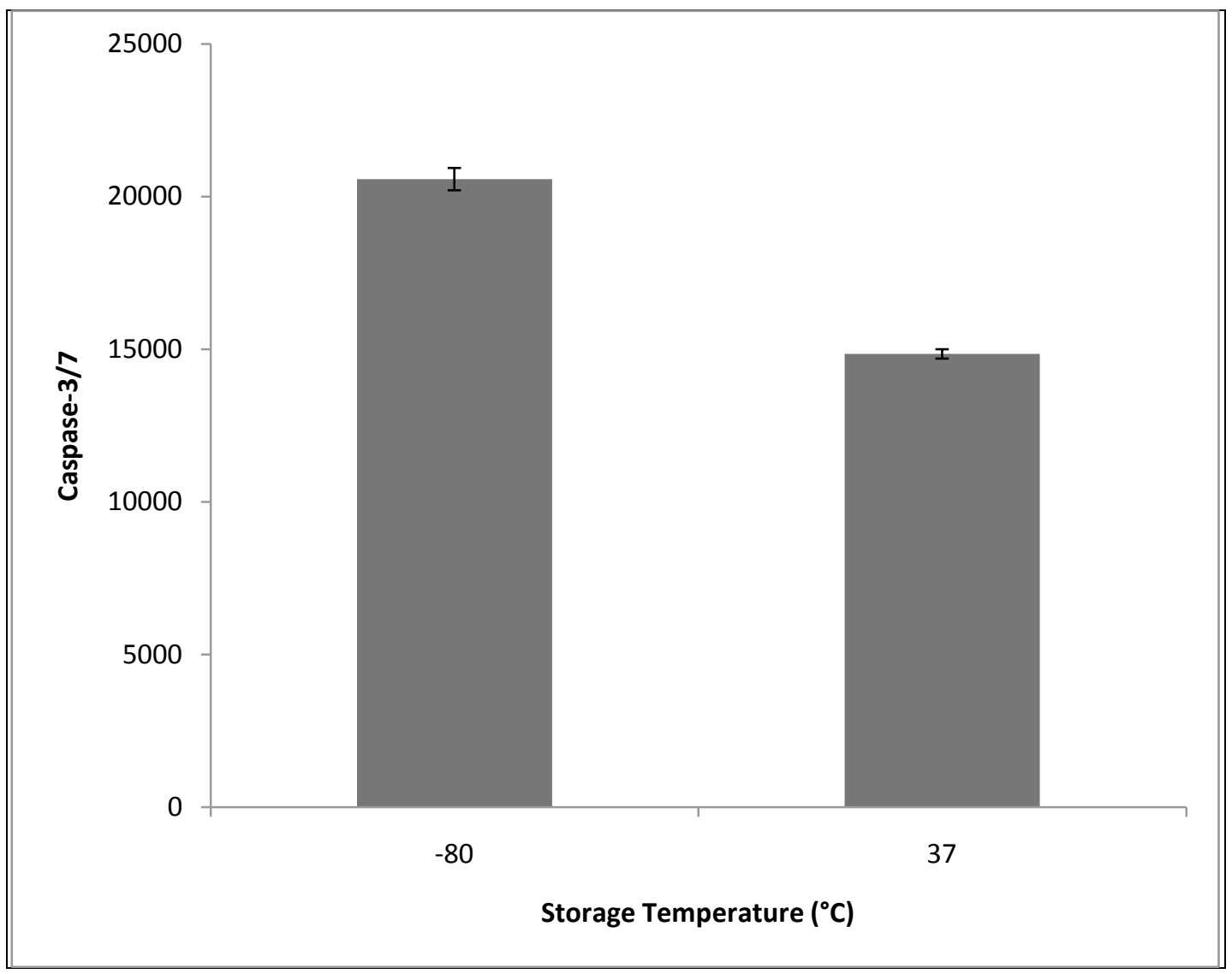

Figure 3-11 Stability of Caspase-3/7 in Cell Culture Medium.

U937 cells were irradiated ( 7 Gy) and allowed to incubate for 24 hours. Medium was removed from irradiated cells and either frozen $\left(-80^{\circ} \mathrm{C}\right)$ or stored at culture conditions $\left(37^{\circ} \mathrm{C}\right)$ for 24 hours. Caspase-3/7 was measured with luminescent plate reader. 


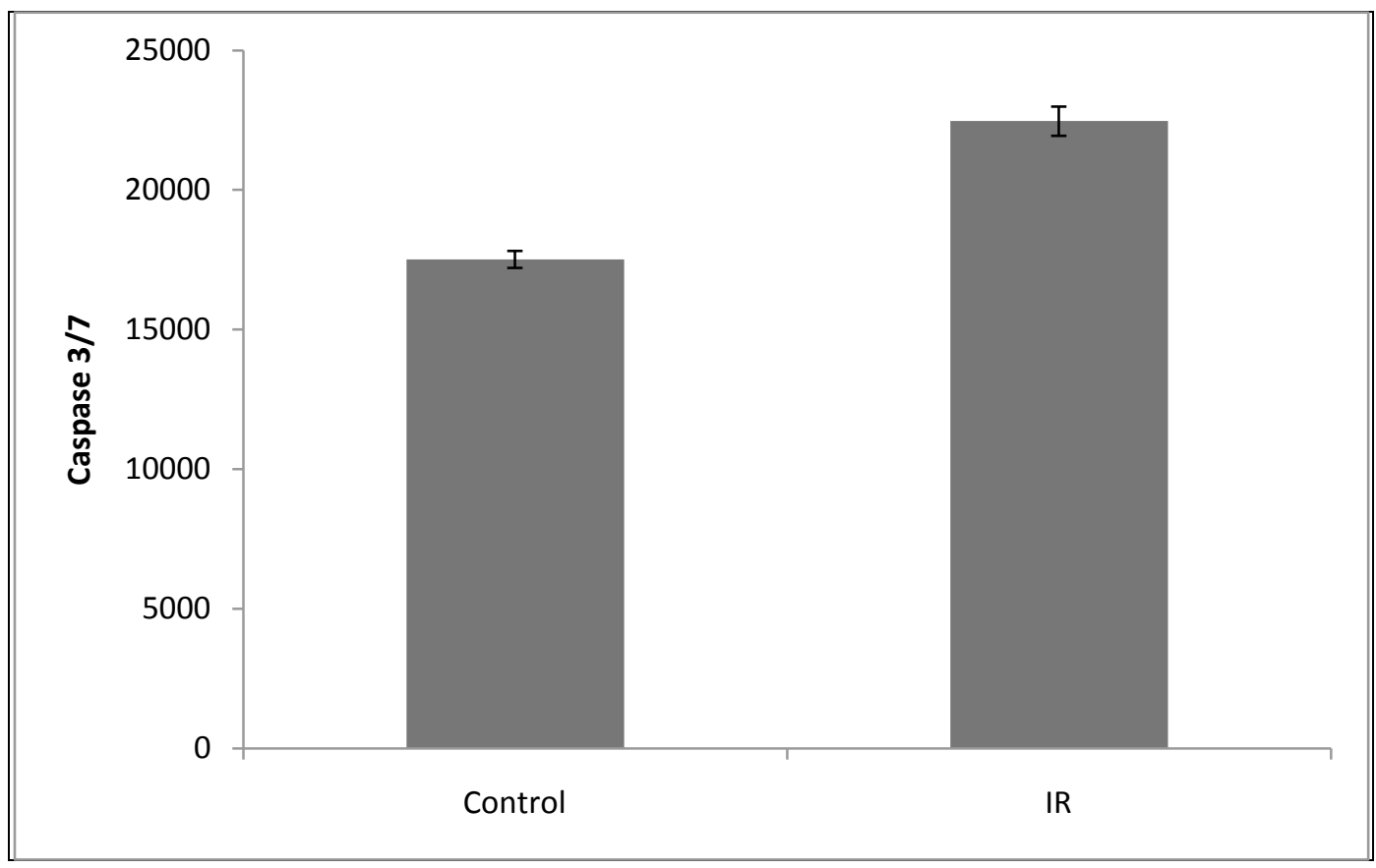

Figure 3-12 Soluble Caspase-3/7 24 Hours post CM Transfer.

U937 cells were irradiated ( 7 Gy) and allowed to incubate for 24 hours. Medium was removed from irradiated cells and transferred to unirradiated bystander cells. Cells and conditioned medium were allowed to incubate for an additional 24 hours. Caspase-3/7 (luminescence) was measured by plate reader in cell-free medium. 


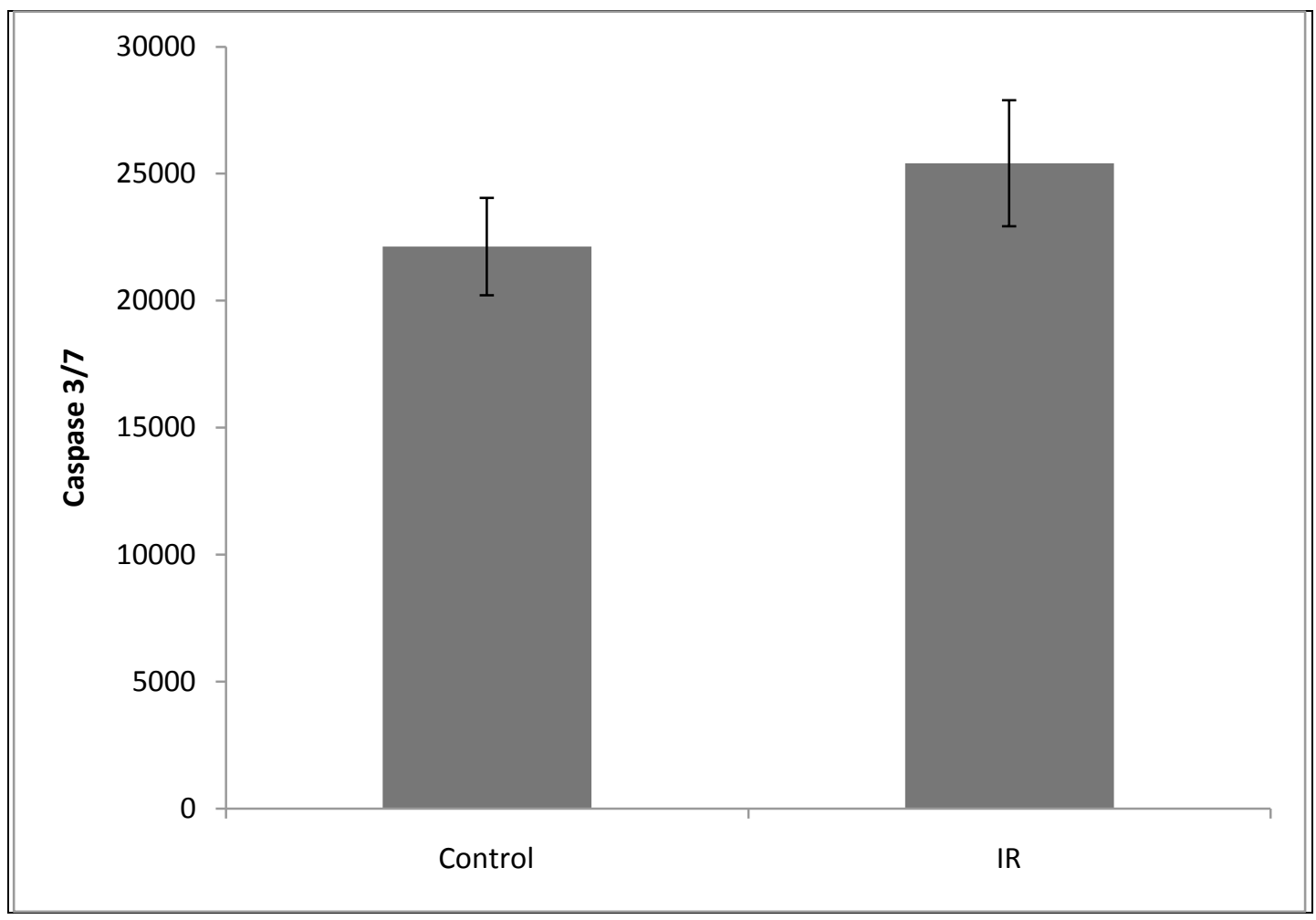

Figure 3-13 Cellular Caspase-3/7 from Bystander Cells 24 Hours post CM

Transfer.

U937 cells were irradiated ( 7 Gy) and allowed to incubate for 24 hours. Medium was removed from irradiated cells and transferred to unirradiated bystander cells with KZ-41 $(0-100 \mu \mathrm{M})$. Cells and conditioned medium was allowed to incubate for an additional 24 hours. Caspase-3/7 (luminescence) was measured by plate reader in washed cells. 


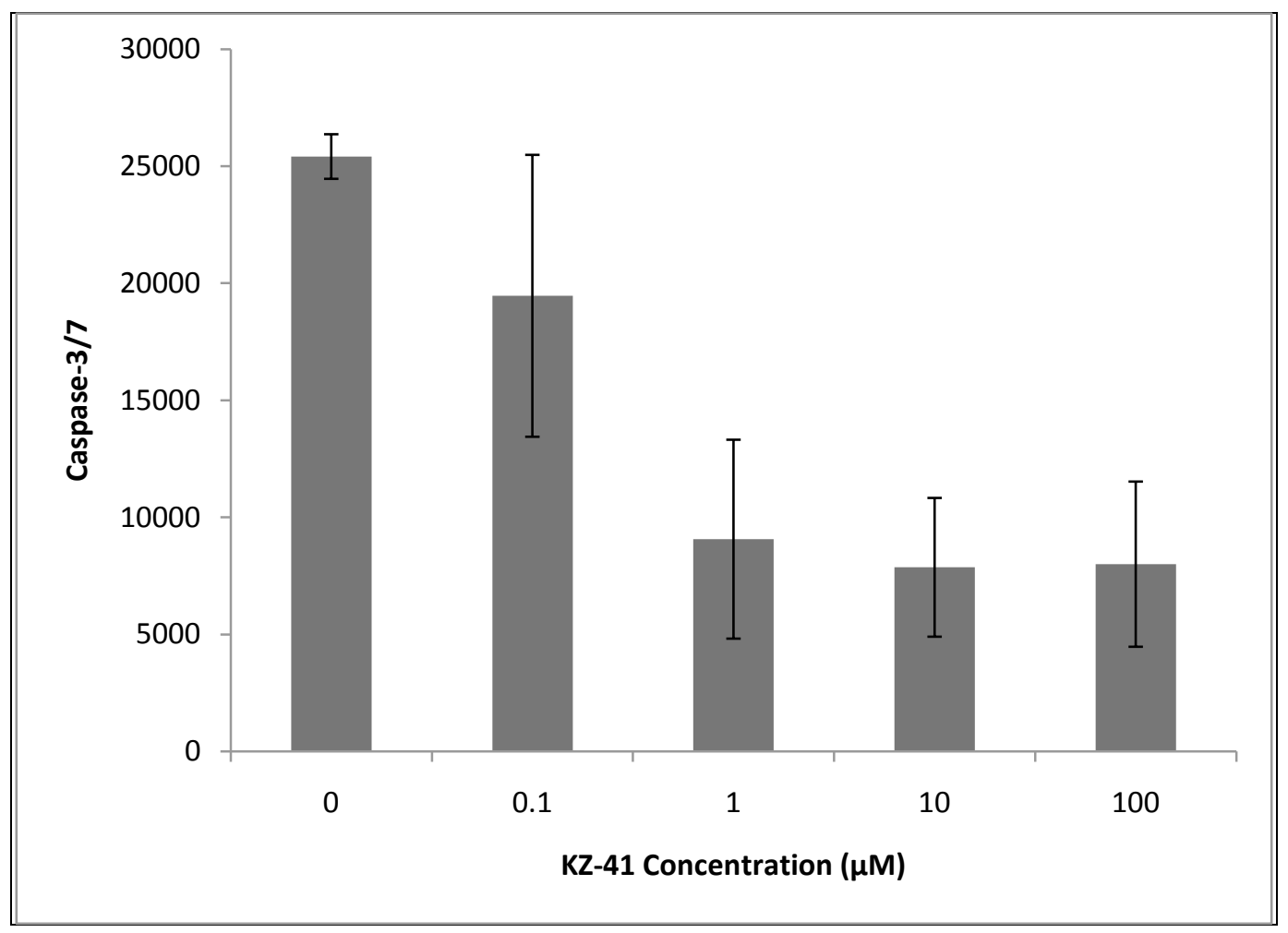

Figure 3-14 Effect of KZ-41 on the Radiation-Induced Bystander Effect. U937 cells were irradiated ( 7 Gy) and allowed to incubate for 24 hours. Medium was removed from irradiated cells and transferred to unirradiated bystander cells with KZ-41 $(0-100 \mu \mathrm{M})$. Cells and conditioned medium was allowed to incubate for an additional 24 hours. Caspase-3/7 (luminescence) was measured by plate reader. 


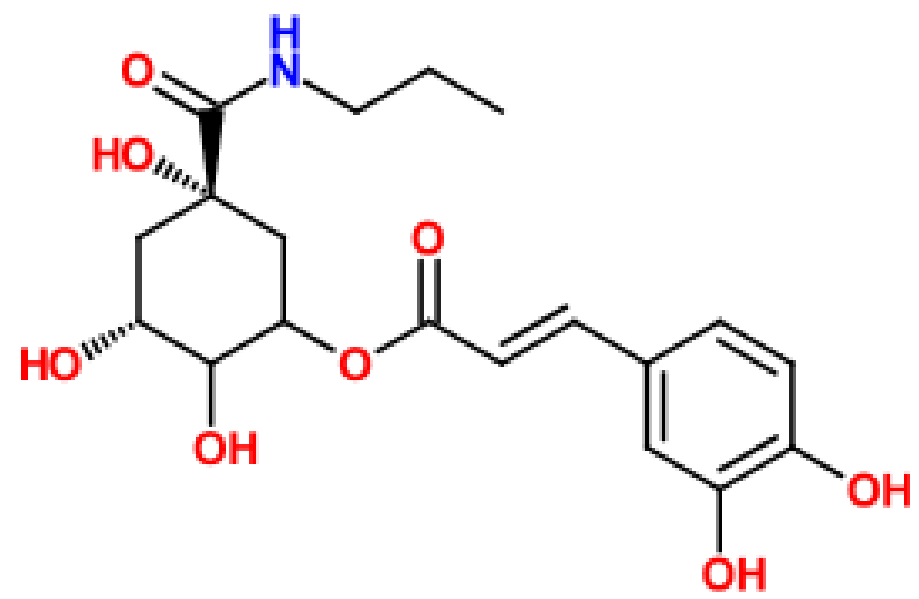

Figure 3-15 Chemical Structure of KZ-157. 


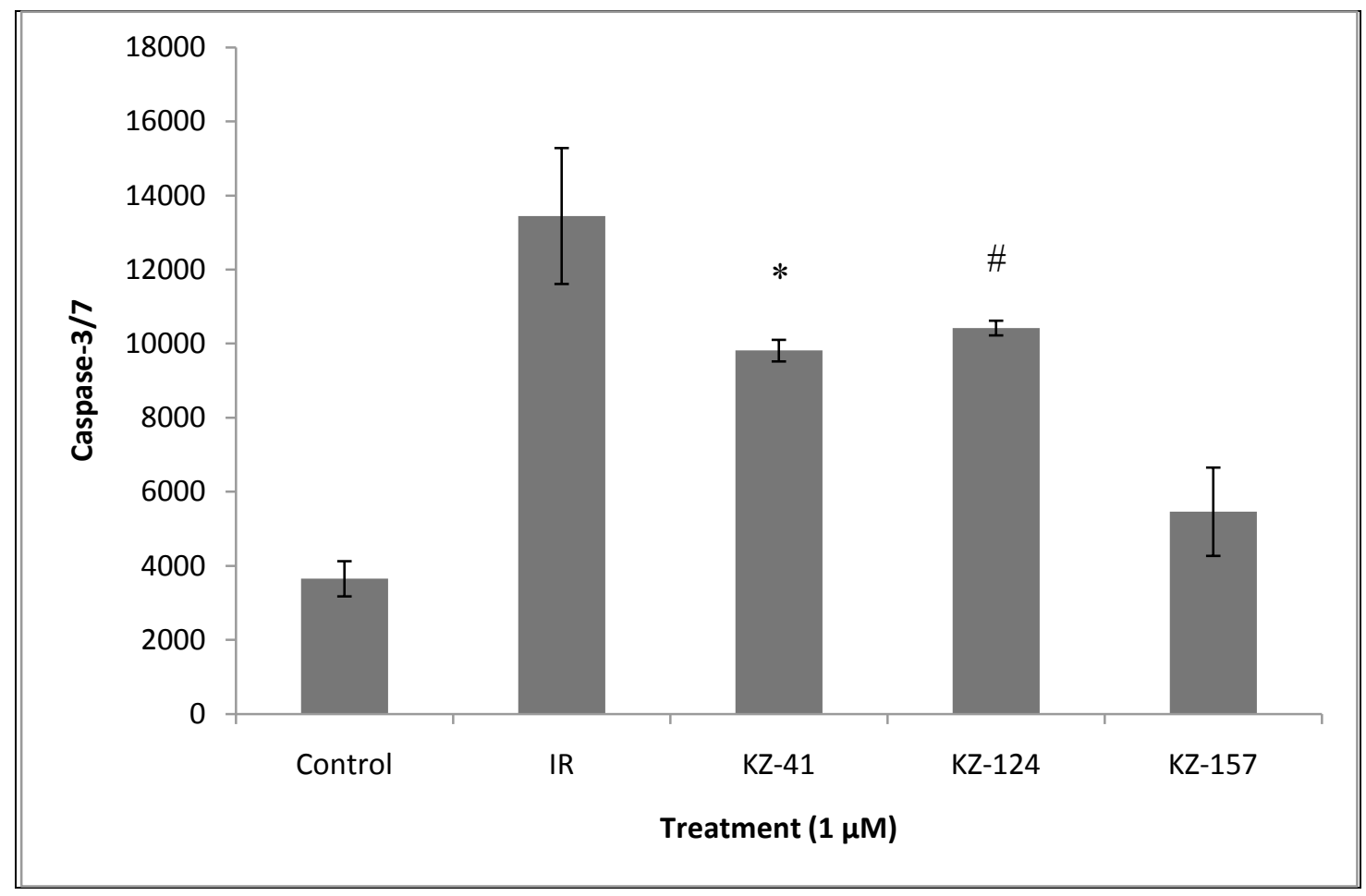

Figure 3-16 Next-Generation Analog Screening.

U937 cells were irradiated ( 7 Gy) and allowed to incubate for 24 hours. Medium was removed from irradiated cells and transferred to unirradiated bystander cells with treatments (KZ-41 and KZ-157 at $1 \mu \mathrm{M})$. Cells and conditioned medium was allowed to incubate for an additional 24 hours. Compounds were screened for their potential to reduce Caspase-3/7 compared to KZ-41.

$* \mathrm{p} \leq 0.05, \# \mathrm{p}<0.01$ when compared to KZ-41 
time-dependent effect in TNF- $\alpha$ concentration, with a peak beginning at 24 hours after IR (Figure 3-7). We also established that although KZ-41 has no effect on Caspase-3 activation when administered prior to IR, there is a dose-dependent reduction in TNF- $\alpha$ 48 hours after IR (Figure 3-8). KZ-41 was also effective in reducing reactive oxygen species levels, another player in the propagation of radiation-induced injury, and possibly applicable in the bystander effect [36]. We demonstrated that a bystander effect can be elicited in the U937 cell line through soluble factors by a series of conditioned medium transfer experiments (Figure 3-10). We hypothesize that a variety of soluble factors such as TNF- $\alpha$, TRAIL, and Fas ligand are the initiators of this bystander effect, and once the bystander cells are exposed to these soluble factors, they initiate the death cascade. We believe that the death cascade progresses through both mitochondrial dependent and independent pathways.

Caspase- $3 / 7$ is not stable at $37^{\circ} \mathrm{C}$, which is the ambient temperature used during the experiments, therefore, only a fraction of the Caspase 3/7 measured at the 24-24 time point is residual from the conditioned medium transfer (Figure 3-11). Also, conditioned medium from irradiated cells may be collected and stored at ${ }^{-} 80^{\circ} \mathrm{C}$ for use in these experiments with success; however, there is some loss of potency in inducing Caspase3/7 in untreated cells (data not shown). KZ-41 dose-dependently reduced Caspase-3/7 levels 24 hours after transfer of conditioned medium taken from cells 24 hours after irradiation (Figure 3-14).

Although we continue to evaluate the efficacy and mechanism of KZ-41 as a radiomitigant, we recognize the importance in discovering a potent next-generation compound for use in Acute Radiation Syndrome. We, therefore, conducted a screen of next generation KZ-41 analogs in the U937 bystander effect model. The caffeic acid ester of KZ-41 (KZ-157) was shown to be significantly more potent at reducing Caspase$3 / 7$ in this bystander effect model, compared to KZ-41 alone (Figure 3-16). Due to published reports on the value of antioxidants for use in radioprotection coupled with our screening data, we will move forward with pre-clinical pharmaceutical characterization of chlorogenic acid analogs, specifically KZ-157 (Figure 3-15). We hypothesize that KZ-157 will have more potent life-saving effect in the in vivo survival studies due to the effect of the antioxidant (caffeic acid) portion of the molecule in addition to the proven benefit of KZ-41. 


\section{CHAPTER 4. PHARMACOKINETIC MODELING OF NEXT GENERATION QUINIC ACID ANALOGS}

\section{INTRODUCTION}

The interest in developing natural products for various indications has rapidly grown in recent years. Inflammation plays a vital role in many disease states, and is therefore a fundamental focus for developing natural products. Central in natural antiinflammatory agents are antioxidants. Radiation injury is one such disease state where antioxidants have shown a proven benefit. The only Food and Drug Administration approved drug for the indication of radioprotection is Amifostine and some of its efficacy may be attributed to free radical scavenging properties [12]. Amifostine, however, does not retain the attractive low toxicity profile of many natural products.

Many antioxidants are naturally occurring in food. One such antioxidant is chlorogenic acid. There are large quantities of chlorogenic acid in coffee, blueberries, chocolate, beer, tea, and many other commonly consumed foods and beverages [39-42]. Chlorogenic acid is the ester of quinic acid and caffeic acid (CA), and may be broken back into its component parts by esterases found in the blood or by enzymatic degradation by gut bacteria $[40,43]$. Once separated, quinic acid has very low bioavailability due to rapid degradation by gut bacteria. As a result of the low bioavailability, the biological activity of chlorogenic acid is attributed to the antioxidant potential of caffeic acid.

Our goal was to retain the active portion of chlorogenic acid (caffeic acid) and to substitute a known biologically active molecule for the quinic acid portion. We substituted KZ-41 for the QA portion of the molecule because of its proven efficacy in our desired setting, Acute Radiation Syndrome. The result was KZ-157 (Figure 4-1), a next generation KZ-41 molecule and chlorogenic acid derivative.

Theoretically, KZ-157 is a pro-drug, however, it is unique in that we hypothesize that its primary metabolites (caffeic acid and KZ-41) would also be active. In order to achieve initial pharmaceutical profiling of KZ-157, an analytical method was required so that KZ-157 and its metabolites could be measured in a biological matrix.

\section{MATERIALS AND METHODS}

\section{Animals}

Double or single cannulated male Sprague-Dawley rats weighing 200-300g obtained from Harlan Laboratories (Indianapolis, IN) were used for this study. The locations for the cannula were the jugular vein and/or the femoral vein. Animals bearing 
single cannula were used for oral dosing studies, and animals bearing double cannula were used for intra venous (I.V.) studies. All animal experiments were submitted,

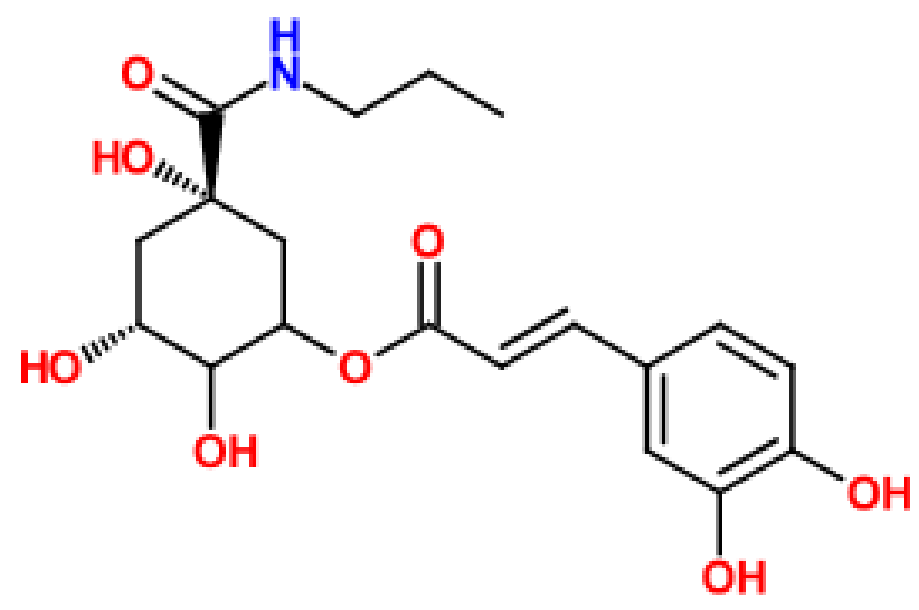

Figure 4-1 Chemical Structure of Chlorogenic Acid Derivative KZ-157. 
reviewed, and approved (Protocol \# 1629) by the University of Tennessee Animal Care and Use Committee.

\section{Treatments}

A weight based dosing strategy was used in these studies. A dose of $1 \mathrm{mg} / \mathrm{kg} \mathrm{KZ}$ 157 (Figure 4-1) was administered to each animal based on the weight of the subject the morning of dosing via the intravenous or oral rout. The dosing solution was $10 \mathrm{mg} / \mathrm{mL}$, therefore a total volume ranging from $0.20-0.26 \mathrm{~mL}$ was delivered to each animal.

\section{PK Sampling Strategy}

The blood sampling strategy was the same for both oral and I.V. studies, and was as follows: $0,5,15,30,45,60,120,240,480,720$, and 1440 minutes after drug administration. Blood was collected from the jugular vein cannula (JVC), and placed in a BD Plasma Separator Microtainer ${ }^{\circledR}$ (BD Biosciences, San Jose, CA) containing lithium heparin, gently mixed, and placed on ice until processing JVC was flushed and the collected blood volume was replaced with $0.9 \%$ Sodium Chloride (NS) following each blood collection.

\section{Pharmacokinetic Data Analysis}

Plasma concentration-time data were analyzed using non-compartmental analysis in WinNonlin 5.0.1 (Pharsight Corporation, Mountain View, CA). The maximum plasma concentration $\left(\mathrm{C}_{\max }\right)$ observed after oral dosing and the time at which it was observed $\left(\mathrm{T}_{\max }\right)$ were determined by direct inspection of the individual plasma concentration-time profiles. The terminal half-life $\left(\mathrm{t}_{1 / 2}\right)$ was calculated as 0.693 divided by the terminal phase rate constant $\left(\lambda_{z}\right)$.

$$
\mathrm{T}_{1 / 2}=\frac{0.693}{\lambda \mathrm{z}}
$$

The area under the plasma concentration-time curve from time 0 to infinity $\left(\mathrm{AUC}_{0-\infty}\right)$ was calculated by the trapezoidal rule with extrapolation to time infinity. The total plasma clearance (CL) was calculated as the dose (D (ng)) divided by the observed $\mathrm{AUC}_{0-\infty}$.

$$
\mathrm{CL}=\frac{\mathrm{D}}{\mathrm{AUC}_{0-\infty}}
$$


The fraction of drug absorbed (F) was calculated by dividing the oral $\mathrm{AUC}_{0-\infty}$ by the intravenous $\mathrm{AUC}_{0-\infty}$. The oral clearance $\left(\mathrm{CL}_{\text {oral }}\right)$ was obtained by dividing the $\mathrm{CL}$ by F.

$$
\mathrm{F}=\frac{\mathrm{AUC}_{0-\infty, \mathrm{I} . \mathrm{V} .}}{\mathrm{AUC}_{0-\infty, \text { oral }}} \quad \mathrm{CL}_{\text {oral }}=\frac{\mathrm{CL}}{\mathrm{F}}
$$

Volume of distribution (V) was calculated as the dose divided by the product of $\mathrm{AUC}_{0-\infty} \lambda_{\mathrm{z}}$. The volume of distribution following the oral dose $\left(\mathrm{V}_{\text {ora }} \mathrm{l}\right)$ was determined by dividing the volume by $\mathrm{F}$.

$$
\mathrm{V}=\frac{\mathrm{D}}{\mathrm{AUC}_{0-\infty} * \lambda \mathrm{z}} \quad \mathrm{V}_{\text {oral }}=\frac{\mathrm{V}}{\mathrm{F}}
$$

\section{LC-MS/MS Method}

Chromatographic separation was carried out using Shimadzu (Columbia, MD) LC-10ADvp pumps with a Leap (Carrboro, NC) CTC PAL autosampler HPLC system. The analytes were separated with a methanol-2mmol/L ammonium acetate solution $(65 / 35, \mathrm{v} / \mathrm{v})$ mobile phase and an Agilent Zorbax C18 column $(5 \mu \mathrm{m}$ particle size, $4.6 \mathrm{~mm}$ $\mathrm{x} 150 \mathrm{~mm}$ ). A flow rate of $0.5 \mathrm{~mL} / \mathrm{min}$ and an injection volume of $10 \mu \mathrm{L}$ were used. The total LC run time was 8 minutes. The column and autosampler were maintained at ambient temperature for the duration of the method. Detection of the analytes and internal standard was performed on an API 4000 Q TRAP mass spectrometer equipped with an electrospray ion source (Applied Biosystems Sciex, Foster City, CA) operated in negative-ion mode. The LC and MS parameters were controlled by Analyst software version 5.0. Multiple reaction monitoring (MRM) scan type was used to monitor the transition from precursor to product ion for KZ-157 $\mathrm{m} / \mathrm{z} 394 \rightarrow 161$, CA $\mathrm{m} / \mathrm{z} 179 \rightarrow 135$, KZ-41 $m / z 232 \rightarrow 85$, and IS $m / z 352 \rightarrow 191$. The dwell time for all analytes was 250 msec.

\section{Standard Samples}

Stock solutions of CA, KZ-41, and KZ-157 were prepared in methanol at a concentration of $1.0 \mathrm{mg} / \mathrm{mL}$. The stock solutions were combined and further diluted in methanol to make working solutions for standards $(20000,10000,5000,2500,1000,500$, and $100 \mathrm{ng} / \mathrm{mL}$ ). The IS stock solution was further diluted in methanol to make an IS working solution $(1500 \mathrm{ng} / \mathrm{mL})$. Analyte working solutions were then spiked (10X) into rat plasma to make calibration standards. The resulting concentrations of standards and 
QC samples were 2000, 1000, 500, 250, 100, 50, 25, and $12.5 \mathrm{ng} / \mathrm{mL}$. Calibration standards were prepared fresh daily.

\section{Sample Extraction from Plasma}

Sample extraction was optimized based on a method previously published [44]. Plasma aliquots were processed 8 at a time to ensure minimum time between thaw and processing. Aliquots of $100 \mu \mathrm{L}$ plasma were thawed on ice, and immediately mixed with $10 \mu \mathrm{L}$ IS, and $1 \mathrm{~mol} / \mathrm{L}$ hydrochloric acid and vortexed for one minute. Ethyl-ether-ethyl acetate $(1: 1, \mathrm{v} / \mathrm{v})$ was added to the sample and vortexed for 10 minutes. The sample was then centrifuged at $10,000 \mathrm{x}$ g for 10 minutes at $4^{\circ} \mathrm{C}$. The supernatant was transferred to a clean tube and evaporated to dryness at $35^{\circ} \mathrm{C}$ under a stream of nitrogen $(5 \mathrm{psi})$. The residue was dissolved in $25 \mu \mathrm{L}$ methanol, vortexed for 1 minute and centrifuged for 1 minute at $10,000 \mathrm{x}$. The supernatant was transferred into an autosampler vial where 10 $\mu \mathrm{L}$ was injected into the LC-MS/MS system for analysis.

\section{Rat Microsomal Stability}

The role of Phase I metabolism was investigated using pooled rat liver microsomes (Cellzdirect, Austin, TX) by monitoring disappearance of parent compound. $25 \mu \mathrm{L}$ Compound (50 $\mu \mathrm{M}$ in 0.1 phosphate buffer) was combined with $25 \mu \mathrm{L}(10 \mathrm{mg} / \mathrm{mL})$ microsomal protein solution and NADPH regenerating solution $\left(1.3 \mathrm{mM} \mathrm{NADP}^{+}, 3.3\right.$ $\mathrm{mM}$ glucose-6-phosphate, $3.3 \mathrm{mM} \mathrm{MgCl}_{2}$, and 1 unit/mL glucose-6-phosphate dehydrogenase in $\mathrm{pH} 7.4$ phosphate buffer solution). Reactions were incubated at $37^{\circ} \mathrm{C}$ for $0,15,30,45,60$, and 90 minutes. Duplication of this experiment was performed simultaneously with the following modifications: (1) heat inactivated (denatured) microsomal protein was used as a negative control and (2) a second known substrate (propanalol) was used as a positive control for microsomal activity. At the end of incubation, $10 \mu \mathrm{L}$ internal standard (KZ-124 $1 \mu \mathrm{g} / \mathrm{mL}$ in Methanol) and $10 \mu \mathrm{L}$ Hydrochloric Acid (1M) was added and mixed. $0.8 \mathrm{~mL}$ ethyl-ether-ethyl acetate was added and the mixture was vortexed on high for 3 minutes. Samples were centrifuged at $10,000 \mathrm{rpm}$ at $4^{\circ} \mathrm{C}$ for 10 minutes. The supernatant was divided and stored at ${ }^{-} 80^{\circ} \mathrm{C}$. Prior to analysis, samples were thawed and evaporated to dryness under a stream of nitrogen gas. The pellet was resuspended in $25 \mu \mathrm{L}$ methanol and analyzed by LC$\mathrm{MS} / \mathrm{MS}$.

\section{In Vitro Protein Binding}

Protein binding was analyzed using equilibrium dialysis. RED $^{\circledR}$ (rapid equilibrium dialysis) inserts (Pierce Biotechnology Inc, Rockford, IL) coupled with a base plate was used for this analysis. Concentrations ranging from 0.01 to $10 \mu \mathrm{g} / \mathrm{mL}$ were tested in rat plasma. Plasma was mixed $(200 \mu \mathrm{L})$ with the test compound was placed in one side of the chamber and $350 \mu \mathrm{L}$ blank isotonic phosphate buffer, $\mathrm{pH} 7.4$ was 
placed in the other side of the chamber. The chambers were covered and incubated with $100 \mathrm{rpm}$ orbital shaking at $37^{\circ} \mathrm{C}$ for $4-6$ hours to allow equilibrium to be achieved.

Volume of each chamber was checked at the end of the incubation to ensure no volume shift occurred. $50 \mu \mathrm{L}$ aliquots of plasma and buffer were separately analyzed to determine the drug concentration using LC-MS/MS. The percent free compound was calculated as the ratio of the compound concentration in the buffer and plasma.

\section{RESULTS}

\section{LC-MS/MS Method}

Each analyte monitored had a distinct retention time under the established conditions. The retention times for Caffeic Acid, KZ-41, KZ-157 and the internal standard were 2.38, 3.27, 3.9, and 4.44 minutes respectively (Figures 4-2, 4-3, 4-4, 4-5). The retention times were consistent when measured simultaneously (Figure 4-6).

\section{Pharmacokinetics of KZ-157}

KZ-157 demonstrates two-compartment pharmacokinetics (Figure 4-7). Plasma concentrations were below the limit of quantification by 2 hours after both intravenous and oral administration (Figures 4-7, 4-8). KZ-157 appeared rapidly in the plasma after oral administration with the Cmax being less than 15 minutes in all animals. The pharmacokinetic parameters of each animal dosed via the oral and intravenous routes are described in Tables 4-1 and 4-2, respectively. The volume of distribution was 7066 and $1909 \mathrm{~mL}$ for oral and I.V. respectively. The half life was very short at 53 minutes for oral and 34 minutes for I.V. There was a large amount of variability, particularly with the oral dosing regimen. The fraction of drug absorbed following oral dosing is low at approximately $28 \%$ (Table $\mathbf{4 - 3}$ ).

\section{Protein Binding of KZ-157}

The extent of protein binding of KZ-157 to proteins in rat plasma using equilibrium dialysis was determined to be $43.8 \%$ bound.

\section{Microsomal Stability}

KZ-157 is very resistant to metabolism by liver microsomes. Greater than $70 \%$ of KZ-157 remains after a 90 minute incubation with liver microsomes. 


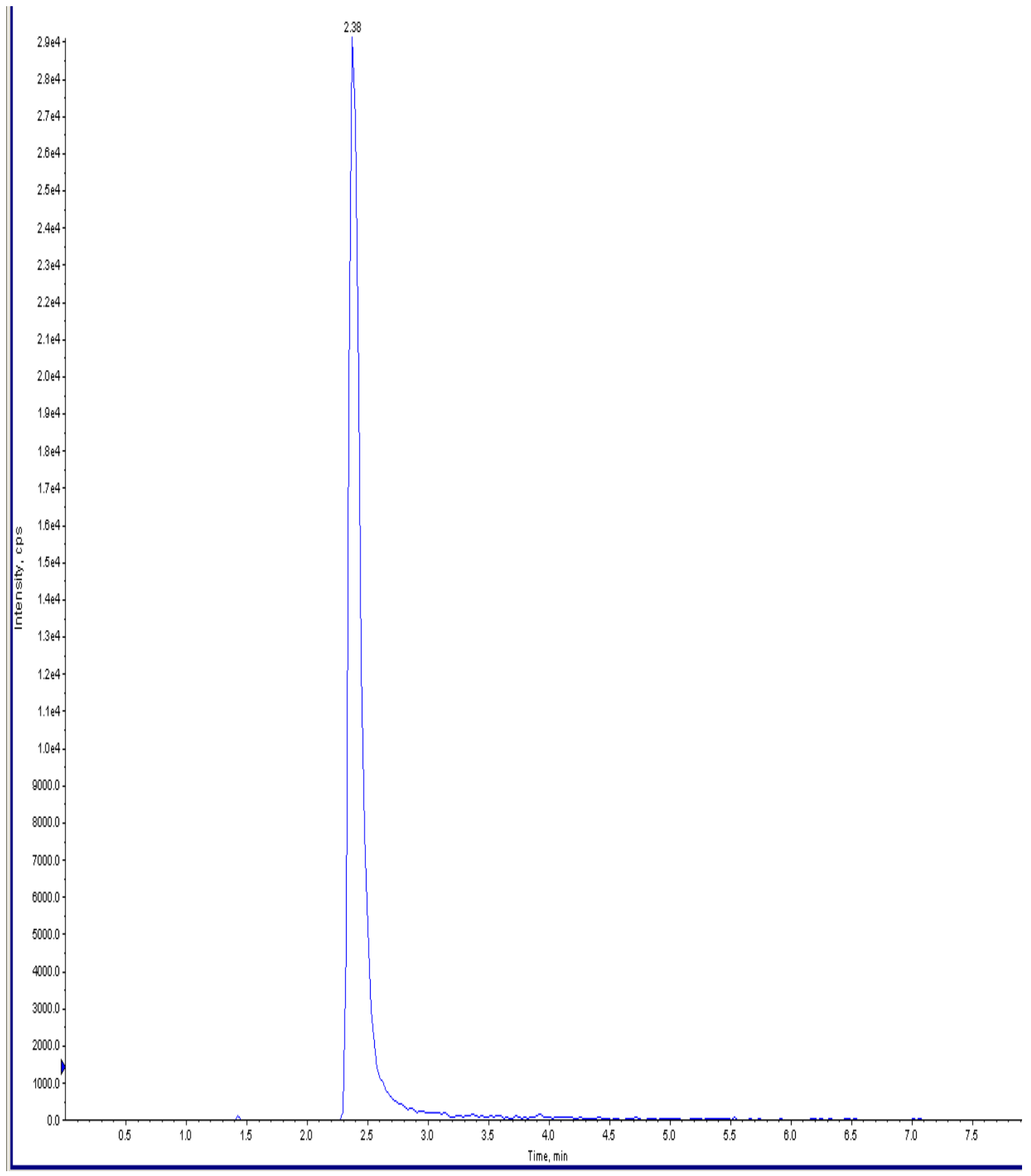

Figure 4-2 Retention Time of Caffeic Acid.

The retention time of caffeic acid, a primary metabolite of KZ-157, was determined under the specified LC-MS/MS conditions as 2.3 minutes. 


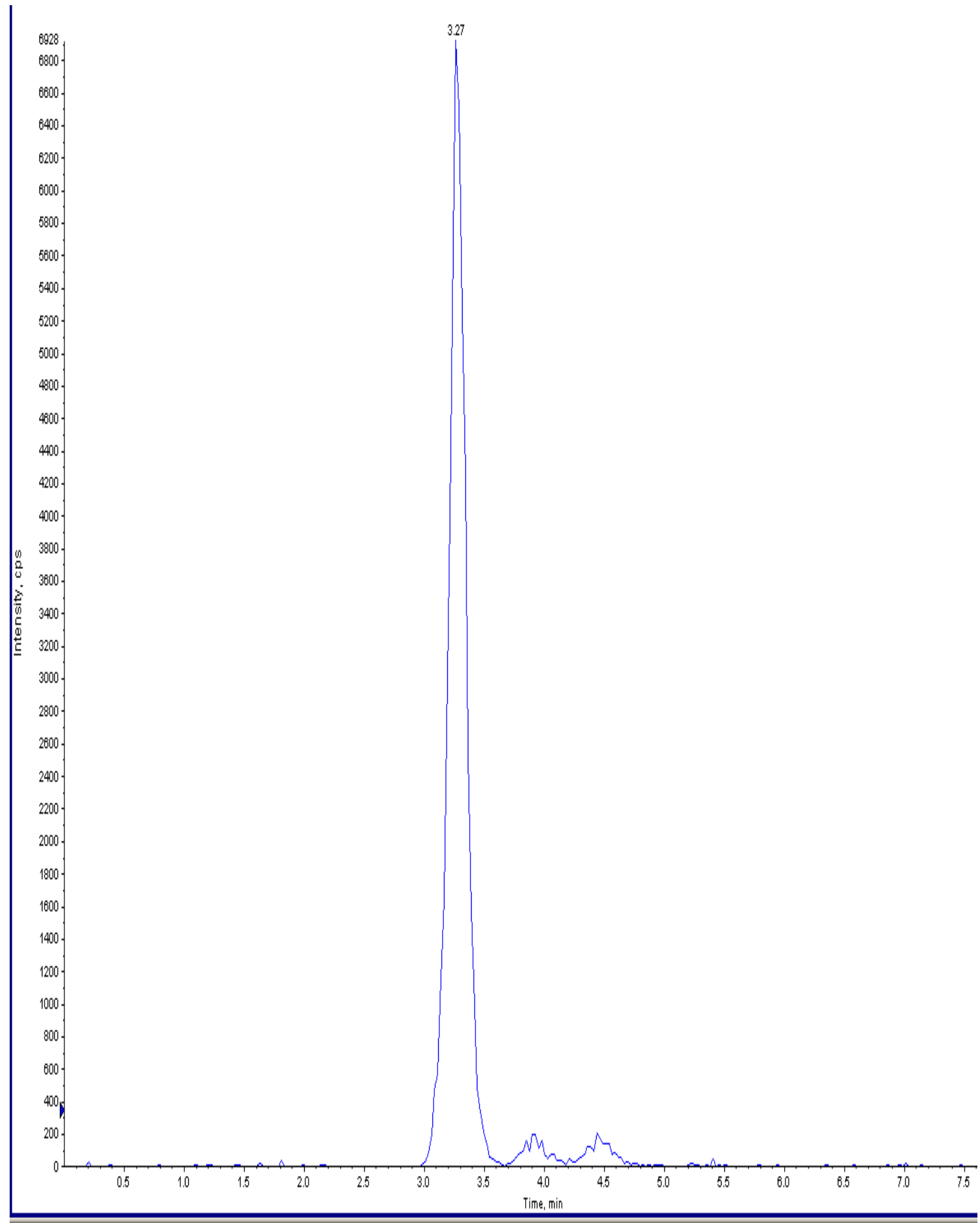

Figure 4-3 Retention Time of KZ-41.

The retention time of KZ-41, a primary metabolite of KZ-157, was determined under the specified LC-MS/MS conditions as 3.27 minutes. 


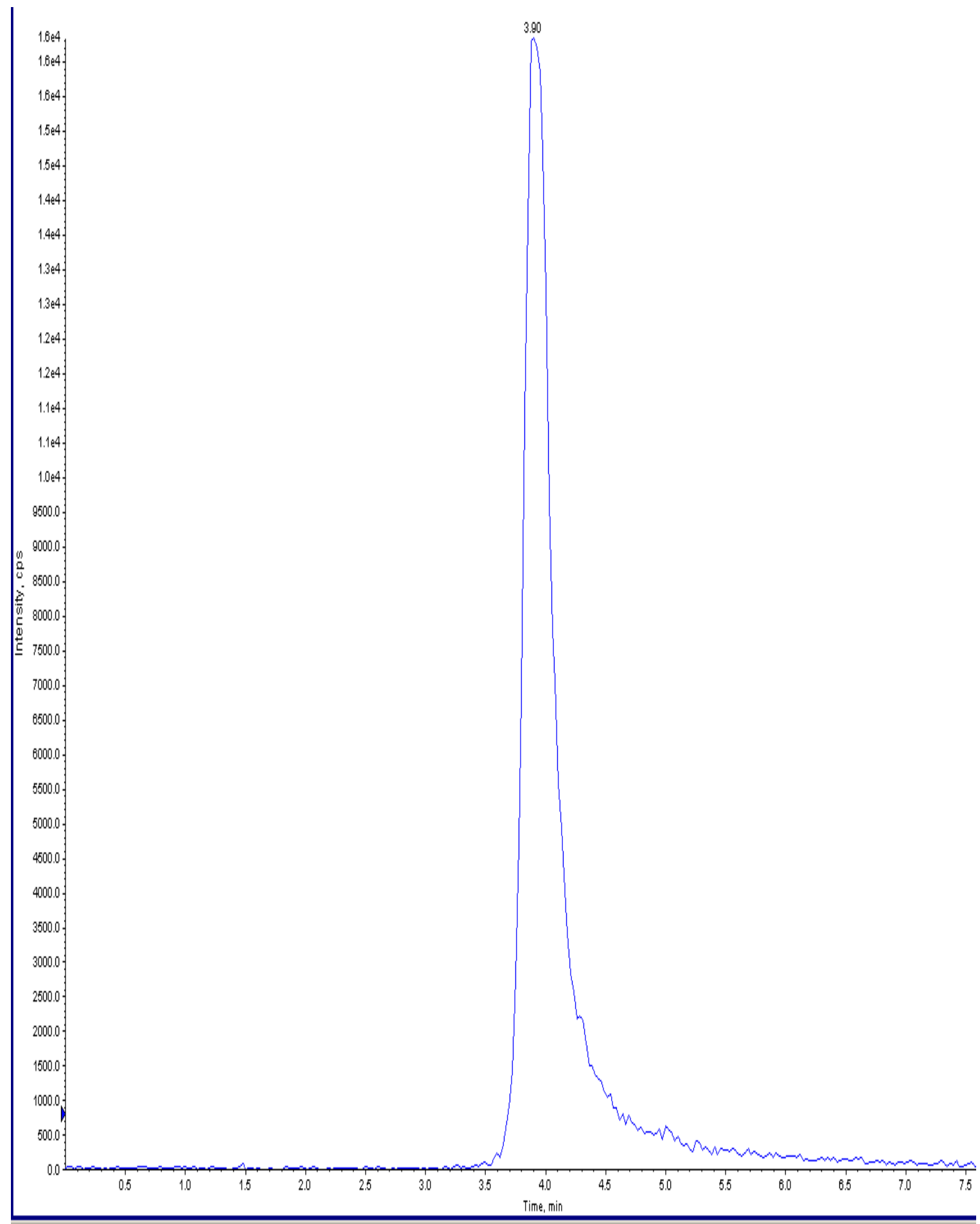

Figure 4-4 Retention Time of KZ-157.

The retention time of KZ-157 was determined under the specified LC-MS/MS conditions as 3.9 minutes. 


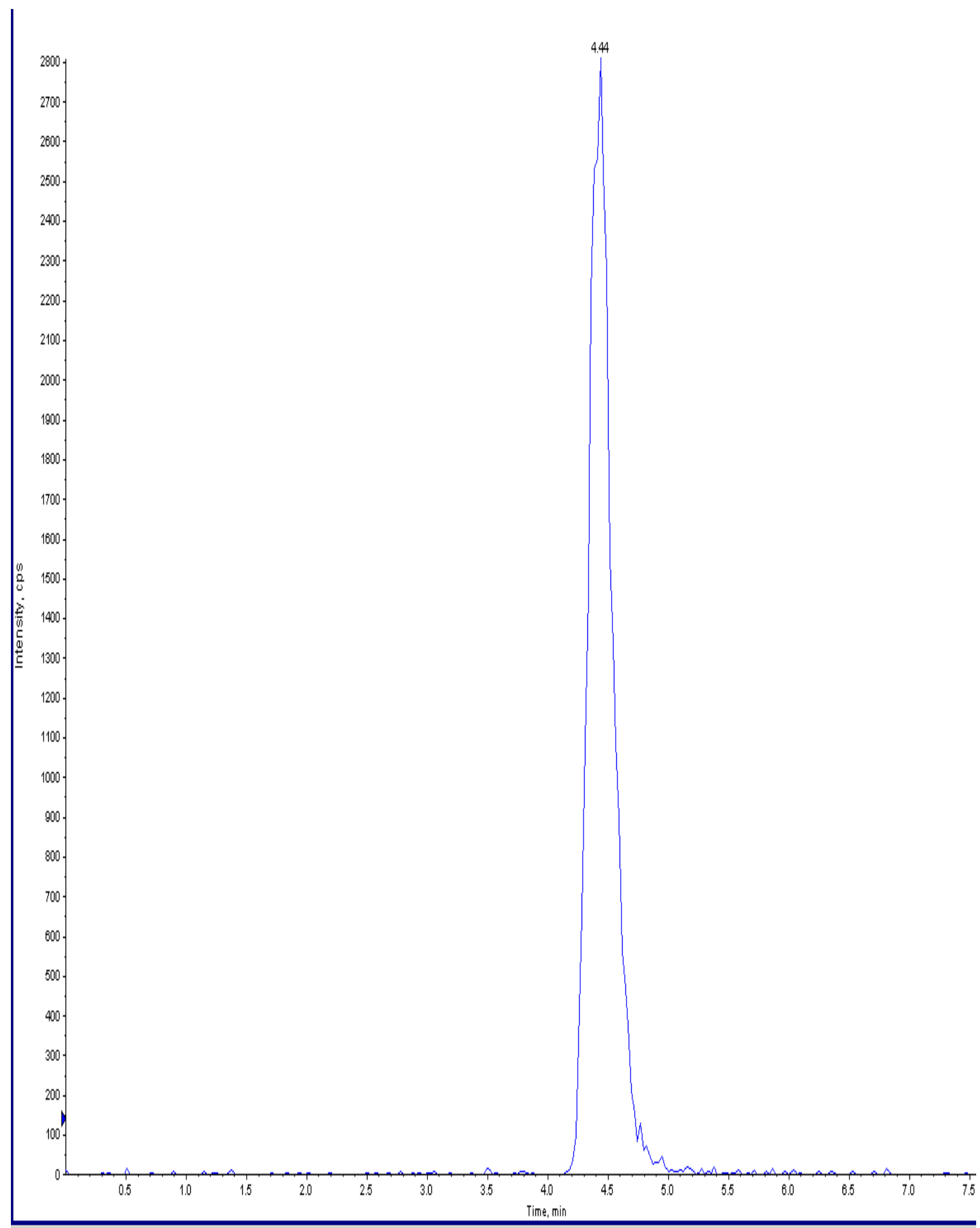

Figure 4-5 Retention Time of IS.

The retention time of the internal standard used in quantification of KZ-157 was determined under the specified LC-MS/MS conditions as 4.44 minutes. 


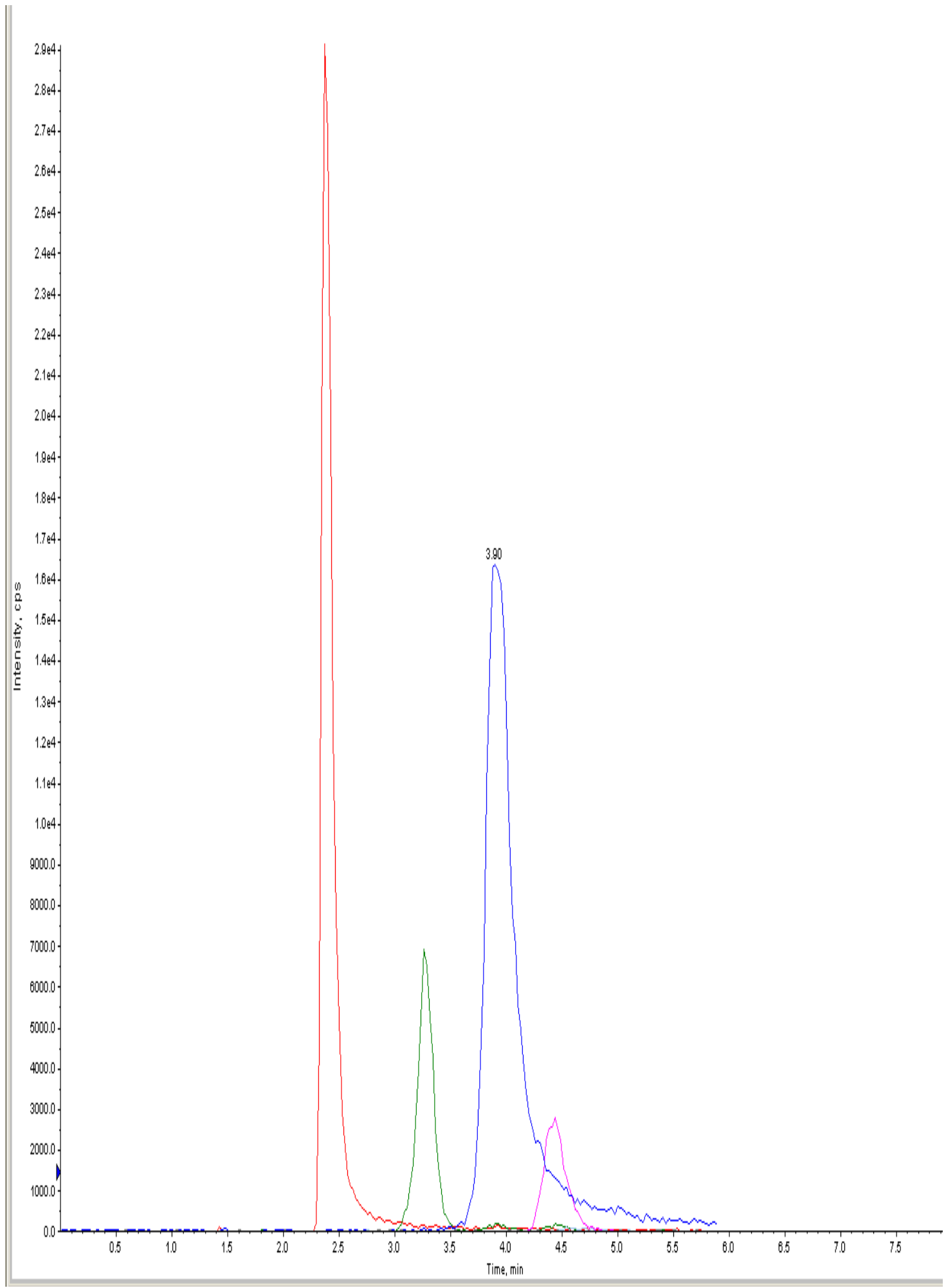

Figure 4-6 Simultaneous Detection of KZ-157 and Primary Metabolites. 


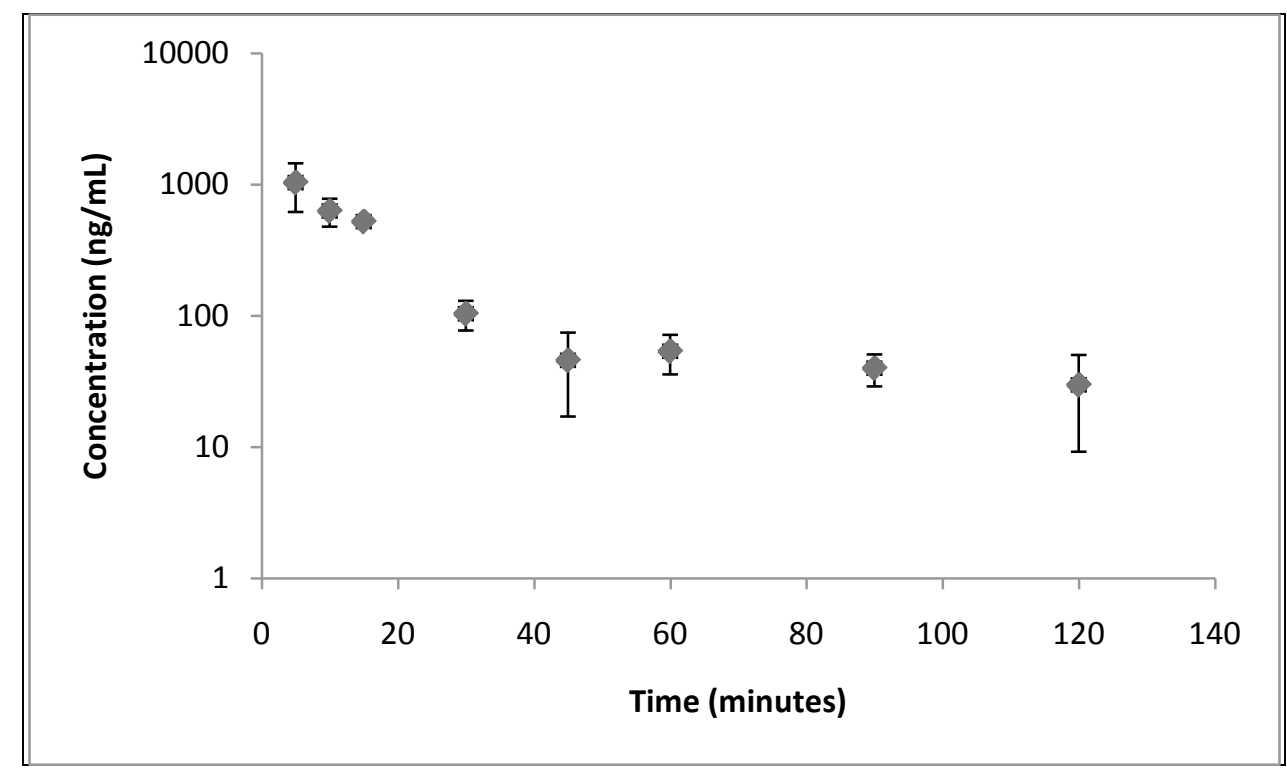

Figure 4-7 Concentration-Time Profile of KZ-157 after Intravenous Administration.

KZ-157 (1mg/kg) was administered intravenously to Sprague-dawley rats $(n=4)$. Serial plasma samples were collected and quantified using LC-MS/MS.

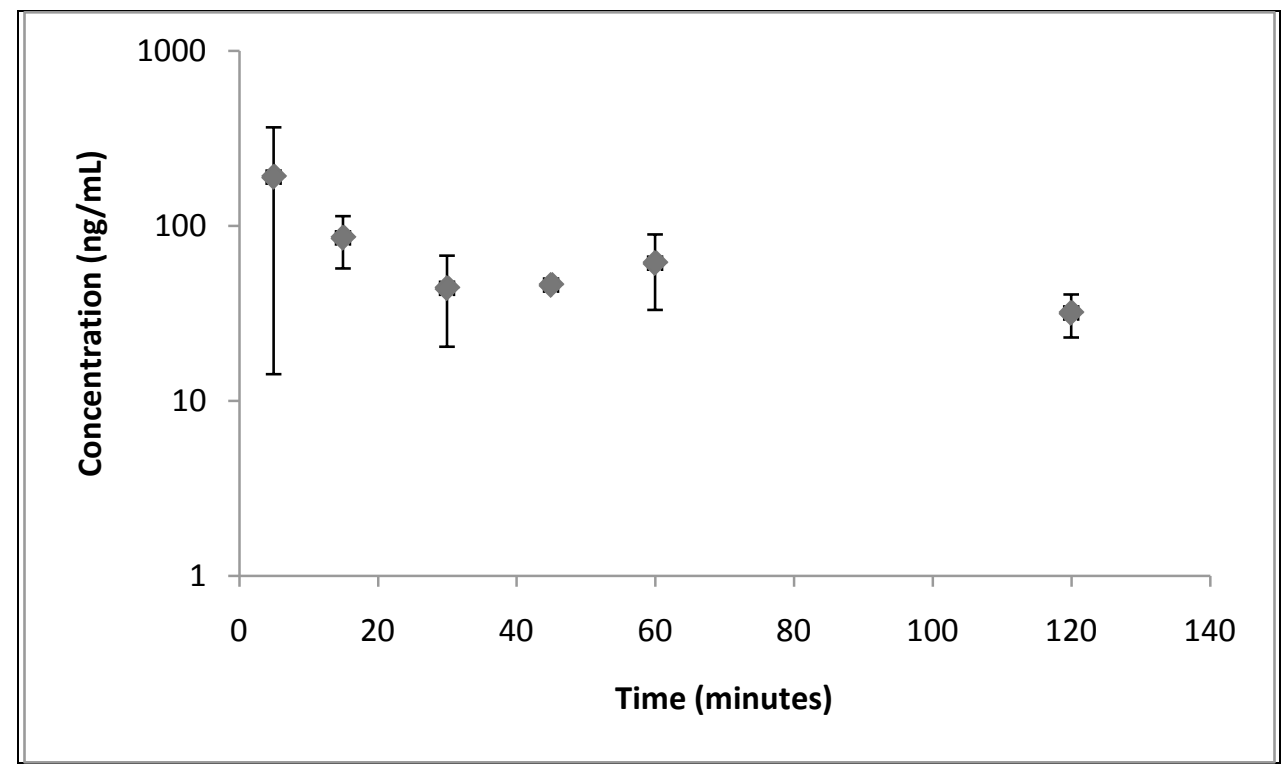

Figure 4-8 Concentration-Time Profile of KZ-157 after Oral Administration. KZ-157 $(1 \mathrm{mg} / \mathrm{kg})$ was administered orally to Sprague-dawley rats $(\mathrm{n}=4)$. Serial plasma samples were collected and quantified using LC-MS/MS. 
Table 4-1 Pharmacokinetic Data for KZ-157 after Oral Administration.

\begin{tabular}{llllll}
\hline Subject & $\begin{array}{l}\mathrm{CL}_{\text {oral }} \\
(\mathrm{mL} / \mathrm{min})\end{array}$ & $\begin{array}{l}\mathrm{Vss}_{\text {oral }} \\
(\mathrm{mL})\end{array}$ & $\begin{array}{l}\text { AUC } \\
(\mathrm{min} * \mathrm{ng} / \mathrm{mL})\end{array}$ & $\begin{array}{l}\mathrm{T}_{1 / 2} \\
(\mathrm{~min})\end{array}$ & $\mathrm{F}$ \\
\hline 5 & 80 & 7570 & 1248 & 65.37736 & \\
6 & 159 & 13636 & 6306 & 59.74138 & \\
7 & 81 & 6773 & 12327 & 57.75 & \\
8 & 286 & 286 & 3491 & 27.94355 & \\
& & & & & 0.28 \\
Mean & 152 & 7066 & 5843 & 53 & \\
Standard Deviation & 97 & 5461 & 4792 & 17 & \\
\hline
\end{tabular}

Table 4-2 Pharmacokintic Data for KZ-157 after Intravenous Administration.

\begin{tabular}{lllll}
\hline Subject & $\begin{array}{l}\text { CL } \\
(\mathrm{mL} / \mathrm{min})\end{array}$ & $\begin{array}{l}\text { Vss } \\
(\mathrm{mL})\end{array}$ & $\begin{array}{l}\text { AUC } \\
(\mathrm{min} * \mathrm{ng} / \mathrm{mL})\end{array}$ & $\begin{array}{l}\mathrm{T}_{1 / 2} \\
(\mathrm{~min})\end{array}$ \\
\hline 1 & 49 & 1733 & 20339 & 25.29197 \\
2 & 50 & 2910 & 20127 & 64.76636 \\
3 & 55 & 1763 & 18209 & 23.97924 \\
4 & 42 & 1231 & 23639 & 22.42718 \\
& & & & \\
Mean & 49 & 1909 & 20579 & 34 \\
Standard Deviation & 5 & 710 & 2254 & 20 \\
\hline
\end{tabular}

Table 4-3 Mean Pharmacokinetic Parameters of KZ-157 after Oral and IV Administration.

\begin{tabular}{lllll}
\hline Route & $\begin{array}{l}\mathrm{T}_{1 / 2} \\
\mathrm{~min}\end{array}$ & $\begin{array}{l}\mathrm{CL} \\
\mathrm{mL} / \mathrm{min}\end{array}$ & $\begin{array}{l}\mathrm{V}_{\mathrm{ss}} \\
\mathrm{mL}\end{array}$ & $\mathrm{F}$ \\
\hline IV & 34 & 49 & 1909 & \\
Oral & 53 & 152 & 7066 & 0.28 \\
\hline
\end{tabular}




\section{DISCUSSION}

KZ-157 is a chlorogenic acid derivative and a next-generation analog of KZ-41. It was shown to potentially be more potent than its precursor KZ-41 in the in vitro bystander effect screen (Figure 3-17). Our aim was to establish initial pharmaceutical profiling of KZ-157. In order to measure this novel chlorogenic acid derivative, we developed an LC-MS/MS method coupled with an extraction method to quantify KZ-157 and its metabolites in a small biological sample.

Other investigators have reported rapid initial absorption and bimodal peaks following a two-compartment model after oral administration of chlorogenic acid [45, 46]. These groups administered high doses (greater than $200 \mathrm{mg} / \mathrm{kg}$ ) and were able to monitor the primary chlorogenic acid metabolites quinic acid and caffeic acid. Our initial aim was to quantify the KZ-157 metabolites CA and KZ-41 and we acknowledge the importance of monitoring them. The dose we administered to the animals was comparatively much lower $(1 \mathrm{mg} / \mathrm{kg})$ than those investigators who conducted metabolite monitoring, and we feel that with a modest increase in the administered dose we will have quantifiable levels of the most common metabolites. There are also conflicting reports of stability in plasma with some suggesting that it is susceptible to esterase activity in rat plasma while stable in human plasma. Our data suggests that KZ-157 is susceptible to plasma or whole blood esterase activity in that we can detect CA and KZ41 analyte peaks as early as 5 minutes after intravenous administration of KZ-157 (data not shown). Modeling these metabolites is critical due to our hypothesis that the parent compound, KZ-157, along with each of the individual metabolites, CA and KZ-41, will have biological activity. It has been reported that chlorogenic acid is poorly absorbed from the intestine, and that bioavailability is largely dependent on individual gut microflora $[40,47]$. Our data suggests that KZ-157 is rapidly absorbed in its native form as indicated by its detection 5 minutes following oral administration.

KZ-157 demonstrates two-compartment pharmacokinetics, and rapid absorption following oral administration. The relative resistance to metabolism by liver microsomes coupled with the rapid elimination observed suggests high susceptibility to other mechanisms of degradation such as esterase activity. The high variability observed during the early time points following oral administration and relatively low bioavailability (as demonstrated by a low fraction absorbed following oral administration) could be partly due to the mechanism by which this compound is absorbed. 


\section{CHAPTER 5. SUMMARY}

There are currently no FDA approved radiomitigators for the treatment of Acute Radiation Syndrome. An urgent need exists for safe and effective drugs that can be administered at least 24 hours after high dose total body irradiation to large numbers of patients. Effects to the hematopoietic system play a central role in the pathogenesis and primary causes of morbidity and mortality in patients presenting with ARS. The hallmark of HEM-ARS is hematopoietic dysfunction. The hematopoietic failure rapidly translates to pancytopenia resulting in a decreased ability to fight infection and increased bleeding potential. Death from HEM-ARS is primarily related to sepsis and hemorrhage. Hematopoietic effects are present in all patients, regardless of the total absorbed dose, in patients affected by ARS due to the presence of overlapping syndromes. In light of these considerations, there is much effort being invested into developing radiomitigating compounds specifically for HEM-ARS.

In our quest to develop a radiomitigating compound for use in HEM-ARS we first had to identify a lead compound and demonstrate efficacy in our primary endpoint (Specific Aim 1). Quinic acid derivative KZ-41 was identified as our compound of interest and we tested its efficacy in a murine lethal total body irradiation 30 day mortality study. KZ-41 significantly improved survival when administered 25 hours after lethal $\left(\mathrm{LD}_{80 / 30}\right)$ total body irradiation. Armed with this information, we sought to learn more about the efficacy of KZ-41, particularly in the setting of preventing radiationinduced abnormalities in thrombus formation.

There are few established animal models to study the effects of ARS eliciting doses of radiation and potential mitigation by a compound of interest. Therefore, we developed a live animal imaging model to study the effects of vascular injury in the setting of high dose TBI (HEM-ARS doses). We identified our primary endpoint as thrombus formation, and optimized the time after irradiation to maximize the difference in thrombus formation. We correlated thrombus formation with circulating platelet numbers as a secondary endpoint. We then tested the radiomitigating (12 hours after TBI) efficacy of KZ-41 on radiation-induced abnormalities in thrombus formation. KZ41 significantly increased the probability of thrombus formation after irradiation without affecting the circulating platelet numbers. With this second piece of efficacy data, we focused our aim on the mechanism of action of KZ-41.

To investigate the mechanism of action of the radiomitigating effects of KZ-41 (Specific Aim 2) we chose to develop an in vitro model in human U937 cells. We chose this monocyte-like cell line because of published reports concluding that death from HEM-ARS requires mitochondrial dependent apoptosis in hematopoietic cells. Because we were developing radiomitigating compounds, we believed that we required a model that allowed us to investigate indirect or bystander effects. We demonstrated that a bystander effect can be elicited in the U937 cell line through soluble factors by a series of conditioned medium transfer experiments, and that KZ-41 dose-dependently reduces 
Caspse-3/7 activation which is partly due to inhibition of Caspase-8. Although we are making progress towards the mechanism of action of KZ-41, further studies are required.

In an effort to improve upon our existing lead compound, KZ-41, we were interested in incorporating the proven efficacy of antioxidants in radiation injury (Specific Aim 3). Our goal was to retain the active portion of chlorogenic acid (caffeic acid) and to substitute a known biologically active molecule for the quinic acid portion. We substituted KZ-41 for the QA portion of the molecule because of its proven efficacy in our desired setting, Acute Radiation Syndrome. The result was KZ-157 a next generation KZ-41 molecule and chlorogenic acid derivative. We investigated the initial pharmacokinetic parameters of KZ-157 both in vivo and in vitro. We first developed an LC-MS/MS method that enabled us to quantify KZ-157 and its primary metabolites in biological matrices. We then administered KZ-157 to rats both intravenously and orally to characterize the pharmacokinetics, and evaluated plasma protein binding and stability in the presence of liver microsomes. KZ-157 demonstrates two-compartment pharmacokinetics with a rapid absorption and a low bioavailability following oral administration and is rapidly eliminated.

In summary, we have identified and demonstrated radiomitigating efficacy of novel radiomitigant KZ-41 in multiple animal models. We are beginning to understand the mechanism of action of its radiomitigating effects via the in vitro assay that we developed. The in vitro model that we have developed may also be used to screen for next-generation radiomitigating compounds. 


\section{LIST OF REFERENCES}

1. Weisdorf, D., et al., Acute radiation injury: contingency planning for triage, supportive care, and transplantation. Biology of Blood and Marrow Transplantation, 2006. 12(6): p. 672-682.

2. Koenig, K.L., et al., Medical treatment of radiological casualties: current concepts. Annals of Emergency Medicine, 2005. 45(6): p. 643-652.

3. Stone, H.B., et al., Models for evaluating agents intended for the prophylaxis, mitigation and treatment of radiation injuries: report of an NCI Workshop, December 3-4, 2003. Radiat Res, 2004. 162(6): p. 711-728.

4. Mettler, F.A., Jr., A.K. Gus'kova, and I. Gusev, Health effects in those with acute radiation sickness from the Chernobyl accident. Health Phys, 2007. 93(5): p. 462469.

5. Waselenko, J.K., et al., Medical management of the acute radiation syndrome: recommendations of the Strategic National Stockpile Radiation Working Group. Ann Intern Med, 2004. 140(12): p. 1037-1051.

6. Coleman, C.N., et al., Molecular and cellular biology of moderate-dose (1-10 Gy) radiation and potential mechanisms of radiation protection: report of a workshop at Bethesda, Maryland, December 17-18, 2001. Radiat Res, 2003. 159(6): p. 812834.

7. $\quad$ Ojielo, C.I., et al., Defective phagocytosis and clearance of Pseudomonas aeruginosa in the lung following bone marrow transplantation. J Immunol, 2003. 171(8): p. 4416-4424.

8. $\quad$ Fliedner, T.M., H.D. Dorr, and V. Meineke, Multi-organ involvement as a pathogenetic principle of the radiation syndromes: a study involving 110 case histories documented in SEARCH and classified as the bases of haematopoietic indicators of effect. Br J Radiol, 2005. 27(Supplement 1): p. 1-8.

9. Fliedner, T.M., Nuclear terrorism: the role of hematology in coping with its health consequences. Current Opinion in Hematology, 2006. 13(6): p. 436-444.

10. Srinivasan, V., et al., Evaluation and development of radiation countermeasures at AFRRI. AFRRI Report 2005.

11. McDonough, J.H., P.C. Mele, and C.G. Franz, Comparison of behavioral and radioprotective effects of WR-2721 and WR-3689. Pharmacol Biochem Behav, 1992. 42(2): p. 233-243.

12. Lee, H.J., et al., Amifostine ameliorates recognition memory defect in acute radiation syndrome caused by relatively low-dose of gamma radiation. J Vet Sci. 11(1): p. 81-83.

13. Stickney, D.R., et al., 5-androstenediol stimulates multilineage hematopoiesis in rhesus monkeys with radiation-induced myelosuppression. Int Immunopharmacol, 2006. 6(11): p. 1706-1713.

14. Blakely, W.F., et al., Amylase and blood cell-count hematological radiationinjury biomarkers in a rhesus monkey radiation model--use of multiparameter and integrated biological dosimetry. Radiation Measurements, 2007. 42(6-7): p. 1164-1170. 
15. Biomolecular Action of Ionizing Radiation, ed. S. Lehnert. 2008: New York, Taylor \& Francis. 527.

16. Elmore, S., Apoptosis: a review of programmed cell death. Toxicol Pathol, 2007. 35(4): p. 495-516.

17. Kirsch, D.G., et al., p53 controls radiation-induced gastrointestinal syndrome in mice independent of apoptosis. Science, 2010. 327(5965): p. 593-596.

18. Bertho, J.M. and L. Roy, A rapid multiparametric method for victim triage in cases of accidental protracted irradiation or delayed analysis. Br J Radiol, 2009. 82(981): p. 764-770.

19. Prat, M., et al., Use of flt3 ligand to evaluate residual hematopoiesis after heterogeneous irradiation in mice. Radiat Res, 2006. 166(3): p. 504-511.

20. Ballinger, M.N., et al., Paradoxical role of alveolar macrophage-derived granulocyte-macrophage colony-stimulating factor in pulmonary host defense post-bone marrow transplantation. Am J Physiol Lung Cell Mol Physiol, 2008. 295(1): p. L114-122.

21. Baranov, A.E., et al., Hematopoietic recovery after 10-Gy acute total body radiation. Blood, 1994. 83(2): p. 596-599.

22. Trivedi, M., et al., Optimal use of G-CSF administration after hematopoietic SCT. Bone Marrow Transplant, 2009. 43: p. 895-908.

23. Fliedner, T.M., et al., Pathophysiological principles underlying the blood cell concentration responses used to assess the severity of effect after accidental whole-body radiation exposure: an essential basis for an evidence-based clinical triage. Experimental Hematology, 2007. 35(4, Supplement 1): p. 8-16.

24. Guipaud, O. and M. Benderitter, Protein biomarkers for radiation exposure: towards a proteomic approach as a new investigation tool. Ann Ist Super Sanita, 2009. 45(3): p. 278-286.

25. Becciolini, A., et al., Plasma amylase activity as a biochemical indicator of radiation injury to salivary glands. Acta Radiol Oncol, 1984. 23(1): p. 9-14.

26. Fichelson, S., The FLT3/FLK2 ligand: structure, functions and prospects. Eur Cytokine Netw, 1998. 9(1): p. 7-22.

27. Bertho, J.M., et al., New Biological indicators to evaluate and monitor radiationinduced damage: an accident case report. Radiation Research, 2009. 169(5): p. 543-550.

28. Mettler, F.A., Jr. and G.L. Voelz, Major radiation exposure--what to expect and how to respond. N Engl J Med, 2002. 346(20): p. 1554-1561.

29. Dainiak, N., et al., The hematologist and radiation casualties. Hematology Am Soc Hematol Educ Program, 2003: p. 473-946.

30. Sorensen, D.K., et al., An effective therapeutic regimen for the hemopoietic phase of the acute radiation syndrome in dogs. Radiation Research, 1960. 13: p. 669685.

31. Fakir, H., et al., Triggering-response model for radiation-induced bystander effects. Radiat Res, 2009. 171(3): p. 320-331.

32. Herok, R., et al., Bystander effects induced by medium from irradiated cells: similar transcriptome responses in irradiated and bystander K562 cells. International Journal of Radiation Oncology*Biology*Physics. 77(1): p. 244-252.

33. Hall, E.J., The bystander effect. Health Phys, 2003. 85(1): p. 31-35. 
34. Kashino, G., et al., Radiation induced bystander signals are independent of DNA damage and DNA repair capacity of the irradiated cells. Mutat Res, 2007. 619(12): p. 134-138.

35. Guicciardi, M.E. and G.J. Gores, Life and death by death receptors. FASEB J., 2009. 23(6): p. 1625-1637.

36. Kim, E.M., et al., Amplification of the gamma-irradiation-induced cell death pathway by reactive oxygen species in human U937 cells. Cell Signal, 2008. 20(5): p. 916-924.

37. Schoene, N.W., et al., Water-soluble polymeric polyphenols from cinnamon inhibit proliferation and alter cell cycle distribution patterns of hematologic tumor cell lines. Cancer Lett, 2005. 230(1): p. 134-140.

38. Pawlik, T.M. and K. Keyomarsi, Role of cell cycle in mediating sensitivity to radiotherapy. Int J Radiat Oncol Biol Phys, 2004. 59(4): p. 928-942.

39. Farah, A., et al., Chlorogenic acids from green coffee extract are highly bioavailable in humans. J Nutr, 2008. 138(12): p. 2309-2315.

40. Gonthier, M.P., et al., Chlorogenic acid bioavailability largely depends on its metabolism by the gut microflora in rats. J. Nutr., 2003. 133(6): p. 1853-1859.

41. Scalbert, A. and G. Williamson, Dietary intake and bioavailability of polyphenols. J. Nutr., 2000. 130(8): p. 2073S-2085.

42. Suzuki, A., et al., Green coffee bean extract and its metabolites have a hypotensive effect in spontaneously hypertensive rats. Hypertens Res, 2002. 25(1): p. 99-107.

43. Rechner, A.R., et al., The metabolic fate of dietary polyphenols in humans. Free Radic Biol Med, 2002. 33(2): p. 220-235.

44. Zhang, J., et al., Liquid chromatograph/tandem mass spectrometry assay for the simultaneous determination of chlorogenic acid and cinnamic acid in plasma and its application to a pharmacokinetic study. J Pharm Biomed Anal, 2010. 51(3): p. 685-690.

45. Yang, H., et al., HPLC determination and pharmacokinetics of chlorogenic acid in rabbit plasma after an oral dose of Flos Lonicerae extract. J Chromatogr Sci, 2004. 42(4): p. 173-176.

46. Ren, J., X. Jiang, and C. Li, Investigation on the absorption kinetics of chlorogenic acid in rats by HPLC. Arch Pharm Res, 2007. 30(7): p. 911-916.

47. Ren, J., et al., Absorptive profile of chlorogenic acid in rats. Pharmazie, 2007. 62(9): p. 689-692. 


\section{VITA}

Karin Emmons Thompson was born in Jonesboro, Arkansas, United States of America, in 1983. She began her undergraduate studies in 2001 at Arkansas State University in Jonesboro, Arkansas, majoring in Biological Sciences and received a Bachelor of Science in 2004. In 2005, she joined the University of Tennessee Health Science Center, Memphis, Tennessee. She received the Doctor of Philosophy degree in Pharmaceutical Sciences under the supervision of Professor Charles R. Yates in 2010. 